\title{
An individualized multimodal mental skills intervention for college athletes undergoing injury rehabilitation
}

Jamie L. Shapiro

West Virginia University

Follow this and additional works at: https://researchrepository.wvu.edu/etd

\section{Recommended Citation}

Shapiro, Jamie L., "An individualized multimodal mental skills intervention for college athletes undergoing injury rehabilitation" (2009). Graduate Theses, Dissertations, and Problem Reports. 4530.

https://researchrepository.wvu.edu/etd/4530

This Dissertation is protected by copyright and/or related rights. It has been brought to you by the The Research Repository @ WVU with permission from the rights-holder(s). You are free to use this Dissertation in any way that is permitted by the copyright and related rights legislation that applies to your use. For other uses you must obtain permission from the rights-holder(s) directly, unless additional rights are indicated by a Creative Commons license in the record and/ or on the work itself. This Dissertation has been accepted for inclusion in WVU Graduate Theses, Dissertations, and Problem Reports collection by an authorized administrator of The Research Repository @ WVU.

For more information, please contact researchrepository@mail.wvu.edu. 


\title{
An Individualized Multimodal Mental Skills Intervention for College Athletes Undergoing Injury Rehabilitation
}

\author{
Jamie L. Shapiro \\ Dissertation submitted to \\ the College of Physical Activity and Sport Sciences \\ at West Virginia University \\ in partial fulfillment of the requirements for the degree of
}

Doctor of Philosophy

in Kinesiology with a major in Sport and Exercise Psychology

\author{
Edward Etzel, Ed.D., Chair \\ Andrew Hawkins, Ph.D. \\ Allison Hetrick, M.Ed., ATC, CSCS \\ Ed Jacobs, Ph.D. \\ Samuel Zizzi, Ed.D.
}

Morgantown, West Virginia

2009

Keywords: Sport Injury; Psychology; Goal Setting; Relaxation; Imagery; Self-talk 


\begin{abstract}
An Individualized Multimodal Mental Skills Intervention for College Athletes Undergoing Injury Rehabilitation
\end{abstract}

Jamie L. Shapiro

Since the response to injury is both physical and psychological (e.g., Brewer \& Cornelius, 2003; Wiese-Bjornstal, Smith, Shaffer, \& Morrey, 1998), numerous researchers have advocated using psychological interventions in conjunction with physical therapy to optimize an athlete's recovery from injury. No empirical study has examined how to craft a mental skills intervention for injured athletes from initial post-injury assessment. The purpose of the present study was to explore injured athletes' experiences with a multimodal mental skills intervention to gain insight into how to create a mental skills plan for rehabilitation from initial post-injury assessment. Single subject and qualitative methodology were utilized. Six participants completed the approximately eight week study in which participants learned four mental skills: (1) goal setting; (2) relaxation; (3) imagery; and (4) managing self-talk. Using an ABCA or ACBA design, the impact of the interventions on the following eight variables was examined: (1) self-reported use of mental skills; (2) perceived effectiveness of mental skills; (3) satisfaction with mental skills interventions; (4) self-efficacy for rehabilitation; (5) self-efficacy for return to sport; (6) adherence; (7) attitude; and (8) speed of recovery. Participants showed varied responses to the mental skills interventions and reported several positive physical and psychological outcomes of using the mental skills. Participants demonstrated little change in self-efficacy for rehabilitation and return to sport, adherence, attitude, and speed of recovery over the course of the study, as most participants scored high throughout the study on these measures. Sport psychology consultants and future researchers may want to explore other post-injury assessment methods and choose less than four mental skills to focus on for a mental rehabilitation program with injured athletes. Athletic trainers can also incorporate simple mental techniques in rehabilitation to help athletes achieve positive outcomes. 


\section{ACKNOWLEDGEMENTS}

Dr. Ed: I am enormously grateful to you for acting as my chair and getting me through this final push to the end! Your kindness, patience, guidance, and speedy feedback were extremely appreciated! I definitely owe you some NJ bagels for this!

Dr. Zizzi: Thank you for serving as a mentor and advisor, and for getting me through the dissertation proposal. You may not have broken my procrastination habit, but alas, here is a document. It may have taken a few all-nighters, but...

Kim Crowell and Dr. Shannon: Thanks for all your help with the qualitative analyses and coding. Remember, "if you're about to die, you definitely positive self-talk.” Kim: Thanks for all your hard work as my "research assistant” that I volunteered you for! I really appreciate your help.

Dr. Hawkins: Thank you for helping me with interpreting the (way too many) graphs in my study!

Dr. Jacobs and Allison Hetrick: Thank you for serving on my dissertation committee. Dr. Jacobs: thanks also for being a great teacher and mentor for me in counseling.

Kevin Kotsko, Jimmy Dorneman, and Regina Hash: I can't thank you enough for helping me recruit participants and completing the assessments for the study. This dissertation could not have happened without your help - thanks for helping me become "Dr. Shapiro."

Participants: I want to express my sincere gratitude to the participants in my study for taking time out of their busy schedules and volunteering to complete my study. Without you, there would be no dissertation!

All my teachers and professors: Thank you for sharing your knowledge and wisdom that helped me get to this point in my career and shaped me into the professional I am today.

Colleagues and friends in the SEP program: I cannot believe I'm at the end the road here at WVU - thanks for helping me get there and making my time in Morgantown so memorable! Your friendship, encouragement, and support during my time here have meant more to me than you know. I wish nothing but the best for each and every one of you! A special thanks to Michelle, Katie, and Sean for always being there when I needed to vent!

My parents: Words cannot express how much I appreciate your support in allowing me to pursue my "dream" career path, even though you may not understand it at times! I could not have gotten to this point in my life without you. Brian: thanks to you also for your support. It's been fun to watch you grow up into a young man! Love you guys with all my heart!

Finally, thank you to the Office of Graduate Education at WVU for providing me with the Dissertation Fellowship so that I may focus on this project and complete it this semester. 


\section{TABLE OF CONTENTS}

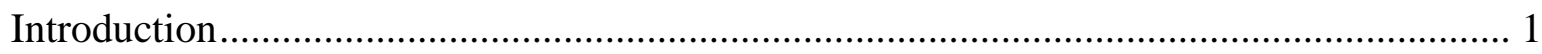

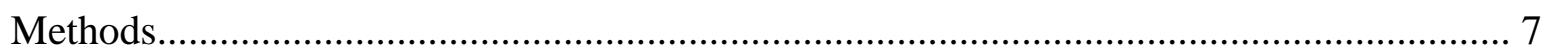

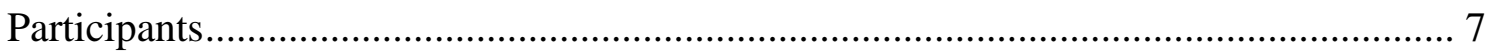

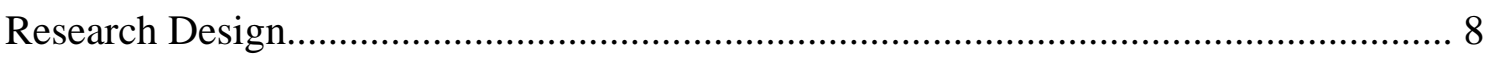

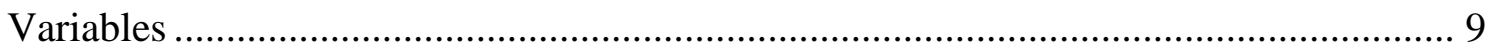

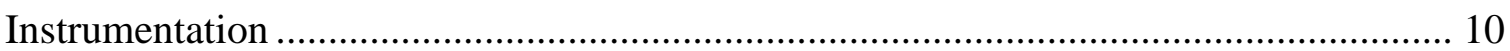

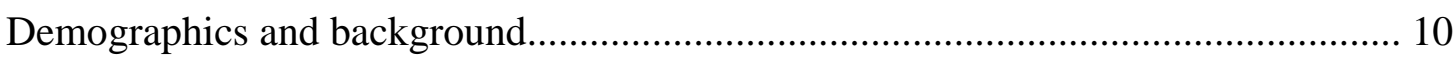

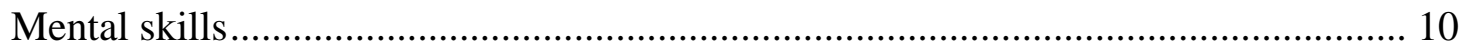

Monitoring of mental skills intervention ....................................................... 12

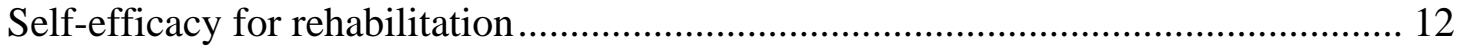

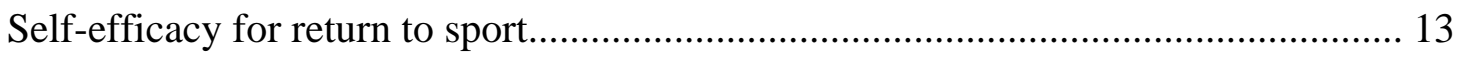

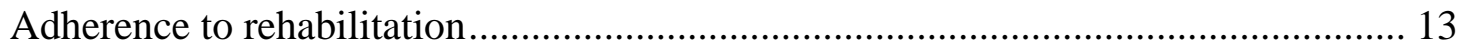

Recovery time and attitude towards rehabilitation ............................................. 14

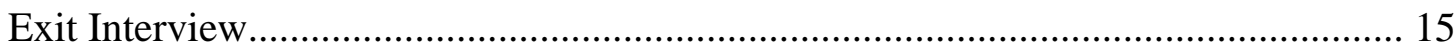

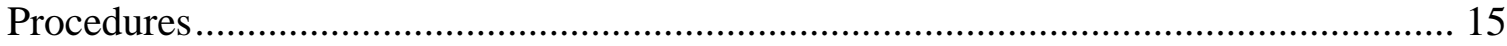

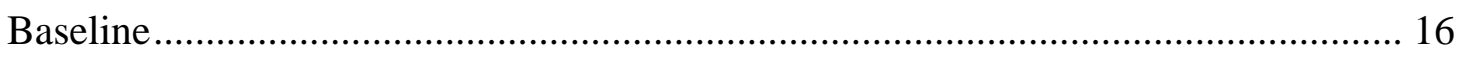

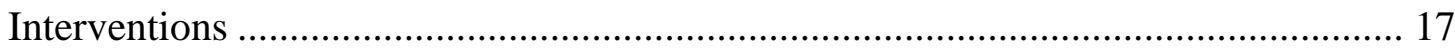

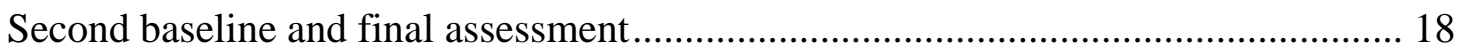

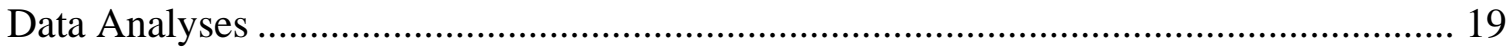

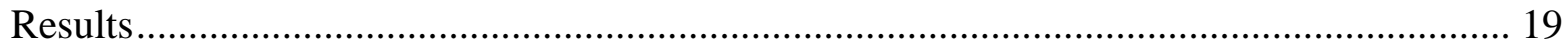

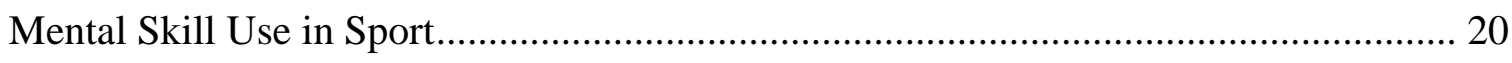

Self-reported Use of Mental Skills in Rehabilitation................................................. 20 
Perceived Effectiveness of Mental Skills ........................................................................ 25

Satisfaction with Mental Skills Interventions ............................................................ 26

Additional Qualitative Data Regarding Mental Skills Interventions................................. 28

Self-efficacy for Rehabilitation................................................................................ 29

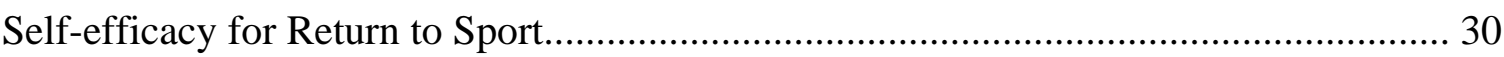

Adherence to the Rehabilitation Protocol ............................................................................ 30

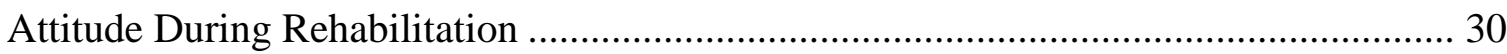

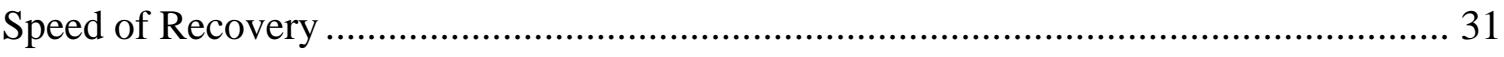

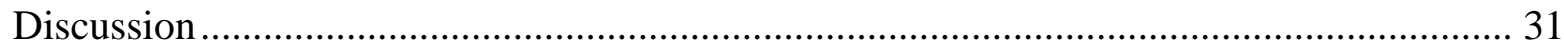

Assessment of Mental Skill Strengths and Weaknesses ................................................... 31

Self-reported Mental Skill Use Throughout the Study ...................................................... 32

Perceived Effectiveness of the Interventions ...................................................................... 35

Satisfaction with Mental Skills Interventions and Future Use of Skills ........................... 36

Self-efficacy, Adherence, Attitude, and Speed of Recovery ………................................ 38

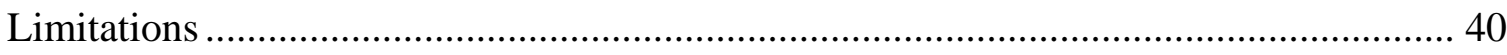

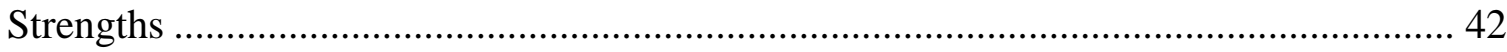

Practical Implications and Future Directions.................................................................. 43

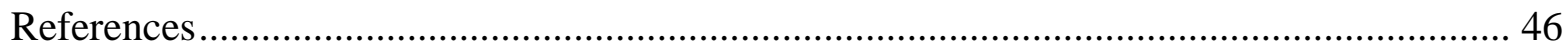

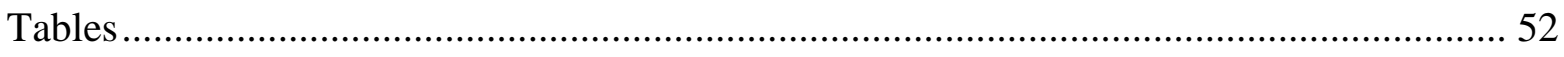

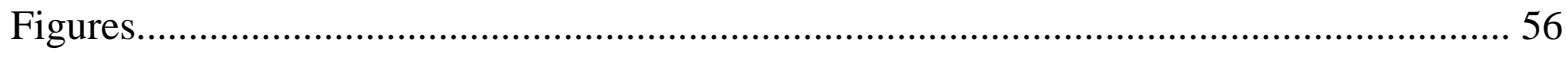

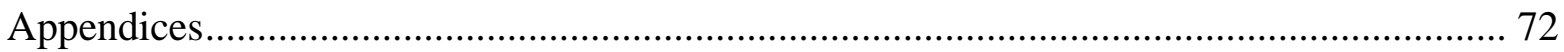

APPENDIX A. Review of the Literature..................................................................... 72

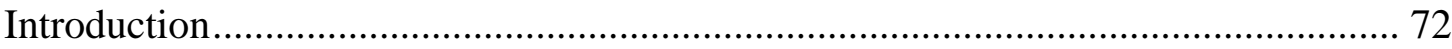


Models of Psychological Responses to Sport Injury 72

Psychological Factors Associated with Injury Rehabilitation Outcomes ..................... 76

Suggestions for Implementing Psychological Interventions for Sport Injury

Rehabilitation 83

Empirical Research on Psychological Interventions for Injury Rehabilitation 86

Summary 102

References 104

APPENDIX B. Initial Interview 111

APPENDIX C. Test of Performance Strategies-2 …………………………………….... 112

APPENDIX D. Monitoring of Mental Skills Intervention ............................................... 116

APPENDIX E. Athletic Injury Self-Efficacy Questionnaire.............................................. 117

APPENDIX F. Modified State Sport Confidence Inventory ............................................... 118

APPENDIX G. Athletic Trainer's Patient Evaluation Form .............................................. 119

APPENDIX H. Athlete-rated Adherence and Attitude Items........................................... 120

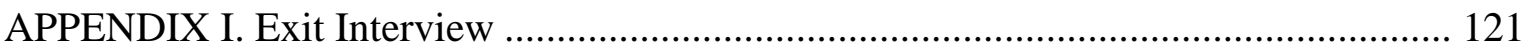

APPENDIX J. Pilot Research ..................................................................................... 123

APPENDIX K. Approval from Institutional Review Board.............................................. 130

APPENDIX L. Informed Consent Form for Athletes........................................................ 131

APPENDIX M. Informed Consent Form for Athletic Trainers.......................................... 136

APPENDIX N. Self-ranking of Mental Skills Use.......................................................... 140

APPENDIX O. Sample Outlines of Mental Skills Interventions ....................................... 141

APPENDIX P. Sample Intervention Materials ................................................................. 145

APPENDIX Q. Participant Graphs for: Self-efficacy for Return to Sport, Adherence, Attitude, Recovery Time......................................................................... 170 


\section{LIST OF TABLES}

Table 1. Demographic Information of Participants ....................................................... 52

Table 2. Test of Performance Strategies (TOPS-2) Data.................................................. 54

Table 3. Satisfaction Ratings of Each Mental Skills Intervention .................................... 55 


\section{LIST OF FIGURES}

Figure 1a. Self-reported use of mental skills (strengths) for P1 .................................... 56

Figure 1b. Perceived effectiveness of mental skills (strengths) on rehabilitation for P1 ...... 56

Figure 2a. Self-reported use of mental skills (weaknesses) for P1 ................................. 57

Figure 2b. Perceived effectiveness of mental skills (weaknesses) on rehabilitation for P1 .. 57

Figure 3a. Self-reported use of mental skills (strengths) for P2 ................................... 58

Figure 3b. Perceived effectiveness of mental skills (strengths) on rehabilitation for P2 ..... 58

Figure 4a. Self-reported use of mental skills (weaknesses) for P2 ................................ 59

Figure 4b. Perceived effectiveness of mental skills (weaknesses) on rehabilitation for P2 .. 59

Figure 5a. Self-reported use of mental skills (strengths) for P3 ..................................... 60

Figure 5b. Perceived effectiveness of mental skills (strengths) on rehabilitation for P3 ...... 60

Figure 6a. Self-reported use of mental skills (weaknesses) for P3 ................................ 61

Figure 6b. Perceived effectiveness of mental skills (weaknesses) on rehabilitation for P3 .. 61

Figure 7a. Self-reported use of mental skills (strengths) for P4 ................................... 62

Figure 7b. Perceived effectiveness of mental skills (strengths) on rehabilitation for P4 ..... 62

Figure 8a. Self-reported use of mental skills (weaknesses) for P4 ................................ 63

Figure 8b. Perceived effectiveness of mental skills (weaknesses) on rehabilitation for P4 .. 63

Figure 9a. Self-reported use of mental skills (strengths) for P5 ................................... 64

Figure 9b. Perceived effectiveness of mental skills (strengths) on rehabilitation for P5 ...... 64

Figure 10a. Self-reported use of mental skills (weaknesses) for P5 ................................ 65

Figure 10b. Perceived effectiveness of mental skills (weaknesses) on rehabilitation

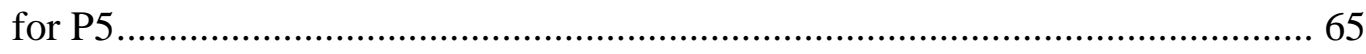

Figure 11a. Self-reported use of mental skills (strengths) for P6 ................................... 66

Figure 11b. Perceived effectiveness of mental skills (strengths) on rehabilitation for P6 .... 66 
Figure 12a. Self-reported use of mental skills (weaknesses) for P6.

Figure 12b. Perceived effectiveness of mental skills (weaknesses) on rehabilitation

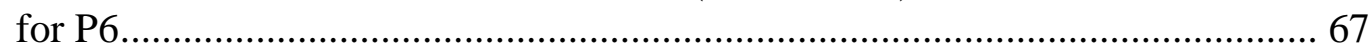

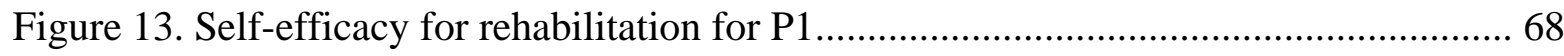

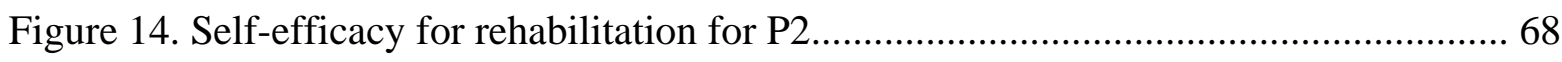

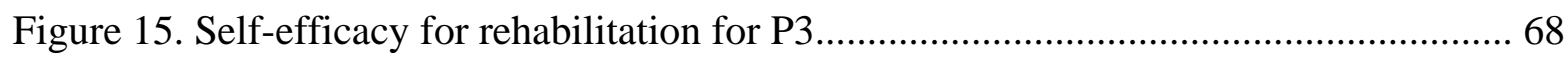

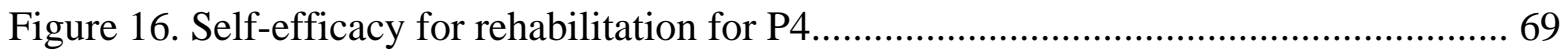

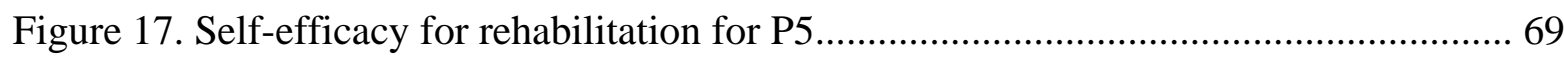

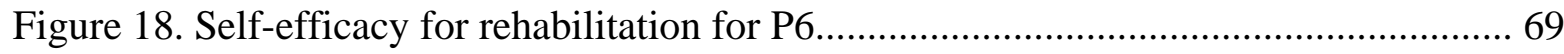

Figure 19. Self-rated adherence and attitude for P1 …..................................................... 70

Figure 20. Athletic Trainer-rated data for P3..................................................................... 70

Figure 21. Self-rated adherence and attitude for P3 ………................................................ 70

Figure 22. Athletic Trainer-rated data for P1................................................................... 71

Figure 23. Self-rated adherence and attitude for P6 …........................................................ 71 


\section{Introduction}

It is estimated that between three and 17 million children and adults incur sport and recreation injuries each year in the United States (Brewer, Anderson, \& Van Raalte, 2002). Recovery from sport injury includes both physical and psychological factors. Wiese-Bjornstal, Smith, Shaffer, and Morrey (1998) proposed an integrated model of response to sport injury and rehabilitation. They maintain that personal, situational, and cognitive factors interact to influence one’s holistic responses to athletic injury. Accordingly, an athlete post-injury makes a cognitive appraisal of the injury and his/her self-efficacy, or belief in ability, to cope with the situation. The cognitive appraisals, emotional responses (e.g., mood, attitude, fear, grief), and behavioral responses (e.g., rehabilitation adherence, effort and intensity in rehabilitation, use of psychological skills) are believed to reciprocally influence each other throughout the recovery process.

Taking into account the complexity of the response to injury, injured athletes will likely benefit from a rehabilitation protocol that incorporates both physical and psychological interventions. Several authors (e.g., Crossman, 1997, 2001; O’Conner, Heil, Harmer, \& Zimmerman, 2005; Petitpas \& Danish, 1995; Taylor \& Taylor, 1997) have advocated the use of psychological interventions and have proposed methods for implementation. For example, Taylor and Taylor (1997) suggest assessing an athlete’s psychological needs prior to intervention and then tailoring the mental skills program to the athlete's needs and preferences. Specifically, the authors encourage athletes to select one or two techniques per psychological need based on “comfort and liking” (p. 299). They specifically suggest that rehabilitation imagery be part of every program because of its generalized usefulness to recovery. Some interventions that the 
authors recommend including in the mental skills program include goal setting, relaxation, imagery, self-talk, social support, and confidence building.

Although Taylor and Taylor (1997) based some of their recommendations on the literature, much of their practical advice was based on their experiences working with athletes. While this information is valuable for practitioners, it is also essential to examine what the literature says about psychological interventions with injured athletes. Some studies have been conducted that look at athletes' self-reported use of psychological strategies and how these relate to rehabilitation outcomes. For example, Ievleva and Orlick (1991) found that fast healers retrospectively reported using more goal setting, positive self-talk, and imagery than slow healers; goal setting emerged as the most important healing variable. In another study, Scherzer et al. (2001) found that goal setting and positive self-talk were related to adherence measures.

In another qualitative investigation, Driedger, Hall, and Callow (2006) found that rehabilitating athletes reported using imagery for a variety of purposes including: to help maintain sport skills, motivation, focus, healing, pain management, and future injury prevention. Milne, Hall, and Forwell (2005) found that mental rehearsal of rehabilitation exercises was a significant predictor of task self-efficacy for rehabilitation. Those researchers also found that task and coping self-efficacy were predictive of better rehabilitation adherence. Thus, practitioners should work to build athletes' self-efficacy during the rehabilitation process to maximize outcomes. This lends support to self-efficacy theory (Bandura, 1977), which posits that the belief in one's ability to perform a task is important for the accomplishment of behavioral outcomes.

A major problem with the above studies is that they relied on retrospective recall of strategies reportedly used during recovery. Therefore, studies of psychological interventions, 
such as those where athletes learn mental skills (e.g., goal setting, relaxation, imagery, and managing self-talk), can help guarantee that athletes are using the skills.

Psychological interventions for injured athletes have been perceived by athletes and athletic trainers (ATCs) to be at least moderately acceptable to use during injury rehabilitation (Brewer, Jeffers, Petitpas, \& Van Raalte, 1994; Francis, Andersen, \& Maley, 2000; Myers, Peyton, and Jensen, 2004; Ninedek \& Kolt, 2000; Wiese, Weiss, \& Yukelson, 1991).

Researchers have found a preference for goal setting and positive self-talk over relaxation and imagery when exploring perceptions of injured athletes and physical therapists (Brewer et al.; Francis et al.; Ninedek \& Kolt; Wiese et al.).

Despite support that psychological techniques can benefit recovery, empirical intervention studies with injured athletes are somewhat scarce (Cupal, 1998). Intervention studies with injured athletes have been conducted for the following psychological techniques: goal setting, relaxation, imagery, cognitive restructuring, stress management, counseling, and combinations of these skills (multimodal interventions).

Relative to goal setting, researchers have found that some injured athletes who set goals showed improved outcomes compared to control groups that did not set goals. Some of these outcomes included better performance on rehabilitation tasks (Theodorakis, Beneca, Malliou, \& Goudas, 1997; Theodorakis, Malliou, Papioannou, Beneca, \& Filactakidou, 1996), improved adherence to the rehabilitation program, and higher self-efficacy to complete the rehabilitation program (Evans \& Hardy, 2002a). A qualitative follow-up study by Evans and Hardy (2002b) highlighted the importance of individual difference variables in response to the intervention, including goal type preference (long-term, short-term, outcome, or performance goals). 
Relaxation combined with guided imagery is another intervention that has been examined with injured athletes. With a sample of participants who had knee surgery, Cupal and Brewer (2001) found that those who practiced relaxation/imagery sessions for six months following surgery had significantly greater knee strength, less reinjury anxiety, and less pain than participants in the control and placebo groups. Handegard, Joyner, Burke, and Reimann (2006) explored the effects of relaxation combined with healing imagery with two injured male athletes. Qualitative statements indicated that the participants felt that the imagery helped give them a sense of control in their recovery and feel more mentally prepared to return to sport.

Several other studies have evaluated multimodal psychological interventions with injured athletes. Johnson (2000) explored the effectiveness of a short-term psychological intervention for a sample of athletes with long-term injuries. The participants in the experimental group attended three different training sessions including: (1) stress management and cognitive control, (2) goal setting, and (3) relaxation/guided imagery. At the midpoint and at the end of rehabilitation, the experimental group had a higher overall mood state than did the control group. Ross and Berger (1996) investigated the efficacy of a cognitive-behavioral intervention, specifically stress inoculation training (SIT), on injured athletes’ postsurgical anxiety, pain, and physical functioning. This study was also multimodal in that athletes in the experimental group learned several skills as part of the stress inoculation training, including relaxation, imagery, and managing self-talk. Those researchers found that both state anxiety and pain decreased over time but more rapidly for participants in the treatment condition than the control condition. They also discovered that the treatment group reported significantly less anxiety and less pain than the control group. Finally, the researchers found that the mean number of days to recovery for the 
treatment group was significantly less than the mean number of recovery days for the control group.

Evans, Hardy, and Fleming (2000) explored the efficacy of a multimodal intervention involving consultations with three injured athletes, using techniques such as goal setting, imagery, social support, verbal persuasion, and simulation training (i.e., executing skills during practice that were of concern due to the injury). Qualitative analyses indicated the usefulness of certain techniques during certain phases of rehabilitation. For example, participants implemented goal setting to improve motivation and adherence during the early and middle phases of rehabilitation, while it was used to build self-efficacy during the later phase of recovery.

In a single subject study exploring relaxation and cognitive interventions with injured athletes, Naoi and Ostrow (2008) found inconsistent results among participants, with individual differences in mood and pain outcomes in response to the interventions. Participants perceived the cognitive intervention to be more effective on physical and psychological recovery than the relaxation intervention. Naoi and Ostrow’s study could have benefited from qualitative analyses to enhance the interpretability of the findings and learn more about participants’ experiences with the interventions.

In another single subject study, Rock and Jones (2002) used both quantitative and qualitative measures to explore the impact of six counseling sessions over 12 weeks post-surgery for three injured athletes. By the end of the rehabilitation period monitored, mood disturbance had fallen below pre-surgery levels for all participants. Also, perceptions of rehabilitation progress increased while pain ratings decreased, although both fluctuated, particularly in response to setbacks. A concern with these findings is that it is difficult to determine if the above variables (mood, perceived progress, and pain) changed due to time or the intervention. 
Interview responses indicated that two of the three participants had favorable impressions of the intervention. The researchers also found that the intervention reduced the negative impact of setbacks on mood and perceptions of rehabilitation progress. Finally, the researchers highlighted the importance of considering individual differences during counseling, as each participant's experience was unique.

In many of the above intervention studies, the match between the intervention and the participant was not included in the design. In other words, the intervention was not chosen based on the athlete's needs or preferences, but rather chosen by the researcher(s). Although two of the studies (Evans et al., 2000; Rock \& Jones, 2002) used more flexible and individualized psychological interventions, none of these studies included a needs assessment before implementing the intervention, as Taylor and Taylor (1997) suggested. In addition, several studies highlighted the differences in individual responses to the psychological interventions (Evans \& Hardy, 2002b; Evans et al., 2000; Naoi \& Ostrow, 2008; Rock \& Jones, 2002). However, few studies have explored these individual responses.

The findings in the majority of psychological intervention studies provide some support for the positive benefits of such interventions for injured athletes, but what works best? How should practitioners working with injured athletes (e.g., sport psychology consultants, sports medicine professionals, counselors) craft a psychological skills intervention to best meet the needs of the injured athlete? Weinberg and Gould (2007) stated, “the most important psychological skills to learn for rehabilitation are goal setting, positive self-talk, imagery visualization, and relaxation training” (p. 457). How many of these skills should be chosen for a mental skills program for injured athletes? Should the skills be chosen based on "comfort and liking” as Taylor and Taylor (1997) suggested? “Comfort and liking” seems vague, and appears 
to suggest that skills should be chosen that the athlete is familiar with; however, one might argue that an athlete may benefit more from learning how to apply a new skill to injury recovery and to sport.

The purpose of the present study was to examine individual injured athletes’ experiences with a personalized multimodal mental skills intervention in order to gain insight into how to create such an intervention from initial post-injury assessment. Both single subject and qualitative methodologies were used. It was hypothesized that the following dependent variables would improve during the intervention phases of the study: perceived effectiveness of mental skills, self-efficacy for rehabilitation, self-efficacy for return to sport, adherence, attitude in rehabilitation, and speed of recovery. Further, it was hypothesized that scores would be higher during the intervention phase for the mental skills that were identified as an athlete's strengths (B phase) versus weaknesses ( $\mathrm{C}$ phase). The same would be true for satisfaction ratings of the mental skills. For the dependent variable of self-reported use of mental skills, it was hypothesized that the use of all skills would increase during the intervention phase in which the skill was taught. Also, reported use of the skills identified as strengths would respond better to the intervention than those identified as weaknesses. Finally, all dependent variables measured would be higher during the second baseline than the first baseline, but they would not be higher than during the intervention phases.

\section{Methods}

\section{Participants}

Participants who completed the study were five male athletes from an NCAA Division I University and one female athlete from an NCAA Division III University. Volunteer participants were recruited from the athletic training rooms of the two universities. A purposive sampling 
method was used. The five inclusion criteria were: (1) the athletes incurred acute injuries that required a rehabilitation period of at least two months; (2) the injury occurred during a sport activity; (3) the injury required an active rehabilitation (i.e., no cast where they were immobile or needed to rest only); (4) the injury was not career ending; and (5) the athlete had not experienced a re-injury. Athletes began participating in the study between 2 and 6 weeks post-surgery $(M=$ 3.67, $S D=1.63)$.

The mean age of participants was $19.3(S D=1.4)$. They reported that they were involved in the sports of wrestling $(n=3)$, soccer $(n=2)$, and cheerleading $(n=1)$. Participants all incurred injuries that required surgery, including surgery of the knee $(n=3)$, shoulder $(n=2)$, and ankle $(n=1)$. Participants identified themselves as Caucasian $(n=5)$ and Mexican American $(n=1)$. Additional participant information can be found in Table 1. Three athletic trainers (ATCs) who worked with the athletes in the study were also participants, as they completed assessments to evaluate the athletes' adherence and progress. Two of the athletic trainers (male) worked at the Division I institution, while the other athletic trainer (female) worked at the Division III institution.

\section{Research Design}

The study used mixed methods, incorporating both quantitative and qualitative methodology. The quantitative aspect was a single-subject design, either ABCA or ACBA in nature. The "A" phase was baseline, the " $\mathrm{B}$ " phase was the intervention of the two mental skills that were identified as the participant's "strengths," and the " $C$ ” phase was the intervention of the two mental skills that were identified as the participant's “weaknesses.” The length of the baseline phases (“A” phases) was two weeks, or a minimum of four data points. The lengths of the "B" and " $C$ " phases were also two weeks or a minimum of four data points each. Since these 
phases included two mental skills interventions each (two strengths and two weaknesses), each mental skills intervention occurred for one week. The participant met with the researcher for one session per week for 20-40 minutes to learn how to use a mental skill and apply it towards injury rehabilitation and sport. Positive effects of a multimodal mental skills intervention with injured athletes (similar to the one in this study) have been found after one session of each intervention (Johnson, 2000).

The strengths of this design are that it is more manageable and practical to conduct a multimodal intervention with single subjects rather than a large group. This design provided the researcher with insight into the preferences and perceived effectiveness of mental skills interventions with injured athletes and helped to understand the importance of individual differences to take into consideration when crafting and conducting such interventions. However, a major limitation of this design is the lack of generalizability to the larger population of injured athletes.

The qualitative aspect of the study included the interviews (pre- and post-intervention), participant comments from the online journals or mental skills manual logs, and ATC comments from the surveys. The purpose of the pre-intervention interview was to help the researcher build rapport with participants and learn about participants’ background and injury experiences. The purpose of the post-intervention interview was to add to the interpretability of the quantitative data and allow the researcher to learn more about the participants’ preferences and experiences with learning the mental skills and applying them.

\section{Variables}

The independent variables were the four mental skills interventions of goal setting, relaxation, imagery, and self-talk. The eight dependent variables were: (1) self-reported use of 
each mental skill; (2) perceived effectiveness of each mental skill; (3) satisfaction with each mental skill intervention; (4) self-efficacy for rehabilitation; (5) self-efficacy for return to sport; (6) adherence to the rehabilitation protocol (athlete-rated and ATC-rated); (7) attitude during rehabilitation (athlete-rated and ATC-rated); and (8) speed of recovery.

All dependent variables, except satisfaction with the mental skills intervention, were measured at least twice per week during the study. Satisfaction with the mental skills intervention was only measured during the intervention phases of the study (weekly). A requirement of single-case designs is that data be measured continuously (at least several times a week) because such designs measure the effects of interventions on variables over time (Kazdin, 1982).

\section{Instrumentation}

Demographics and background. Demographics and background information for each participant was obtained through an initial semi-structured interview (see Appendix B) which was audio recorded. Participants provided information about their age, gender, sport played, athletic history, description of current injury, date of injury and surgery, thoughts about the current injury and rehabilitation so far, previous history with injuries, and previous history with sport psychology/mental training. Participants were asked to provide contact information on a separate form (i.e., e-mail address and phone number) so that they could be contacted by the researcher throughout the study to schedule weekly meetings and to send reminders to complete assessments. The contact information along with a confidential code number of the participant were stored separately from the rest of the data.

Mental skills. The athlete's previous use of mental skills in sport was assessed with the Test of Performance Strategies-2 (TOPS-2; Hardy, Thomas, Sheppard, \& Murphy, 2005; see 
Appendix C). The TOPS-2 is a 68-item instrument which measures an athlete's use of selected psychological skills and techniques in both practice and competition. Although participants completed the entire instrument, only total subscales scores for goal setting, self-talk, relaxation, and imagery were used because those were the four intervention skills in the study. Participants reported how frequently they used a technique on a 5-point ordinal scale consisting of 1 (never) to 5 (always).

The TOPS-2 is a modified version of the TOPS (Thomas, Murphy, \& Hardy, 1999), which was found to have problems with fit statistics from confirmatory factor analysis. As a result, in the second version of the test, the authors added and subtracted several items and added another subscale to competition strategies. The nine subscales that measure use of psychological techniques in competition are: self-talk, emotional control, automaticity, goal setting, imagery, activation, relaxation, negative thinking, and distractibility. There are four items per subscale, resulting in 36 items for the competition strategies. The fit statistics for the nine-factor model were excellent (e.g., CFI = 0.98; Hardy, Thomas, Sheppard, \& Murphy, 2005).

There are eight TOPS-2 subscales that measure use of psychological skills in the practice setting, which include: self-talk, emotional control, automaticity, goal setting, imagery, activation, relaxation, and attentional control. There are four items per subscale, resulting in 32 items for the practice strategies. The fit statistics for the eight-factor model were very good (e.g., CFI $=0.97)$. Cronbach's alphas were above 0.70 for all competition and practice subscales with the exception of the two competition subscales of automaticity (0.62) and distractibility (0.44). This was not a major concern for the present study because the current investigation focused on the scores for the practice and competition subscales of goal setting, relaxation, imagery, and 
self-talk and no inferential statistics were used to compare means or to correlate scores on these subscales.

Monitoring of mental skills intervention. The athletes’ use of each mental skill along with their perception of the effectiveness of the intervention and satisfaction with the intervention were measured as single items (see Appendix D). Participants were asked to consider use and effectiveness of the mental skills over the past few days (since they last filled out an assessment). Mental skill use and perceived effectiveness were measured at least twice per week. Satisfaction was measured once per week (only during the intervention phases). All items were measured on a 10-point ordinal scale. The items assessing use of the mental skills stated, "How much did you use (goal setting, relaxation, imagery, or self-talk) in relation to your injury rehabilitation?” Answers were on a scale from 1 (did not use at all) to 10 (used very much).

The perception of effectiveness items stated, "How much of an effect did (goal setting, relaxation, imagery, or self-talk) have on your injury rehabilitation?” over the past few days. Answers ranged from -5 (very negatively affected rehabilitation) to +5 (very positively affected my rehabilitation). Finally, the satisfaction item stated, "How satisfied were you with the mental skills intervention you learned this week?” Answers ranged from 1 (not satisfied at all) to 10 (extremely satisfied). Space was also provided for participants to comment on their use, perceived effectiveness, and satisfaction with the mental skill.

Self-efficacy for rehabilitation. The Athletic Injury Self-Efficacy Questionnaire (AISEQ; Milne, Hall, \& Forwell, 2005; see Appendix E) was used to measure the athlete’s self-efficacy in the rehabilitation setting. This is a 7-item instrument, which measures both perceived task and coping efficacy for rehabilitation. Task efficacy refers to confidence in one’s ability to successfully perform the required tasks in rehabilitation. An example of an item from this 
subscale is, "I am confident that I can perform all the required rehabilitation exercises.” Coping efficacy is confidence in one's ability to perform rehabilitation exercises in spite of barriers and obstacles. An example from this subscale is, "I am confident that I can do my rehabilitation exercises when I feel I do not have time.” Participants rated their self-efficacy for each item on a confidence scale ranging from $0 \%$ (no confidence) to 100\% (completely confident). Scores were averaged within each subscale to obtain the two self-efficacy scores (task and coping). Confirmatory factor analyses found there to be a two-factor solution, and the CFI indicated a good model fit (0.99). Cronbach alpha coefficients were reported to be 0.81 and 0.80 for the task and coping self-efficacy subscales respectively (Milne et al.). In this study, the researcher calculated and analyzed scores for each subscale separately.

Self-efficacy for return to sport. A modified version of the State Sport Confidence Inventory (M-SSCI; Magyar \& Duda, 2000; see Appendix F) was used to measure the selfefficacy of participants to perform successfully in future athletic events. This 13-item questionnaire was modified for injured athletes by asking them to "rate the confidence you feel since your injury in your ability to...” and respond to each item (e.g., execute the skills necessary to be successful, perform under pressure, and make critical decisions during competition) on a scale from 0 (not at all confident) to 100 (completely confident). The average of the 13 items was used as the final score. The M-SSCI was found to be internally reliable with alphas ranging from 0.97 to 0.98 at various assessment time periods (Magyar \& Duda). No measures of validity were reported in this study. However, satisfactory content, concurrent, and construct validity were reported for Vealey’s (1986) original version of the SSCI (Feltz \& Chase, 1998).

Adherence to rehabilitation. The Sport Injury Rehabilitation Adherence Survey (SIRAS; Brewer et al., 2000; see Appendix G) was completed by the participant's athletic trainer (ATC) 
to assess the athlete's adherence to the rehabilitation protocol. The SIRAS was also modified for the athlete to self-evaluate adherence (see Appendix H). The SIRAS consists of three questions on a 5-point ordinal scale, which assess participants’ intensity of completing rehabilitation exercises, frequency of following the ATC's instructions, and receptivity to change in the rehabilitation protocol. The total score of all 3 items was used as the adherence score. Brewer et al. (2000) provided evidence to support the unidimensionality, test-retest reliability (ICC $=0.77$ over a one-week period), and internal consistency (alpha $=0.82)$ of the SIRAS. They also found support of discriminant validity in that scores on the SIRAS were significantly correlated (with a low magnitude) with attendance at rehabilitation sessions $(r=0.21, p<.05)$. This statistically significant result indicated that there is some overlap between scores on the SIRAS and attendance at rehabilitation. However, the low positive magnitude of the correlation coefficient indicated that the SIRAS appears to provide unique information about adherence, over and above that of attendance at rehabilitation. Brewer et al. found there to be a moderate (0.57) intraclass correlation coefficient between the ratings of different service providers who assessed the same athletes. This correlation suggested that different service providers, such as athletic trainers, interpret and use the measure in similar ways; therefore, one can justify having several athletic trainers complete the SIRAS in one study.

Recovery time and attitude towards rehabilitation. A single item developed by the researcher (see Appendix G) was used to assess recovery time of the athlete compared to the norm on a 5-point ordinal scale ranging from 1 (significantly below average) to 5 (significantly above average). The ATC working with the athlete completed this item along with the SIRAS. Attitude towards rehabilitation was assessed by both the athlete and the ATC (see Appendices G and $\mathrm{H}$ ). Attitude was measured with a single item stating, "How would you rate your (or your 
patient's) attitude toward performing your (or his/her) rehabilitation exercises?” Answers ranged from 1 (very negative) to 7 (very positive) (Levy, Polman, \& Clough, 2008). The use of a single item to measure attitude has been supported in psychological research (Ajzen, 2002, as cited in Levy, Polman, \& Clough, 2008). All ATC-rated items were included in a survey entitled, Patient Evaluation Form (Appendix G).

Exit interview. After completion of the study, participants completed a semi-structured interview with a research assistant regarding their experiences with the mental skills intervention. Specifically, participants were asked likes/dislikes of the intervention, how they used each of the mental skills they learned during the study and how they may use the skills in the future, perceived effects of using the skills, and their thoughts about their rehabilitation experience in general. They were also asked to rank-order the skills in terms of which skills they liked (i.e., from most to least) and usefulness for the future. The interview was audio recorded (see Appendix I for exit interview script).

\section{Procedures}

Prior to the onset of the current study, a qualitative pilot study was conducted with two participants so that the researcher could become familiar with the intervention procedures and determine if any modifications were necessary. Two injured male athletes learned two mental skills each. Both responded favorably to the interventions, and slight modifications to the intervention and assessment materials were made in response to the pilot study. Please see Appendix J for details on the pilot study.

Institutional Review Board approval was obtained prior to conducting the study (see Appendix K). A letter of informed consent (see Appendix L) was signed by all participants prior to their participation in the study. The athletic trainers who worked with the participants in the 
study also signed a letter of informed consent (see Appendix M), as they had to complete measures assessing the participant. Individuals who agreed to participate in the study met with the researcher for approximately one hour to complete the TOPS-2, initial interview, and the first online journal. All online journals included: (1) the AISEQ; (2) M-SSCI; (3) monitoring of mental skills (use and perceived effectiveness); (4) athlete-modified SIRAS; and (5) attitude assessment. Also, all journals asked the participant to rate how difficult rehabilitation exercises have been on a scale of 1 (not difficult at all) to 5 (very difficult) and provided space for the participant to describe any significant events that have occurred over the past few days, such as developments in rehabilitation, issues with teammates or coaches, and anything going on at home, in school, with relationships, etc.

Participants were required to complete the online journal at least twice per week throughout the 8 weeks of the study. They were prompted through e-mail during the week to access the link where they could complete and submit their journals. Several measures were taken to prevent "response sets” and enhance compliance with filling out the assessments including: (1) the order of the questions was changed throughout the study; (2) a motivational quote selected by the investigator was included in each e-mail that was sent to the participant; and (3) the participants were asked to fill out a journal at the beginning of an intervention session if they had not completed all assessments for that week.

Baseline. During baseline, participants completed the online journal for two weeks or a minimum of four data points. Athletic trainers completed the Patient Evaluation Form at least twice per week. The researcher frequently visited the athletic training room to drop off surveys and collect data from the ATCs. 
Interventions. The TOPS-2 was scored by a research assistant. The researcher was blind to the results. From the combined practice and competition scores on the four subscales of goal setting, relaxation, imagery, and self-talk, the participant was assigned two "strengths" and two "weaknesses" in terms of the use of those four mental skills. Since scores could turn out to be identical, the researcher added a ranking measure to break a "tie” (see Appendix N). The assistant randomly assigned the strengths or weaknesses phase to go first, and informed the researcher which two skills to teach in the first intervention phase and which two skills to teach in the second intervention phase. In the "strengths" phase, the researcher taught the strongest skill first and the next strongest second. In the "weaknesses" phase, the researcher taught the weakest skill first and the next weakest second, with the exception of P1 who was taught the weakest skill second in error.

A binder was provided to each participant to store all intervention materials in. Materials were provided each week during the approximately 20-40 minute meetings which took place in a private office. Participants learned one mental skill each week for a total of four weeks in the two intervention phases. The sessions were interactive and the structure included the teaching of the skill followed by practice exercises. After each skill was learned, participants were told that mental skills are most effective when practiced daily and were encouraged to practice these strategies both during rehabilitation and at home. Logs were included at the end of each mental skill's unit for the participant to record the use of that skill for the week. All intervention materials were individualized to the person’s specific injury and sport. See Appendix O for brief outlines of what each intervention session entailed and Appendix P for sample intervention materials. 
One intervention was goal setting. The participant was taught the principles of goal setting and how to set daily, weekly, and long-term goals for both rehabilitation and sport. Written goal setting exercises were done in this session. Another intervention was self-talk. During this session, participants were taught what self-talk is and how it could affect mood and performance. They were taught how to change and reframe self-talk to be most useful to performance (both in rehabilitation and sport). Participants completed exercises where they reframed self-talk statements and created an affirmation statement. A third intervention was relaxation. The participant learned and practiced two relaxation techniques in the session diaphragmatic breathing and passive relaxation. They received a CD of the passive relaxation script with tranquil music. The final intervention was imagery. Participants were taught how to use imagery in rehabilitation and sport, including where, when, and how imagery should be done. Imagery exercises of soothing imagery, rehabilitation imagery, and sport performance imagery were done during this session.

Throughout the intervention phases, participants continued to complete the online journals at least twice per week. These consisted of the same assessments as baseline, with the addition of evaluating their satisfaction with the mental skill taught to them that week in one of the journals during the week. ATCs also completed the Patient Evaluation Form at least twice per week.

Second baseline and final assessment. After the intervention phases were complete, participants continued to complete the online journals that were identical to the journals during baseline at least twice per week for two more weeks, or for a minimum of four data points. ATCs also completed the Patient Evaluation Form at least twice per week. Finally, during the eighth week of the study, the participant met with a research assistant to complete the exit interview. 


\section{Data Analyses}

Graphs of the data for the dependent variables were created using Microsoft Excel to provide a visual depiction of the data over the approximately eight week period of time from the start of baseline to the end of the second baseline phase. To determine the effects of the intervention, graphs were visually inspected for changes in mean, level, trend, and variability between phases. Graphs were also examined for overlap of data points between phases to determine if meaningful change occurred.

Kazdin (1982) defines changes in mean across phases as shifts in the averages of data for that phase. Changes in level refer to a shift in the data from the end of one phase to the beginning of the next phase. Changes in trend refer to "the tendency for the data to show systematic increases or decreases over time” (p. 235). Finally, visual inspection of intervention effects can be done by examining changes in the variability, or stability, of the data between phases.

Content analysis (Patton, 2002) was performed on the qualitative data in the study. All interviews were transcribed verbatim. The researcher and two research assistants independently coded the data from the exit interviews. The reviewers then met to discuss any discrepancies and develop a list of themes in the data. The researcher analyzed the data both within and across cases for patterns that emerged. Data triangulation was accomplished by comparing the exit interview data with information from the online journals, ATC surveys, and written material in the mental skills manuals. Investigator triangulation was also used as a check during analysis.

\section{Results}

Analyses revealed that participants varied in their responses to the interventions. Some participants demonstrated meaningful changes in their self-reported use of the mental skills in rehabilitation over the course of the study. The pattern in which participants rated the 
effectiveness of the mental skills on rehabilitation often corresponded to the pattern in which participants reported that they used the skills (i.e., if participants reported high use of a mental skill, they also reported high effectiveness; if they reported low use, effectiveness was lower). Participants showed few meaningful changes over the course of the study with respect to the other dependent variables. In this section, results will be presented for prior mental skill use in sport, and then the quantitative data obtained from the online journals will be summarized for each participant by variable in the following order: (1) self-reported use of mental skills;

(2) perceived effectiveness of mental skills on rehabilitation; (3) satisfaction with mental skills interventions; (4) self-efficacy for rehabilitation; (5) self-efficacy for return to sport;

(6) adherence; (7) attitude; and (8) speed of recovery. Qualitative data from the exit interview, online journals, and manual logs will be used to supplement the quantitative results throughout the section.

\section{Mental Skill Use in Sport}

Participant data from the Test of Performance Strategies-2 (TOPS-2; Hardy, Thomas, Sheppard, \& Murphy, 2005) are presented in Table 2. The assignment of skills as strengths or weaknesses is noted in parentheses next to each score. The table also includes which skills (strengths or weaknesses) were taught first. All participants scored the lowest on relaxation and differed in rankings for the other skills.

\section{Self-reported Use of Mental Skills in Rehabilitation}

The self-reported use of mental skills in rehabilitation is displayed graphically in Figures 1a through 12a. The skills that were identified as strengths are graphed separately from those that were identified as weaknesses. "Use of mental skills” emerged as a theme in the qualitative data, and included when and how the participant used the mental skill over the course of the study. 
Several graphs showed meaningful changes between phases. As seen in Figures 1a and 2a, P1 learned weaknesses first (goal setting and relaxation) followed by strengths (self-talk and imagery). While the weaknesses were being taught ( $\mathrm{C}$ phase), use of both of his strengths displayed a decreasing trend and then recovered to the baseline level in the B phase. Selfreported use of both skills decreased in variability in the B phase (when taught) and remained stable into the second baseline. The reported use of self-talk (strongest skill) showed more change in relation to the intervention than imagery, as seen by the rise in level and decrease in variability. In the exit interview, P1 said he used self-talk in rehab "all the time.” An example of self-talk was telling himself, “just to keep goin... and eventually...it will be OK.” With regards to imagery, P1 said he frequently used performance imagery, even before the intervention, to see himself “coming back 100 percent and imagine celebrating a winning goal.”

Participant 1's reported use of relaxation (weakest skill) also appeared to have responded to the intervention. His reported use of relaxation increased immediately post-intervention and then stabilized in phase B and the second baseline. Data from the online journal, relaxation log in the manual, and comments in the exit interview supported P1's use of relaxation, in that he said he used the deep breathing in rehabilitation during painful massages of his scar and while icing. His use of goal setting showed no change in level throughout the study, but decreased in variability in phase C (when taught) and remained stable for the remainder of the study. P1's comments from the interview confirmed that his use of goal setting did not change in the longterm as a result of the intervention by saying that he does not sit down and formally set goals.

Participant 4 also learned his weaker skills before stronger skills (see Figures 7a and 8a). Self-reported use of his strongest skill, goal setting, was high at baseline and also displayed a small downward trend during the $\mathrm{C}$ phase, similar to $\mathrm{P} 1$. His reported use then stabilized in the $\mathrm{B}$ 
phase (when taught) at a lower level than baseline and remained stable to the second baseline. Reported use of his other strength, self-talk, was significantly lower than goal setting at baseline. Reported use of the skill increased after the skill was taught in the B phase and increased in level into the second baseline. P4's reported use of self-talk showed more change after the intervention than use of goal setting. In the exit interview, P4 mentioned several times that he used thought stopping, and that is what stood out the most to him from the interventions. He stated, “...that helped out a lot...there was one where it was like a three step thing. It was like say something, do something, and then think of something else, like think of a positive subject and that's what I’ve been doing lately.” He said that he used this process for rehab as well as family issues. He also said that he frequently used the affirmation statement he created during the intervention, "You're the man!"

Similar to P1, P4's reported use of relaxation (weakest skill) changed more following the intervention than his use of imagery. His reported use of relaxation showed an increasing trend after the skill was taught in the $\mathrm{C}$ phase, and then stabilized at a higher level in the B phase and second baseline. After imagery was taught, P4's reported use of the skill stabilized, however there was no change in level throughout the 8 weeks. In the interview, P4 said he used the deep breathing (relaxation) before going to sleep and when working on schoolwork. He also said that he used performance imagery to see himself back in shape and wrestling again.

Participant 6 learned the skills that were assigned as strengths before weaknesses (see Figures 11a and 12a). His reported use of goal setting was high at baseline and remained high throughout the study. Therefore, there appeared to be no meaningful change in use as a result of the intervention. In the interview, P6 said he frequently used goal setting for setting "little specific goals” for rehab. He gave an example of a performance goal that he set concerning his 
rehab exercises. His other strength, self-talk, appeared to respond to the intervention. His reported use of self-talk decreased in variability and stabilized at a higher level throughout the study after being taught. However, in the interview, P6 said he did not use self-talk during rehabilitation.

For the skills assigned as weaknesses for P6, imagery increased in level in the C phase (when taught) and remained high into the second baseline. However, he said in the interview that he used imagery only “a little bit,” trying to picture himself getting “better faster...in rehab.” Reported use of relaxation did not appear to change as a result of the intervention, as use of this skill was already high in the B phase. P6's responses in the interview confirmed this, as he said he used relaxation “a lot” and “always” had. He said he used deep breathing for stress management with rehabilitation and school.

P2 and P5 reported low use of all mental skills throughout the study. Both participants learned strengths first, which were imagery and goal setting (see Figures 3a and 9a). P2 reported some use of both skills in phase B after they were taught, then reported nonuse in all phases thereafter. This pattern was confirmed from comments in the online journal that was completed shortly after the intervention. P2 said he "visualized my ACL healing, and I also visualized what it would be like to play next fall." Shortly thereafter, he said he "hardly used it at all since last time I used it.” From the online journal and interview, P2 said he set performance goals for duration of riding the bike in rehab; however, he said that goal setting in rehab is “...kinda tough because I don't really know like where I'm supposed to be because I have no idea about...ACLs and stuff.” He did say that he started using goal setting with his academics. P5 echoed the thoughts of P2 regarding goal setting, saying, "I just kind of find it harder to do in physical therapy...to set my own goals, because to me, it kind of seems more up to the trainer to where 
I’m gonna get and like what I can do.” P5 also reported occasional use of imagery throughout the study after the skill was taught. In the week following the imagery intervention, P5 reported in the online journal that he "had a dream last night that I was in my first wrestling match since surgery and I won by pin. I think it might be due to the whole imagery thing since I’ve been doing it a little more.”

For the weaknesses (see Figures 4a and 10a), P2 reported occasional relaxation use in phase $\mathrm{C}$ after the skill was taught. There was no reported use of self-talk throughout the study. In the interview, P2 said he would take a deep breath before doing lifting exercises. He also said he practiced deep breathing and listened to the music on the relaxation CD while doing schoolwork and falling asleep. He said that he did not use the self-talk skill during rehab, but the intervention made him realize how much he used it in sport, specifically that his negative self-talk would be a detriment to his performance. P5 did not show meaningful change between phases in reported use of either relaxation or self-talk. However, relaxation was reported as used more than selftalk. Interview data and one comment from the online journal supported this, as he said the team did a relaxation exercise after lifting with a sport psychology consultant several times.

As seen in Figures 5a and 6a, P3 reported consistently high use of all four mental skills throughout the study and displayed ceiling effects for the skills of self-talk (strength) and goal setting (weakness). While imagery and relaxation levels were below that of self-talk and goal setting, reported use of all four were high and did not show meaningful changes between phases. The interview data supported this, as P3 stated, "I mostly use goal setting and self talk a lot, more than the other two.” She reported setting performance goals with target dates for rehab, specifically running. She cited examples of reframing her self-talk in rehabilitation to manage frustration as well as for school frustrations. For example, in the self-talk log from the manual, 
she wrote, "this will just help me get better" and said it was useful self-talk for her. P3 also mentioned in the interview that she used instructional self-talk prior to the intervention during cheerleading routines. In both the imagery log from the manual and interview, she reported using performance imagery of herself cheering either in practice or competition. Although P3 reported high use of relaxation in the quantitative data, during the interview, she gave examples of telling herself to relax (rather than the deep breathing or passive relaxation that was taught during the intervention).

Perceived Effectiveness of Mental Skills

The perceived effectiveness of each mental skill on the rehabilitation process is displayed graphically in Figures 1b through 12b. Skills that were identified as strengths are graphed separately from those identified as weaknesses. Graphs of perceived effectiveness are displayed with corresponding graphs of self-reported use of mental skills so patterns could be compared. Mostly, the patterns of perceived effectiveness followed similar patterns to the participants' selfreported use of the mental skills. Exceptions to this included the perceived effectiveness of selftalk (strength) and imagery (weakness) for P6 (see Figures 11a, 11b, 12a, and 12b).

"Effectiveness" also emerged as a qualitative theme, with subthemes of "efficacy” (i.e., whether or not the participant found the intervention to have an impact) and "outcome” (perceived effect that the skill had for the person). Most participants found at least some of the interventions to be helpful, while some reported that the interventions had little perceived impact (e.g., P5 and P6 for goal setting; P2, P5, and P6 for self-talk; and P2 and P6 for imagery, although P2 stated, "I think it would have a positive effect if I used it more”). Speaking about the interventions in general, P3 said, "I could take this with me and use it somewhere and like then...if someone else I know would get hurt or frustrated, I just want to be like, 'here, take this 
[binder with intervention materials], this could help you.”’ P5 concluded his interview with, “I think I actually benefited from doing this, just by, you know, talking to her and stuff like that...”

In the online journals and during the exit interview, participants reported both physical and psychological outcomes of using the mental skills. They reported that goal setting helped them progress through rehabilitation (P2, P3, P4, P5), motivated them in rehabilitation (P2, P3, P6), and helped build confidence (P4). For instance, P2 stated in the online journal, "I was able to push myself and get more out of my rehab.” Participants reported that using self-talk helped with motivation in rehabilitation and/or other areas of life (P1, P3, P4, P5), distraction from pain (P2), stress management/relaxation (P3), and focus (P5). For the skill of relaxation, participants said that the deep breathing helped with falling asleep (P2, P4), decreasing muscle tension and preventing soreness or fatigue after rehabilitation exercises (P4, P5), arousal regulation during lifting (P2), stress management (P6), or distraction from pain during rehab (P1). For instance, P1 stated, “...just take my mind off... what exactly was going on like during that rehab and...I guess it would just make it easier on me...with the pain...” P5 also commented on how doing the relaxation exercise after lifting with a sport psychology consultant “cleared our minds a little bit” and made him feel better and less tired after lifting. Finally, only P4 discussed outcomes of imagery for his rehabilitation, including helping to set goals ("It helps you with goal setting because like if you see what you want to do, then you can set a goal to that”) and building confidence to recover and return to sport.

\section{Satisfaction with Mental Skills Interventions}

Ratings of satisfaction with each mental skills intervention from the online journals are presented in Table 3. The overall group mean of satisfaction of weaknesses was higher than satisfaction of strengths. During the exit interview, participants were also asked to rank order the 
mental skills interventions in order of what they liked from most to least. These rankings did not always match the satisfaction ratings in the journals. For example, P1 gave goal setting the highest satisfaction score in the journals, but rated it as the skill he liked the least. Also, P2 and P3 ranked a skill significantly below the others (5 out of 10), and then ranked those skills as the one they liked second best during the exit interview. As a group, during the exit interview ranking, participants ranked relaxation and imagery in the top two skills they liked more than the other two skills. Most participants ranked one strength and one weakness in the top two skills they liked, while P2 ranked his two strengths and P4 ranked his two weaknesses as the top two skills they liked.

“Thoughts" about the intervention (opinions) emerged as a theme in the qualitative data and related to satisfaction with the interventions. While opinions of the goal setting and self-talk units were mixed (i.e., some liked them, others were more neutral), all of the feedback about relaxation was positive. P3 stated, “Actually it was really cool because she had a CD, and I got to like listen to it and she did this really cool technique thing and it was really neat, like I thought it was gonna be kind of weird...but it actually wasn’t, it was very cool.” Most of the feedback on the imagery unit was positive as well, with the exception of P2, who did not like to use imagery because he had trouble seeing positive images all the time. P1 summed up his experience with the interventions by saying, “...overall...it was good. Um, before I was like, 'oh I don’t want to...do this,' but...in the end....with like the relaxation stuff and like those things that I definitely, I guess I learned from it, [I] didn’t really...use it before....and I thought it was...very interesting and thought it worked out pretty well.” 


\section{Additional Qualitative Data Regarding Mental Skills Interventions}

Another theme that emerged from the qualitative data was "learning," which included anything that participants said they learned from the interventions. In general, several participants said they already knew about some of the skills (P3 and P6). Others commented on how they learned to use the skill in a more in-depth way (P3 and P5). P5 said that what stood out to him from the whole experience was “...learning how...[to] make better use of it all. You know, use of imagery, relaxation, stuff like that.” For goal setting, P4 learned that writing goals down is important. For self-talk, P2 and P4 said they learned how to keep self-talk positive, with P4 specifically talking about the three step process of thought stopping. P1 and P3 learned to avoid telling oneself "not” to do something, but rather tell oneself what to do. For imagery, P1 and P3 discussed learning the two different perspectives of imagery (i.e., internal and external).

The final theme that emerged from the qualitative data was "future use" of mental skills, which referred to how the participant may use the skill(s) in the future. For goal setting, participants noted that they would use it when they returned to sport (P2, P3, P4, P5, P6). Specifically, P4 and P5 gave an example of setting outcome goals for sport, and P5 also said he would set short- and long-term goals for sport when he returned. P3 said that as she returned to sport, she would set performance goals to gradually get her tumbling skills back. P2 noted that he would use goal setting for jobs in the future, and P6 said that he would set goals for schoolwork. For self-talk, participants said they would use it when they return to sport for arousal regulation (P3), to stay positive before competition (P5), and in a "hard situation" during a match (P6). P4 also said he would use reframing when he had a negative thought, specifically about his injury. For relaxation, participants said they would use it in sport before competition (P1, P2, P5), in school before tests (P1, P5), and to manage stress (P4, P6). Finally, for imagery, P1 and P3 said 
they would use performance imagery of being successful in sport. P3 said that she would specifically use internal imagery (imagining the cheerleading routine from her own viewpoint). P4 said he would use imagery for setting goals to help him get back into wrestling.

During the exit interview, participants were asked to rank the four skills in order of usefulness for the future. As a group, participants ranked goal setting and imagery in the top two skills they would use in the future more than the other two skills. Most participants ranked one strength and one weakness in the top two skills they would use the most in the future, with the exception of P1 who ranked his two strengths as the top two skills he would use.

\section{Self-efficacy for Rehabilitation}

For the most part, participants scored high in self-efficacy for rehabilitation throughout the study (i.e., 85 and above). Task efficacy (i.e., belief in ability to perform rehabilitation exercises) and coping efficacy (i.e., belief in ability to complete rehabilitation exercises despite obstacles) were graphed as separate variables and can be found in Figures 13 through 18. Several participants differed in task and coping efficacy levels. During the first baseline, P1, P2, P5, and P6 scored higher on task efficacy than coping efficacy. For P1 and P6, scores on coping efficacy met or exceeded the task efficacy scores during the first intervention and remaining phases. This occurred for P2 during the second intervention phase (phase C). For P5, task efficacy scores remained higher than coping efficacy throughout the study.

Meaningful changes across phases were seen for P1, P3, and P6. P1 showed a drop in level from the first baseline to phase $\mathrm{C}$ for both task and coping efficacy. During this phase, the athletic trainer working with the participant noted setbacks of a strained tendon and a personal issue. The participant also reported that he was sick. Task efficacy scores did not change for the rest of the study, while coping efficacy scores increased in mean and level during phase B and 
the second baseline. For both task and coping efficacy scores, P3 showed a decrease in variability and increase in mean and level from phase B to C and into the second baseline. P6 showed decreased variability in coping efficacy scores from the first baseline to all other phases of the study. Self-efficacy for Return to Sport

No meaningful changes across phases were found for any participant regarding selfefficacy for return to sport. For the most part, participants scored high on the M-SSCI (i.e., 85 or above), with the exception of P5, whose scores fell between 75 and 85 . All graphs for selfefficacy for return to sport can be found in Appendix Q. Adherence to the Rehabilitation Protocol (Athlete- and Athletic Trainer-rated)

P1 showed a drop in mean and level in self-rated SIRAS scores from the first baseline to phase C (see Figure 19). Scores did not show change throughout the rest of the study, except for an upward trend in the second baseline. For ATC-rated adherence, P3 showed an increase in level and decrease in variability in the middle of phase B, after self-talk was taught (see Figure 20). No other participants demonstrated meaningful changes in adherence scores throughout the study (see Appendix Q). For the most part, athletes and ATCs rated participants as high in adherence throughout the study (i.e., scores of 12 and above).

\section{Attitude During Rehabilitation (Athlete- and Athletic Trainer-rated)}

P2 displayed an increase in mean and level of self-rated attitude from phase A to B and maintained that level throughout the study (see Figure 21). During phase A, P2 reported in his journals that he struggled with exams in the beginning of the phase and was sick at the end. None of the other participants showed meaningful changes in self- or ATC-rated attitude between phases of the study (see Appendix Q). For P2 and P6 (self-rated) and P1 (ATC-rated), attitude 
scores followed very similar patterns to adherence scores throughout the study (see Figures 2123). Most athletes scored high for attitude (i.e., 5 and above with a ceiling of 7) when rated by self or ATC. Athletes and ATCs had comparable scores for adherence and attitude.

Speed of Recovery

None of the participants showed meaningful changes between phases for ATC-rated speed of recovery. All participants were rated average or above on their speed of recovery throughout the entire study (i.e., between 3 and 5). Graphs for speed of recovery can be found in Appendix Q).

\section{Discussion}

The purpose of this study was to examine individual athletes’ experiences with a multimodal mental skills intervention to provide insight into how to effectively craft an individualized mental skills program for sport injury rehabilitation from initial post-injury assessment. The central hypothesis was that the dependent variable scores (i.e., perceived effectiveness of mental skills, self-efficacy for rehabilitation, self-efficacy for return to sport, adherence, attitude, and speed of recovery) would improve as a result of the intervention, with greater changes during the phase that participants learned the mental skills that were identified as strengths. It was also hypothesized that DV scores would drop during the second baseline, but not as low as during the first baseline.

\section{Assessment of Mental Skill Strengths and Weaknesses}

In the present study, the TOPS-2 was administered to identify mental skills as strengths or weaknesses based on prior skill use in sport and competition. However, during baseline, some of the participants (P3, P4, P6) reported higher use of some of their "weaker” skills than their "stronger” skills. One explanation for this is that the difference in participants’ scores between 
strengths and weaknesses were small, so perhaps the TOPS-2 was not the most appropriate measure to use to determine strengths and weaknesses in mental skill use.

Another possible explanation is that the athletes did not find the skills that they applied in sport to be as applicable to injury rehabilitation. An example of this was found for goal setting. Both P2 and P5 identified goal setting as a strength, yet found it difficult to set rehabilitation goals because they felt that the goals were more up to the athletic trainer to set. Also, P2 said that learning about self-talk made him realize how much he used negative self-talk in sport and how it hurt his performance, but he did not find self-talk as important in injury rehabilitation. Future researchers should consider using multiple forms of assessment when identifying mental skill strengths and weaknesses. Perhaps researchers should look at self-reported use of mental skills during baseline to determine the mental skill strengths and weaknesses in regards to injury rehabilitation versus prior use in sport. An initial interview exploring the athlete's use of mental skills in sport and/or rehabilitation might also help clarify which skills the athlete uses more. It would also help to reveal intervention effects if exit interview data regarding the use of mental skills differed from initial interview data. McCann, Jowdy, and Van Raalte (2002) discuss the importance of using a battery of assessment techniques to develop a more comprehensive understanding of the athlete and form an appropriate plan of action for mental skills training. Self-reported Mental Skill Use Throughout the Study

It was specifically hypothesized that self-reported mental skill use of all skills would increase during the intervention phase in which the skill was taught. Also, use of the skills identified as strengths would respond better to the intervention than those identified as weaknesses. Although most participants reported some changes in skill use during the skill instruction phase, the nature of those changes varied. For example, several participants reported 
that they increased using a skill after it was taught (e.g., P1 and P4 for self-talk and relaxation, P2 for imagery and relaxation, P5 for imagery, P6 for self-talk and imagery). However, the increase in use was not consistently for strengths or weaknesses only. Some participants seemed to respond more to the intervention with one strength and one weakness (P1, P4, P5, P6).

Other participants showed decreased variability in self-reported use after the skill was taught (P1 for all skills; P4 for goal setting, relaxation, and imagery; P6 for self-talk). Perhaps participants began using a skill more consistently after it was taught to them. On the other hand, P4 reported a decrease in goal setting and imagery use after the skills were taught. One possible explanation for this is that after learning the details of the skills, he realized that he had not actually been using it as much as he thought he was. Another explanation might be that since he reported higher use of the other two skills (self-talk and relaxation) after they were taught, his use of the goal setting and imagery suffered.

P1 and P4 both learned the skills identified as weaknesses first and displayed a decrease in use of their stronger skills in the first intervention phase when the other skills were taught. Perhaps these participants were focusing more on using the skills that were taught in that phase and less on the other two skills. It is possible that the use of all four mental skills at the same time could be overload and present an unreasonable challenge to athletes. Having the athlete focus on practicing only two mental skills rather than four throughout their recovery could be a more effective approach.

Some participants reported extensive use of the skills throughout the course of the study (P3 for all skills, P6 for goal setting and relaxation). Perhaps due to ceiling effects, increases in the variable were unable to be detected. The qualitative data were useful for understanding this finding. For example, P3, who reported high frequency use of all skills, still described things she 
learned and would use as a result of the intervention, including how to make her self-talk and imagery more effective. However, P6 did not report as much learning as P3 and seemed to make more use of the skills that he already practiced prior to the intervention (goal setting and relaxation), as evidenced by his statements in the exit interview. Although P6 reported higher use of self-talk and imagery after the skills were taught, he said in the exit interview that he did not use those skills much.

In contrast, other participants reported limited practice of mental skills throughout the study. For example, P2 and P5 had goal setting as one of their stronger skills; however, their reported use of goal setting in rehabilitation was low throughout the study. Both participants said in the exit interview that they found it hard to set goals for rehabilitation because they perceived that the athletic trainer was in control of setting the goals for the rehabilitation program (i.e., external locus of control for goal setting in rehabilitation). Some literature suggests that giving injured athletes input in the goal setting process can increase motivation to achieve the goals (Gilbourne \& Taylor, 1998). Gilbourne and Taylor state, “Although the specific goal structure may often need to be set by the... athletic trainer, the athlete can become integrated into the process by being encouraged to question and discuss a treatment plan” (p. 135). Athletic trainers may want to discuss rehabilitation goals with the athlete, making it a more collaborative process so that athletes can perhaps feel more invested and in control of at least some aspects of their rehabilitation progress.

Since P2 and P5 reported low use of mental skills throughout the study and still had positive outcomes (i.e., adherence, attitude, and recovery time), the use of mental skills may not be necessary for injury recovery, contrary to recommendations from the literature (e.g., Crossman, 2001; Petitpas \& Danish, 1995; Taylor \& Taylor, 1997; Weinberg \& Gould, 2007). 
These two participants were very compliant with the study requirements (i.e., completing assessments at least twice per week, attending intervention sessions) even though they reportedly did not frequently practice the mental skills in rehabilitation. The fact that these participants continued to be engaged in the study when reporting low use of mental skills shows that they were probably receiving some benefits from the interventions. This may have been learning skills that they could apply to other areas of their lives (as P2 stated he did) or learning skills that would be useful once they returned to sport (as both stated). Both participants rated their satisfaction with the interventions as high, so they may have enjoyed themselves in the process. Perceived Effectiveness of the Interventions

Participants perceived mental skills to be more effective when they reported that they used the skills more. Thus, if practitioners (i.e., sport psychology consultants, counselors, athletic trainers, coaches) can get athletes to use mental skills more frequently, athletes may perceive them to be useful and continue to practice them. Since direction of causality cannot be established, it is possible that athletes were using the skills that they believed were effective more often. While effectiveness ratings of the mental skills varied from neutral/no effect to very effective, none of the participants rated the skills as negatively affecting their rehabilitation, and they did not report this qualitatively either.

Participants reported several positive physical and psychological outcomes of using the mental skills, including motivation, building confidence, distraction from pain, improved sleep, decreased muscle tension, stress management, and arousal regulation. Other studies have also found that injured participants reported positive outcomes of practicing mental skills (Driediger et al., 2006; Evans et al., 2000; Handegard et al., 2006; Rock \& Jones, 2002; Roykulcharoen \& Good, 2004). 


\section{Satisfaction with Mental Skills Interventions and Future Use of Skills}

It was hypothesized that participants would be more satisfied with learning skills that were identified as strengths versus weaknesses. The mean satisfaction ratings from the online journals, however, showed a slightly higher mean for the weaknesses than the strengths. Perhaps participants enjoyed learning about things that they had not been exposed to before. Unlike the other skills, all participants had positive feedback about the relaxation unit, the skill that they all identified as using the least in sport prior to getting injured. Again, perhaps participants enjoyed the novelty of learning something they were not familiar with. Also, participants may have found the relaxation session refreshing during their busy schedule. All participants reported stresses and frustrations throughout the study, whether it was with their injury, school, personal issues, team issues, etc. Perhaps a stress management, rather than a more performance enhancement focused intervention as was done in this study, would be the intervention of choice for long-term injured athletes. Overall though, participants had favorable impressions of the experience of learning mental skills for injury rehabilitation, which has been found in other studies of psychological interventions for injured participants (Evans et al., 2000; Naoi \& Ostrow, 2008; Rock \& Jones, 2002).

When ranking the four skills from what they liked the most to least during the exit interview, the majority of participants rated one strength and one weakness in their top two skills. The skills they liked learning about the best did not always correspond with the skills they ranked as most useful for the future. Therefore, enjoyment of learning a mental skill does not always indicate that athletes will practice and apply that skill in the future. From the exit interview ratings, as a group, participants said they liked the relaxation and imagery units best but found goal setting and imagery to be most useful for the future. Since imagery fell into both 
of those categories, perhaps this supports the recommendation of Taylor and Taylor (1997) to include imagery as part of all Psychological Rehabilitation Programs (PRePs). However, individual differences in imagery ability (and ability with any mental skill) must be taken into consideration when designing an intervention. For example, P2 had trouble keeping images positive. P2 would need more practice and work with this skill, or another skill might be more beneficial to him. Practitioners who teach mental skills may want to ask their patients what they would consider most useful to their rehabilitation at the onset of treatment.

It is encouraging that the majority of participants said that they would use the skills when they returned to sport as well as areas outside of sport. Some behavioral theories include intention as a factor that will influence behavior (e.g., Theory of Reasoned Action; Ajzen, 1985). Future researchers who teach mental skills to facilitate injury rehabilitation may want to interview participants once they return to sport to evaluate the knowledge they retained about mental skills that were learned during rehabilitation and see if and how the athletes employed the skills once they returned to sport.

Overall, both the quantitative and qualitative data provided evidence that participants had varied responses to the four mental skills interventions. These differences occurred in relation to: (1) how frequently they used the skill; (2) how they used the skill (i.e., in and out of rehabilitation); (3) what they learned from the intervention; (4) how much they enjoyed learning about the skill; (5) the perceived physical and psychological effects of using the skill on rehabilitation; and (6) how they might use the skill in the future. Other studies in which mental skills interventions were taught to injured athletes also found that there was individual variability in how participants responded to the interventions (Evans \& Hardy, 2002b; Evans et al., 2000; Naoi \& Ostrow, 2008; Rock \& Jones, 2002). 
Self-efficacy, Adherence, Attitude, and Speed of Recovery

For the most part, the intervention did not appear to impact the variables of self-efficacy for rehabilitation, self-efficacy for return to sport, adherence, attitude, or speed of recovery. This finding differs from previous research that found mental skills interventions to improve some of these factors (Evans \& Hardy, 2002a; Ross \& Berger, 1996, Theodorakis et al., 1997;

Theodorakis et al., 1996). Other studies found self-reported use of mental skills in rehabilitation to be related to these variables (Scherzer et al., 2001; Sordoni, Hall, \& Forwell, 2002). Similar to the present study, Sordoni et al. found that participants scored high in self-efficacy for rehabilitation, and ceiling effects may have prevented finding significant relationships between self-reported imagery use and self-efficacy for rehabilitation. The majority of participants in this study scored high on all of these variables throughout the study, making it difficult to detect changes, if there were any, between phases. From these data as well as data in the initial interview, it appeared that all participants were very motivated to recover and return to sport, as well as confident in their ability to do so. It is also possible that the time commitments required of the study led to a selection bias of highly motivated and adherent athletes as participants (i.e., it took conscientious participants to complete the assessments and interventions for the eight weeks of the study).

Several participants (P1, P2, P6) scored higher on task efficacy than coping efficacy (the two subscales of self-efficacy for return to sport) in the first baseline, and then coping efficacy met or exceeded task efficacy levels during and after an intervention phase in the study. It is possible that the interventions helped athletes learn to cope with obstacles such as lack of time, negative mood, and discomfort. 
Personal and situational variables that were measured qualitatively may have impacted the participants' quantitative responses. For example, P1 showed a decrease in self-efficacy for rehabilitation and a decrease in self-rated adherence during the first intervention phase. During this phase, P1 and the ATC working with him mentioned a strained tendon, sickness, and personal issues the athlete was dealing with. In another case, P3 showed an increase in selfefficacy for rehabilitation during the second intervention phase. This increase may have been due to the intervention, but is unlikely since she reported using all four mental skills at a high level during baseline and throughout the study. The second intervention phase was also when she reported a lot of progress in rehabilitation to do more functional skills, such as running. She stated in her initial interview that working out was very important to her and it was difficult to be prevented from doing this while injured. Her ability to run, therefore, may have increased her self-efficacy for rehabilitation. In addition, P2 showed an increase in self-reported attitude from baseline to the first intervention phase. This increase may have been a result of the intervention; however, his reported use of mental skills in rehabilitation was low. He also reported concurrent academic stressors and sickness during the baseline phase.

These findings are consistent with those of Naoi and Ostrow (2008) who found that personal and situational variables may have impacted results, specifically mood changes as a result of cognitive and relaxation interventions. Evans and Hardy (2002b) also found that personal and situational variables appeared to account for emotional responses, rehabilitation adherence, and use of goal setting for participants in a goal setting intervention study. These findings support an integrated model of response to sport injury (Wiese-Bjornstal et al., 1998), stating that personal and situational variables exert their influence on cognitive, emotional, and behavioral processes throughout rehabilitation. 
There were no changes across phases in self-efficacy for return to sport. Since the study took place early in the rehabilitation of all participants, it is possible that this measure was not as relevant as it would have been later in the rehabilitation process as participants were closer to returning to sport. For example, Evans et al. (2000) found that toward the latter phase and during reentry to competitive sport, goal setting was used to enhance self-efficacy. Future researchers should consider examining this variable during an intervention study that is closer to the time when participants will be returning to sport.

In some cases, attitude and adherence followed similar patterns (P3 and P6 self-rated, P1 ATC-rated). Therefore, those with more positive attitudes in rehabilitation may be more likely to adhere to the rehabilitation protocol and show more effort in rehab, or vice versa. If athletic trainers or other practitioners working with injured athletes would like adherence to be high, helping the athlete to have a positive attitude during rehabilitation could be useful. Several participants mentioned that self-talk helped them stay more positive in general; for that reason, managing self-talk to be positive could be helpful for adherence in the athletic training room.

\section{Limitations}

There were several limitations of the present study that may have influenced its findings. As mentioned above, selection bias may have been a factor in the sampling, in that conscientious, motivated athletes are the ones who completed the study. Also, since this was a single subject study with only six participants, generalizability to the larger population of injured collegiate athletes cannot be established.

All measures were self-report. Participants may have responded in a socially desirable manner and may not have been totally honest. Participant recall may have also been inconsistent or impacted by unaccounted for factors (i.e., personal and/or situational factors). Although 
several versions of the online journals were used throughout the study, the repeated measures used in the study may have led to response sets by participants (i.e., testing effect), which may have led to the low variability that was observed for several variables in the study.

In addition to being self-report, the measures in the study used subjective rating scales. Single case designs have traditionally used overt behaviors that can be measured by an observer. However, as long as variables are operationally defined and measured, internal events can be included in behavioral analyses (Kennedy, 2005). To get a less subjective idea of behavior change, future researchers should measure overt behaviors with natural dimensional qualities, such as counting the number of exercises participants complete during rehabilitation to measure adherence. To measure use of mental skills, participants could tally and describe each time they use a mental skill inside or outside of rehabilitation. The logs in the manuals were meant to do this in the present study; however, the log was only for the week following the intervention. Also, the majority of participants did not make use of the logs, as this was an optional activity. The questions in the journals that measured self-reported use and perceived effectiveness of the mental skills asked participants to rate these in relation to rehabilitation. The questions did not address the use or perceived effectiveness outside of the rehabilitation setting. Several participants mentioned using the mental skills for purposes other than rehabilitation, including for schoolwork, sleep, relationship issues, and stress management. Therefore, the questions may not have accurately reflected the use and perceived effectiveness of the mental skills. On the other hand, the questions themselves could have cued the participants to use the skills throughout the study.

The principal investigator was the person conducting the intervention in this study, so demand characteristics may have been an issue. Participants may have answered both 
quantitative and qualitative data in a manner which would please the researcher (i.e., Pygmalion effect), especially since rapport was built over the course of the study. In terms of delivery of the intervention, although the principal investigator followed an outline, interactions with each participant were quite different, which could have affected participant responses to the interventions. Interventions were also brief and only taught once to each participant. In order for mental skills programs to be effective, skills need to be practiced and incorporated into athletes' routines (e.g., Crossman, 2001; Taylor \& Taylor, 1997; Weinberg \& Gould, 2007). Therefore, a longer, more consistent intervention focusing on fewer than four mental skills may have produced greater effects.

The final limitation has to do with the technology used in the study for submitting the online journals. Several participants (P1, P3, P6) had difficulties getting the surveys to load or submit through the internet. They reported that they tried filling out the survey several times, but it would not work and they gave up. Therefore, some data were missing for some of the participants and this frustration could have affected how the participants answered the questions. To accommodate these challenges, participants were permitted to copy the survey and paste it into an email. For P6, paper surveys were given to him for the last three weeks of the study. Strengths

There were several strengths for the present study. The study utilized mixed methods (i.e., single subject quantitative analyses and qualitative analyses), which allowed the researcher to obtain a more complete understanding of the data. By piecing together the data, interviews, and interactions during the intervention sessions, the researcher was better able to understand why the data may have turned out the way it did. The qualitative data from the journals and athletic trainer questionnaires also provided information to help explain the data. By using this 
methodology, individual differences in response to the mental skills interventions could be observed.

Measuring the self-reported use of mental skills over the course of the study helped the researcher determine if the changes in variables were actually due to the intervention or due to extraneous factors. Also, variables were measured several times per week, allowing the researcher to observe trends, variability, and daily fluctuations in the data. Finally, the study was unique in that: (1) strengths and weaknesses of mental skills were measured by assessment prior to the intervention and used to create the order in which participants learned the skills in the study; and (2) intervention materials and exercises were individualized to the participant. Practical Implications and Future Directions

Sport psychology consultants and future researchers may find it prudent to teach less than four mental skills to injured athletes, as participants may feel overloaded with learning the four skills and unable to effectively implement all of them into a mental rehabilitation program. Taylor and Taylor (1997) recommend picking one or two techniques based on each psychological need of the athlete; however, the athlete may have several needs to work on. Based on the results of this study, the researcher would recommend implementing two mental skills into a mental rehabilitation program: one participant-perceived strength and one participant-perceived weakness. This way, athletes can effectively apply a skill they are familiar with to injury rehabilitation and build on the use of that skill. While one participant (P6) in the study viewed already knowing about the skills in a somewhat negative light, other participants (P1, P3, P5) enjoyed learning more specifics about the skills and how to make better use of them. Since participants also said they enjoyed and benefited from learning something new, such as relaxation, the researcher would recommend teaching one skill that athletes are less familiar 
with. As individuals tend to vary in responses to interventions (e.g., Evans \& Hardy, 2002b; Evans et al., 2000; Naoi \& Ostrow, 2008; Rock \& Jones, 2002), sport psychology consultants and others working with injured athletes should be flexible and work with athletes to produce a mental rehabilitation plan that the athletes would be most invested in.

Athletic trainers can incorporate simple skills in rehabilitation to help athletes enhance motivation, build confidence, reduce stress, and manage pain. Participants reported that several of the psychological skills produced these positive outcomes for their rehabilitation. An example would be diaphragmatic breathing, an easy skill to teach athletes when they are in painful situations in rehabilitation. Having athletes participate in the goal setting process can also empower athletes to feel more in control of their rehabilitation. Helping athletes reframe self-talk to be more productive or positive may enhance their attitude and possibly adherence to rehabilitation.

Future researchers may want to use alternative assessment measures to determine skills that are strengths and weaknesses for participants, such as use of the skills at baseline and interviews. Assessment of psychological need (e.g., the Emotional Responses of Athletes to Injury Questionnaire; see Smith, 1999) would be an alternative way to determine which mental skill(s) to choose to teach injured athletes, similar to what Taylor and Taylor (1997) recommend. In addition, each mental skill has many different components to it. For example, imagery could include healing, soothing, or performance aspects. Relaxation could mean simple deep breathing, or a more complex exercise like autogenic training. Future researchers may want to assess athletes' abilities with these skills more thoroughly prior to planning mental skills interventions based on strengths and weaknesses. 
Selecting a different sample, such as a community population, may lead to more variability in the data in terms of outcome variables (i.e., self-efficacy, adherence, recovery time). This population might not be as motivated to return to physical activity as the collegiate athletes in the current study. Also, measuring home exercise adherence might show more variability than adherence in the athletic training room or physical therapy settings, as participants would need to be more self-motivated for home exercise activities. With the benefits of mental skills interventions reported by participants in this study as well as previous studies, expanding these types of intervention studies to the community population would be worthy of investigation. 


\section{References}

Ajzen, I. (1985). From intentions to action: A theory of focus on these important subgroups. In J. Kuhl \& J. Beckman (Eds.), Action-control: From cognition to behavior (pp. 11-39). Heidelberg: Springer.

Bandura, A. (1977). Self-efficacy: Toward a unifying theory of behavioral change. Psychological Review, 84, 191-215.

Brewer, B. W., Andersen, M. B., \& Van Raalte, J. L. (2002). Psychological aspects of sport injury rehabilitation: Toward a biopsychosocial approach. In D. L. Mostofsky and L. D. Zaichkowsky (Eds.), Medical and psychological aspects of sport and exercise (pp. 4154). Morgantown, WV: Fitness Information Technology.

Brewer, B. W., Jeffers, K. E., Petitpas, A. J., \& Van Raalte, J. L. (1994). Perceptions of psychological interventions in the context of sport injury rehabilitation. The Sport Psychologist, 8, 176-188.

Brewer, B. W., Van Raalte, J. L., Petitpas, A. J., Sklar, J. H., Pohlman, M. H., Krushell, R. J., \& Ditmar, T. D. (2000). Preliminary psychometric evaluation of a measure of adherence to clinic-based sport injury rehabilitation. Physical Therapy in Sport, 1, 68-74.

Crossman, J. (1997). Psychological rehabilitation from sports injuries. Sports Medicine, 23, 333339.

Crossman, J. (2001). Coping with sport injuries: Psychological strategies for rehabilitation. New York: Oxford University Press Inc.

Cupal, D. D. (1998). Psychological interventions in sport injury prevention and rehabilitation. Journal of Applied Sport Psychology, 10, 103-123. 
Cupal, D. D., \& Brewer, B. W. (2001). Effects of relaxation and guided imagery on knee strength, reinjury anxiety, and pain following anterior cruciate ligament reconstruction. Rehabilitation Psychology, 46, 28-43.

Driediger, M., Hall, C., \& Callow, N. (2006). Imagery use by injured athletes: A qualitative analysis. Journal of Sport Sciences, 24, 261-271.

Evans, L., \& Hardy, L. (2002a). Injury rehabilitation: A goal setting intervention study. Research Quarterly for Exercise and Sport, 73, 310-319.

Evans, L., \& Hardy, L. (2002b). Injury rehabilitation: A qualitative follow-up study. Research Quarterly for Exercise and Sport, 73, 320-329.

Evans, L., Hardy, L., \& Fleming, S. (2000). Intervention strategies with injured athletes: An action research study. The Sport Psychologist, 14, 188-206.

Feltz, D. L., \& Chase, M. A. (1998). The measurement of self-efficacy and confidence in sport. In J. L. Duda (Ed.), Advances in sport and exercise psychology measurement (pp. 65-80). Morgantown, WV: Fitness Information Technology, Inc.

Francis, S. R., Andersen, M. B., \& Maley, P. (2000). Physiotherapists’ and male professional athletes’ views on psychological skills for rehabilitation. Journal of Science and Medicine in Sport, 3, 17-29.

Gilbourne, D., \& Taylor, A. H. (1998). From theory to practice: The integration of goal perspective theory and life development approaches within an injury-specific goal-setting program. Journal of Applied Sport Psychology, 10, 124-139.

Handegard, L. A., Joyner, B. A., Burke, K. L., \& Reimann, B. (2006). Relaxation and guided imagery in the sport rehabilitation context. Journal of Excellence, 11, 146-164. 
Hardy, L., Thomas, P. R., Sheppard, K., \& Murphy, S. M. (2005). Test of performance strategies (TOPS): Further development using confirmatory factor analysis. Manuscript submitted for publication.

Ievleva, L., \& Orlick, T. (1991). Mental links to enhanced healing: An exploratory study. The Sport Psychologist, 5, 25-40.

Johnson, U. (2000). Short-term psychological intervention: A study of long-term-injured competitive athletes. Journal of Sport Rehabilitation, 9, 207-218.

Kazdin, A. E. (1982). Single-case research designs: Methods for clinical and applied settings. New York: Oxford University Press.

Kennedy, C. H. (2005). Single-case designs for educational research. Boston, MA: Allyn and Bacon.

Levy, A. R., Polman, R. C. J., \& Clough, P. J. (2008). Adherence to sport injury rehabilitation programs: An integrated psycho-social approach. Scandinavian Journal of Medicine and Science in Sports, 18, 798-809.

Magyar, T. M., \& Duda, J. L. (2000). Confidence restoration following athletic injury. The Sport Psychologist, 14, 372-390.

McCann, S. C., Jowdy, D. P., \& Van Raalte, J. V. (2002). Assessment in sport and exercise psychology. In J. L. Van Raalte \& B. W. Brewer (Eds.), Exploring sport and exercise psychology (pp. 291-305). Washington, DC: American Psychological Association.

Milne, M., Hall, C., \& Forwell, L. (2005). Self-efficacy, imagery use, and adherence to rehabilitation by injured athletes. Journal of Sport Rehabilitation, 14, 150-167. 
Myers, C. A., Peyton, D. D., \& Jensen, B. J. (2004). Treatment acceptability in NCAA Division I football athletes: Rehabilitation intervention strategies. Journal of Sport Behavior, 27, 165-169.

Naoi, A., \& Ostrow, A. (2008). The effects of cognitive and relaxation interventions on injured athletes’ mood and pain during rehabilitation. Athletic Insight, 10 (1). Retrieved May 4, 2008, from http://www.athleticinsight.com/Vol10Iss1/InterventionsInjury.htm.

Ninedek, A., \& Kolt, G. S. (2000). Sport physiotherapists’ perceptions of psychological strategies in sport injury rehabilitation. Journal of Sport Rehabilitation, 9, 191-206.

O’Conner, E., Heil, J., Harmer, P., \& Zimmerman, I. (2005). Injury. In J. Taylor and G.S. Wilson (Eds.), Applying sport psychology (pp. 187-206). Champaign, IL: Human Kinetics.

Patton, M. Q. (2002). Qualitative research and evaluation methods (3 ${ }^{\text {rd }}$ ed.). Thousand Oaks, CA: Sage Publications.

Petitpas, A., \& Danish, S. J. (1995). Caring for injured athletes. In S. M. Murphy (Ed.), Sport psychology interventions (pp. 255-281). Champaign, IL: Human Kinetics.

Rock, J. A., \& Jones, M. V. (2002). A preliminary investigation into the use of counseling skills in support of rehabilitation from sport injury. Journal of Sport Rehabilitation, 11, 284304.

Ross, M. J., \& Berger, R. S. (1996). Effects of stress inoculation training on athletes’ postsurgical pain and rehabilitation after orthopedic injury. Journal of Consulting and Clinical Psychology, 64, 406-410.

Roykulcharoen, V., \& Good, M. (2004). Systematic relaxation to relieve postoperative pain. Journal of Advanced Nursing, 48, 140-148. 
Scherzer, C. B., Brewer, B. W., Cornelius, A. E., Van Raalte, J. L., Petitpas, A. J., Sklar, J. H., Pohlman, M. H., Krushell, R. J., \& Ditmar, T. D. (2001). Psychological skills and adherence to rehabilitation after reconstruction of the anterior cruciate ligament. Journal of Sport Rehabilitation, 10, 165-172.

Smith, A. M. (1999). Assessing athletes through individual interview. In R. Ray \& D. M. WieseBjornstal (Eds.), Counseling in sports medicine (pp. 75-91). Champaign, IL: Human Kinetics.

Sordoni, C., Hall, C., \& Forwell, L. (2002). The use of imagery in athletic injury rehabilitation and its relationship to self-efficacy. Physiotherapy Canada, 54, 177-185.

Taylor, J., \& Taylor, S. (1997). Psychological approaches to sports injury rehabilitation. Gaithersburg, MD: Aspen Publishers, Inc.

Theodorakis, Y., Beneca, A., Malliou, P., \& Goudas, M. (1997). Examining psychological factors during injury rehabilitation. Journal of Sport Rehabilitation, 6, 355-363.

Theodorakis, Y., Malliou, P., Papaioannou, A., Beneca, A., \& Filactakidou, A. (1996). The effect of personal goals, self-efficacy, and self-satisfaction on injury rehabilitation. Journal of Sport Rehabilitation, 5, 214-223.

Thomas, P. R., Murphy, S. M., \& Hardy, L. (1999). Test of performance strategies: Development and preliminary validation of a comprehensive measure of athletes' psychological skills. Journal of Sports Sciences, 17, 697-711.

Vealey, R. S. (1986). Conceptualization of sport-confidence and competitive orientation: Preliminary investigation and instrument development. Journal of Sport Psychology, 10, 471-478. 
Weinberg, R. S., \& Gould, D. (2007). Foundations of sport and exercise psychology (4 $\left.{ }^{\text {th }} \mathrm{ed}\right)$. Champaign, IL: Human Kinetics.

Wiese, D. M., Weiss, M. R., \& Yukelson, D. P. (1991). Sport psychology in the training room: A survey of athletic trainers. The Sport Psychologist, 5, 15-24.

Wiese-Bjornstal, D. M., Smith, A. M., Shaffer, S. M., \& Morrey, M. A. (1998). An integrated model of response to sport injury: Psychological and sociological dynamics. Journal of Applied Sport Psychology, 10, 46-69. 
Table 1

Demographic Information of Participants

\begin{tabular}{|c|c|c|c|c|}
\hline Participant & Demographics & Sport & Injury & Sport Psych Training \\
\hline \multirow[t]{4}{*}{1} & Male & Soccer & Torn ACL; knee surgery (ACL & 1 session with psychologist \\
\hline & 21 years old & & reconstruction); expected recovery & (referred by athletic trainer); \\
\hline & Caucasian & & time of $6-9$ mos. & informal interactions with \\
\hline & & & & team sport psych consultant \\
\hline \multirow[t]{4}{*}{2} & Male & Soccer & Torn ACL and lateral meniscus; knee & None \\
\hline & 18 years old & & surgery (ACL reconstruction and & \\
\hline & Caucasian & & meniscectomy); expected recovery & \\
\hline & & & time of 6-9 mos. & \\
\hline \multirow[t]{3}{*}{3} & Female & Cheerleading & Weber $\mathrm{C}$ fracture and dislocation of & None \\
\hline & 19 years old & & left lateral ankle; ankle surgery & \\
\hline & Caucasian & & (internal fixation of fracture); & \\
\hline
\end{tabular}


4

Male

19 years old

Caucasian

5

$$
\text { Male }
$$

18 years old

Mexican-American

6

$$
\text { Male }
$$

21 years old

Caucasian
Wrestling

Torn labrum; shoulder surgery (labral None

repair w/ capsular shift); expected

recovery time of 5-8 mos.

Wrestling

Torn ACL; knee surgery (ACL

reconstruction); expected recovery

time of 6-8 mos.

Wrestling

Torn labrum (SLAP lesion); shoulder

surgery (labral repair); expected

recovery time of 6-8 mos.
None

Team presentations (mental training) from sport psych

consultant

Note. The above information was obtained from the participant in the initial interview. Athletic trainers provided additional information regarding the injury and surgery if necessary. 
Table 2

Test of Performance Strategies-2 (TOPS-2) Data

\begin{tabular}{llllll}
\hline P & Goal Setting & Self-talk & Relaxation & Imagery & Taught first \\
\hline 1 & $27(\mathrm{~W})$ & $30(\mathrm{~S})$ & $17(\mathrm{~W})$ & $29(\mathrm{~S})$ & Weaknesses \\
2 & $24(\mathrm{~S})$ & $22(\mathrm{~W})$ & $19(\mathrm{~W})$ & $25(\mathrm{~S})$ & Strengths \\
3 & $31(\mathrm{~W})$ & $33(\mathrm{~S})$ & $27(\mathrm{~W})$ & $35(\mathrm{~S})$ & Strengths \\
4 & $28(\mathrm{~S})$ & $27(\mathrm{~S})$ & $25(\mathrm{~W})$ & $26(\mathrm{~W})$ & Weaknesses \\
5 & $28(\mathrm{~S})$ & $26(\mathrm{~W})$ & $16(\mathrm{~W})$ & $29(\mathrm{~S})$ & Strengths \\
6 & $30(\mathrm{~S})$ & $27(\mathrm{~S})$ & $17(\mathrm{~W})$ & $17(\mathrm{~W})$ & Strengths \\
\hline
\end{tabular}

Note. $\mathrm{S}=$ TOPS-2 subscale scores that resulted in the skill being ranked as a strength; $\mathrm{W}=$ TOPS-2 subscale scores that resulted in the skill being ranked as a weakness. 
Table 3

Satisfaction Ratings of Each Mental Skills Intervention

\begin{tabular}{cllllllc}
\hline & \multicolumn{3}{c}{ Strengths } & & & \multicolumn{3}{c}{ Weaknesses } \\
\cline { 2 - 4 } \cline { 6 - 7 } P\# & 1 & 2 & Mean & & 1 & 2 & Mean \\
\hline 1 & ST: 8 & I: 8 & 8.0 & & R: 8 & GS: 9 & 8.5 \\
2 & I: 5 & GS: 9 & 7.0 & & R: 9 & ST: 10 & 9.5 \\
3 & I: 10 & ST: 5 & 7.5 & & R: 9 & GS: 9 & 9.0 \\
4 & GS: 10 & ST: 10 & 10.0 & & R: 10 & I: 10 & 10.0 \\
5 & I: 10 & GS: 10 & 10.0 & & R: 10 & ST: 10 & 10.0 \\
6 & GS: 9 & ST: 10 & 9.5 & & R: 8 & I: 10 & 9.0 \\
Overall Means & & 8.67 & & & 9.33 \\
\hline
\end{tabular}

Note. GS = goal setting; $\mathrm{ST}$ = self-talk; $\mathrm{R}$ = relaxation; $\mathrm{I}$ = imagery. Satisfaction ratings were obtained in the online journals during the week following when the intervention was taught, and were measured on a scale of 1 (not satisfied at all) to 10 (extremely satisfied). 
Figure 1a. Self-reported use of mental skills (strengths) for P1.

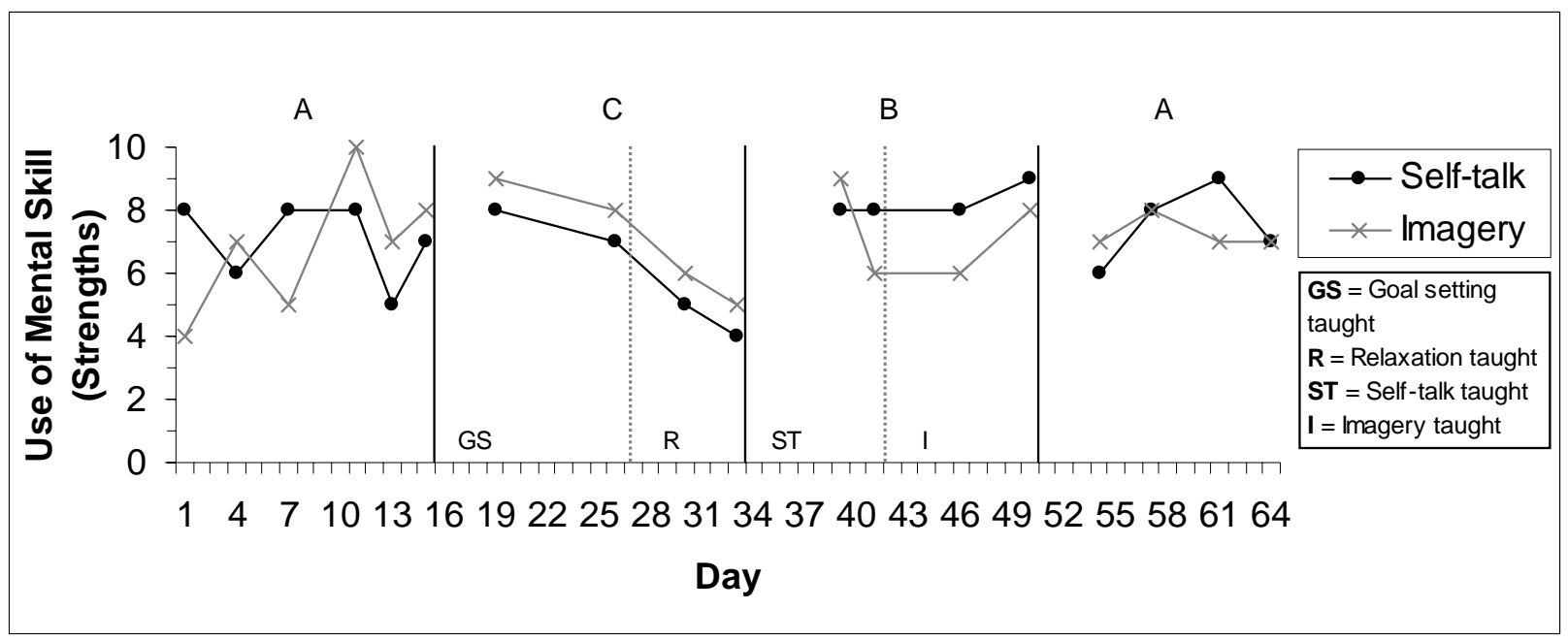

Figure $1 b$. Perceived effectiveness of mental skills (strengths) on rehabilitation for P1.

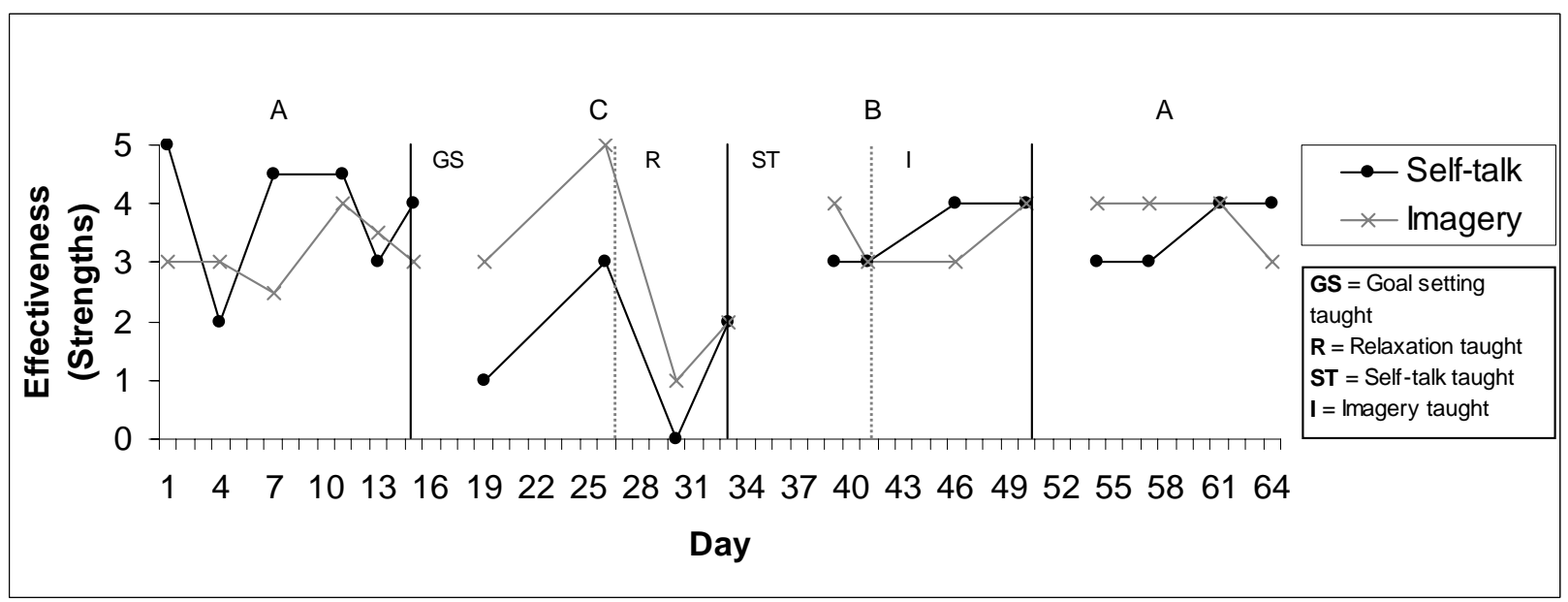


Figure 2a. Self-reported use of mental skills (weaknesses) for P1.

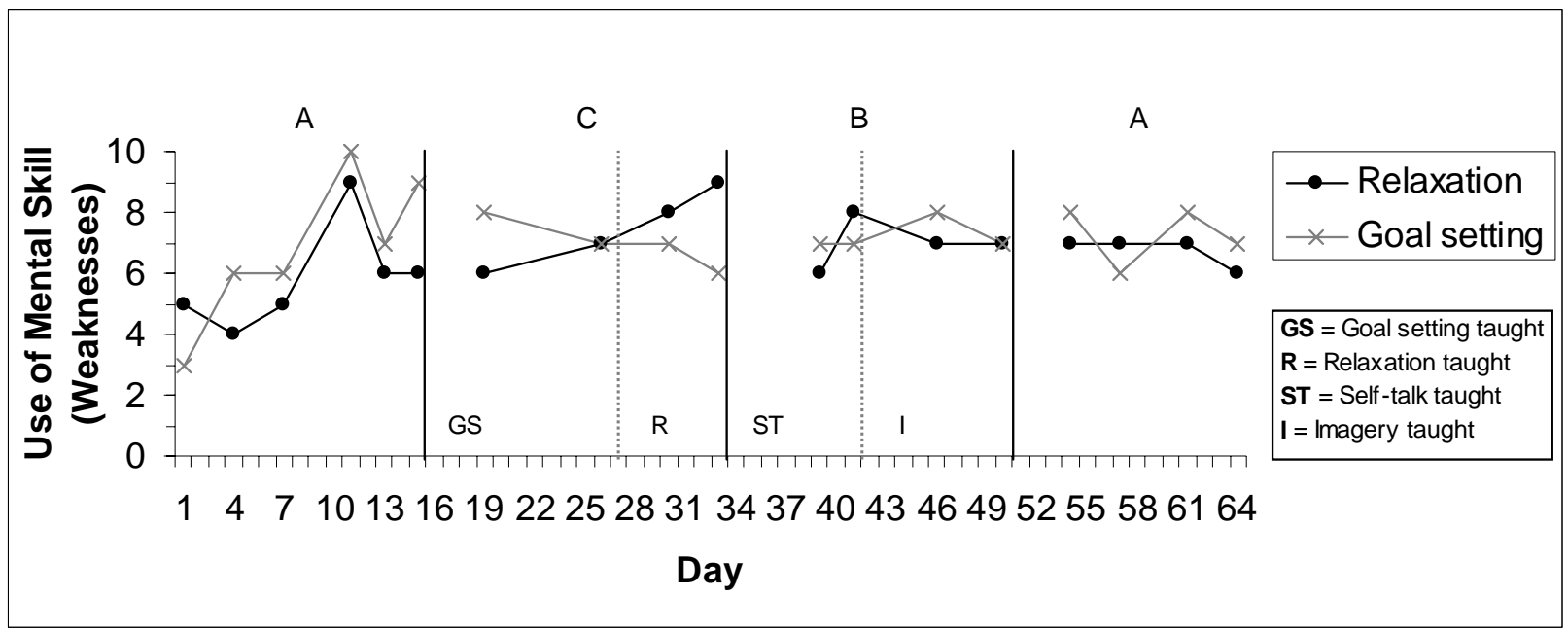

Figure 2b. Perceived effectiveness of mental skills (weaknesses) on rehabilitation for P1.

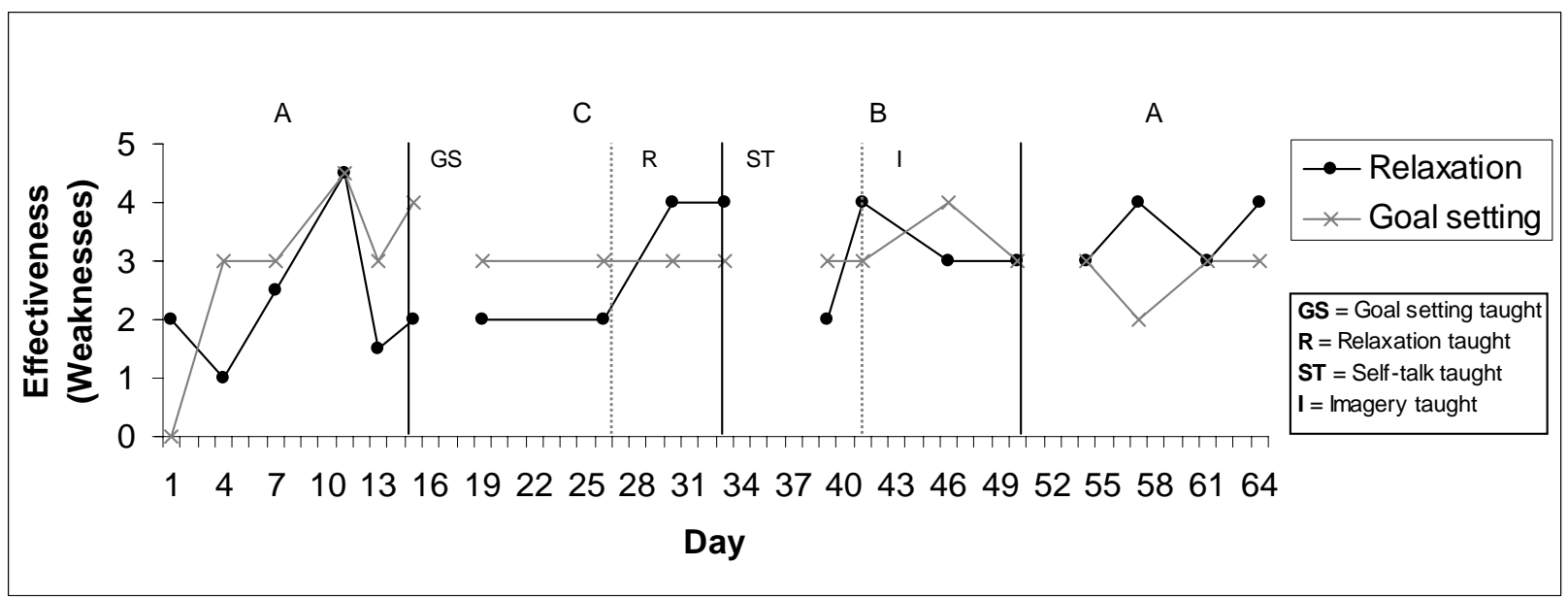


Figure 3a. Self-reported use of mental skills (strengths) for P2.

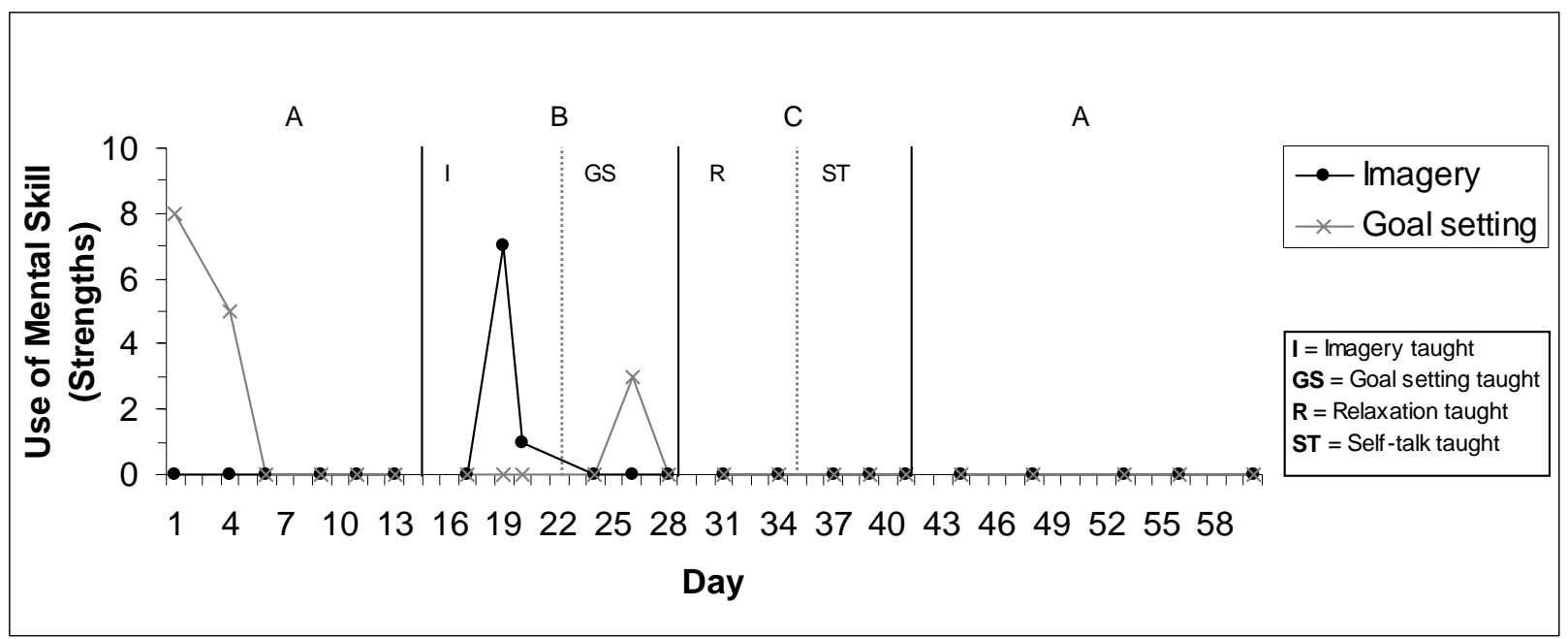

Figure 3b. Perceived effectiveness of mental skills (strengths) on rehabilitation for P2.

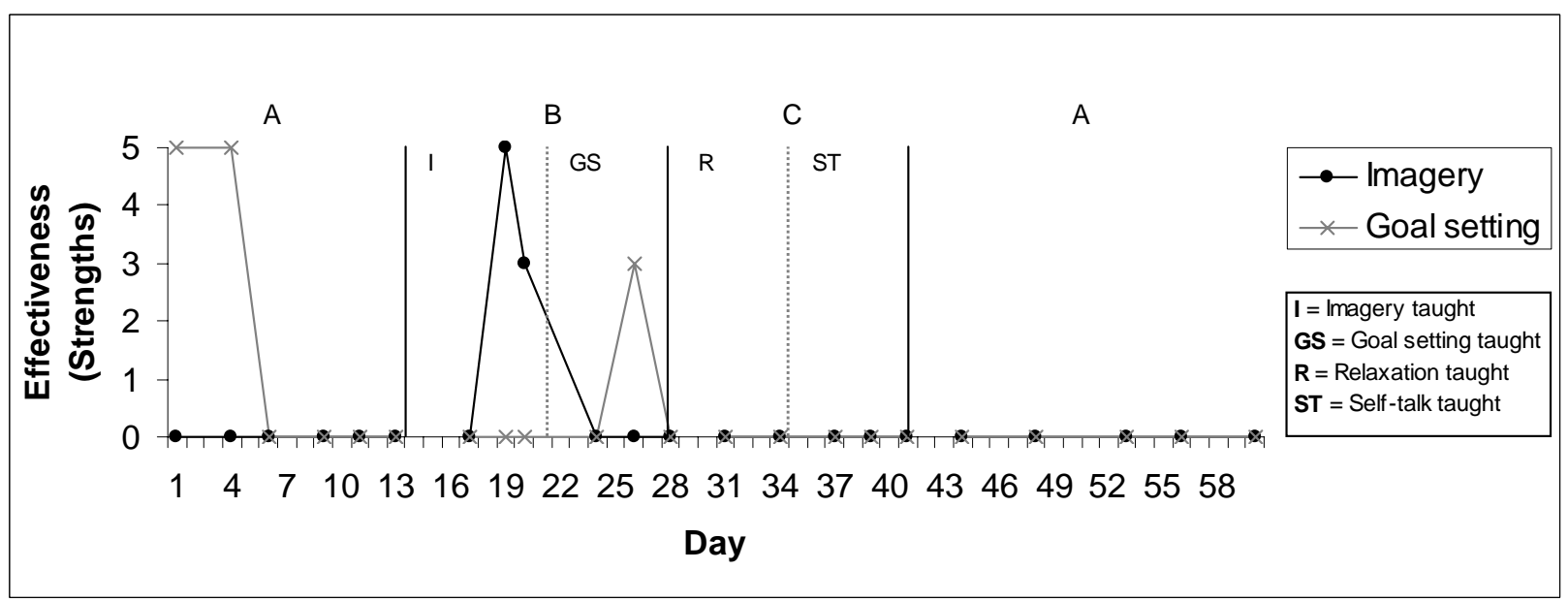


Figure 4a. Self-reported use of mental skills (weaknesses) for P2.

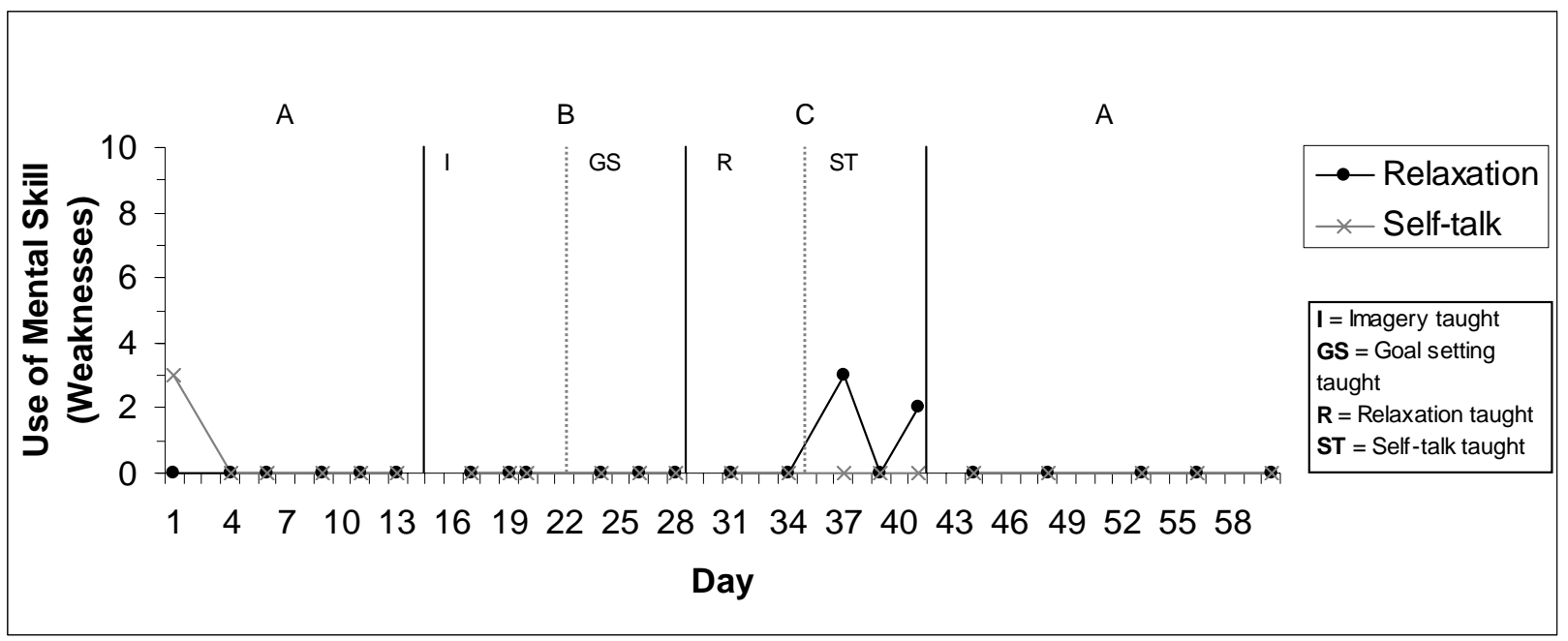

Figure $4 b$. Perceived effectiveness of mental skills (weaknesses) on rehabilitation for P2.

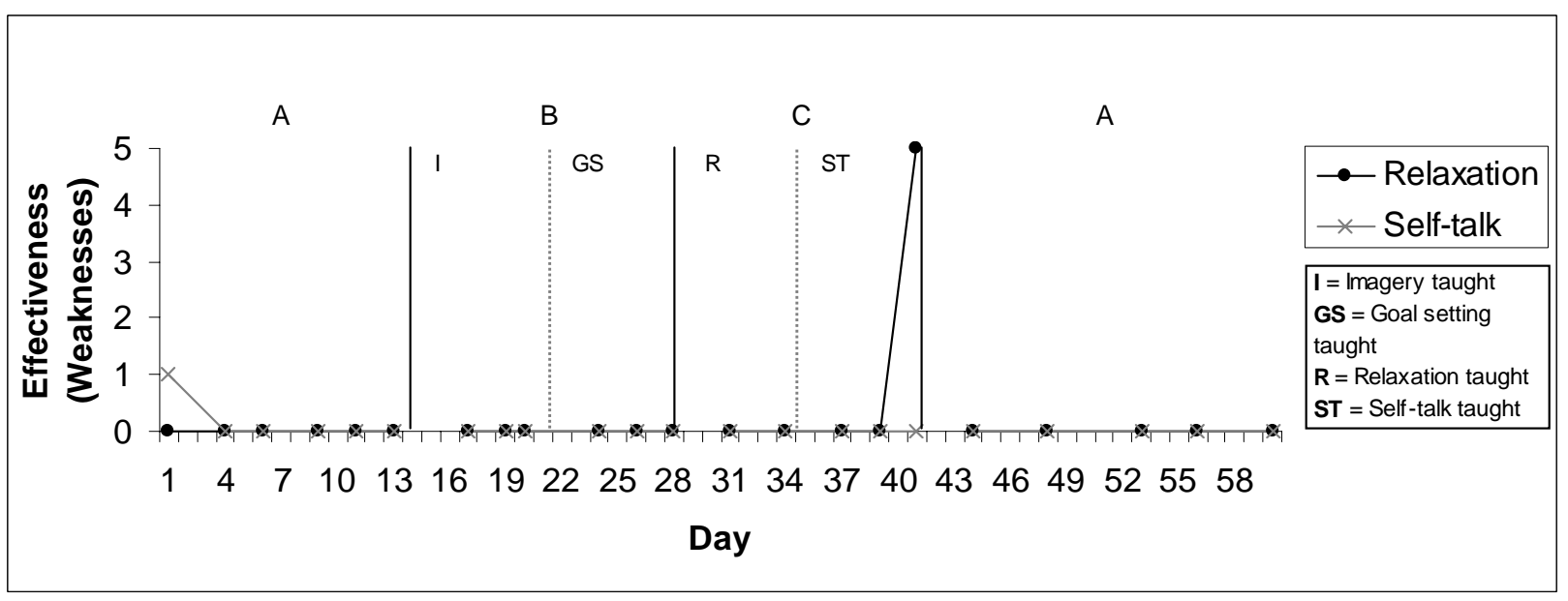


Figure 5a. Self-reported use of mental skills (strengths) for P3.

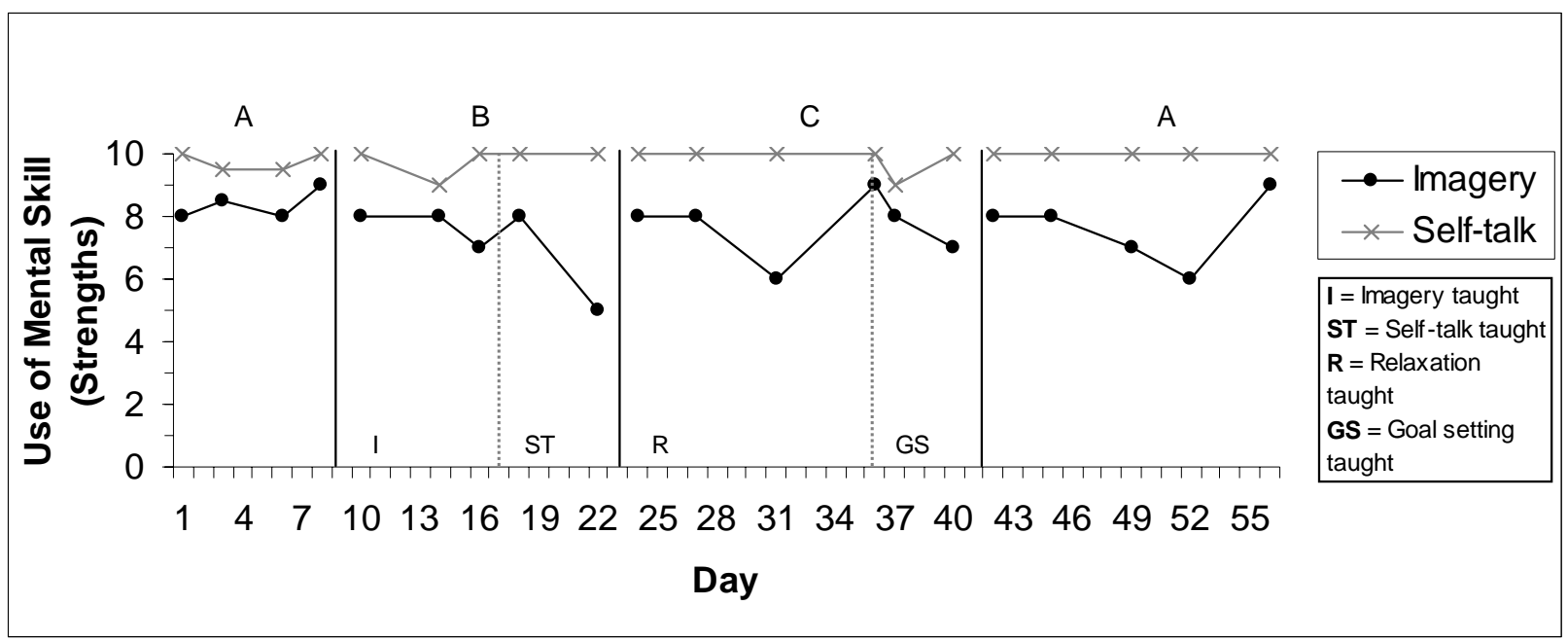

Figure 5b. Perceived effectiveness of mental skills (strengths) on rehabilitation for P3.

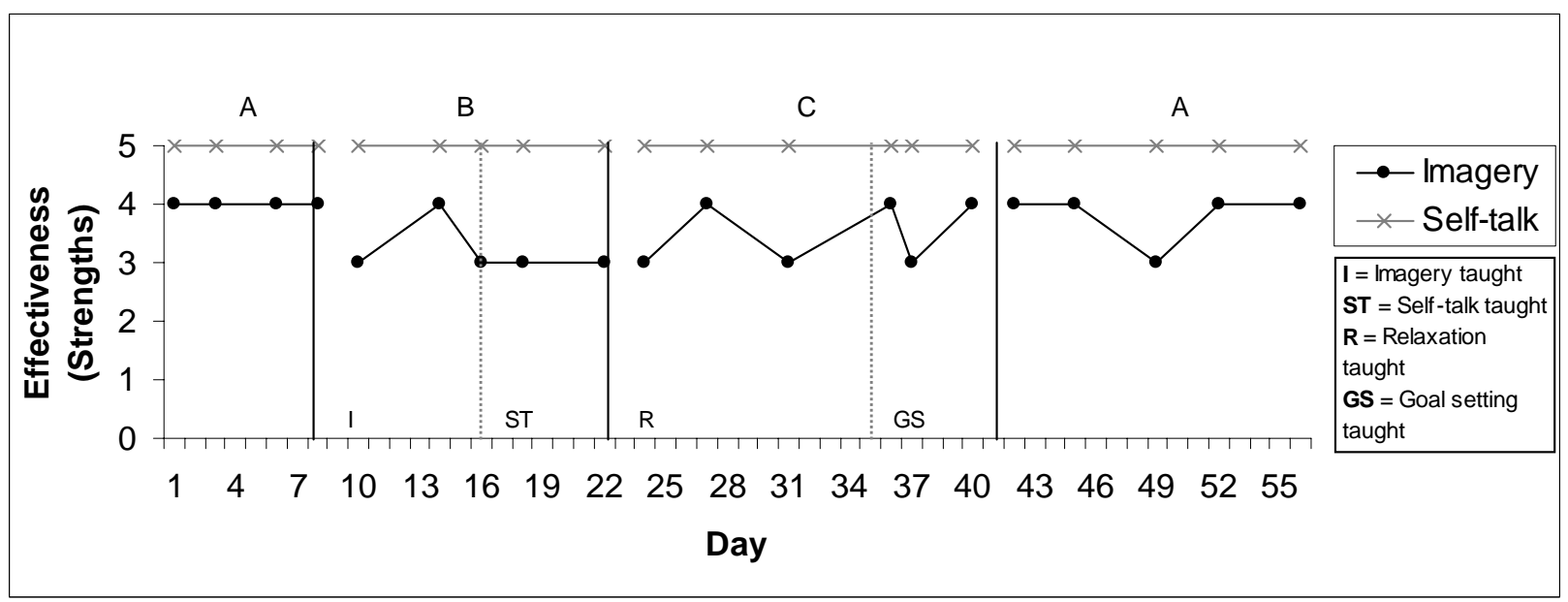


Figure 6a. Self-reported use of mental skills (weaknesses) for P3.

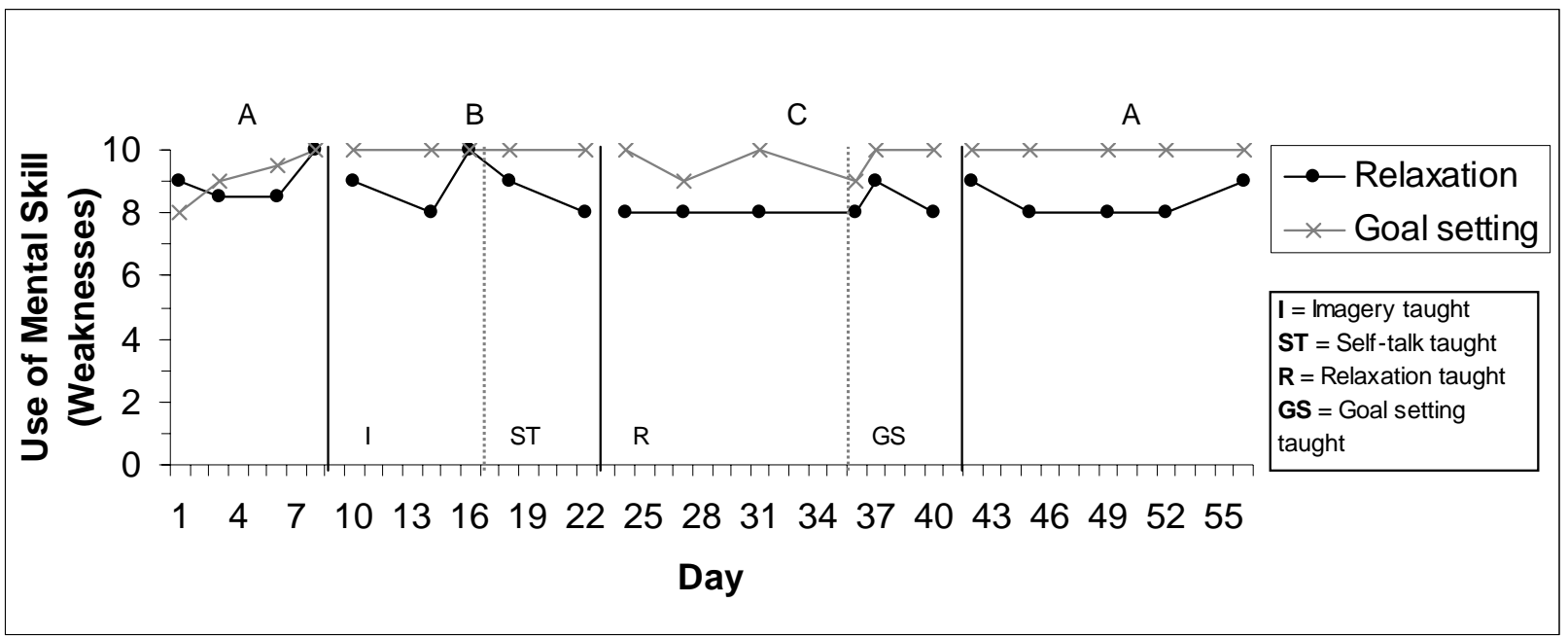

Figure 6b. Perceived effectiveness of mental skills (weaknesses) on rehabilitation for P3.

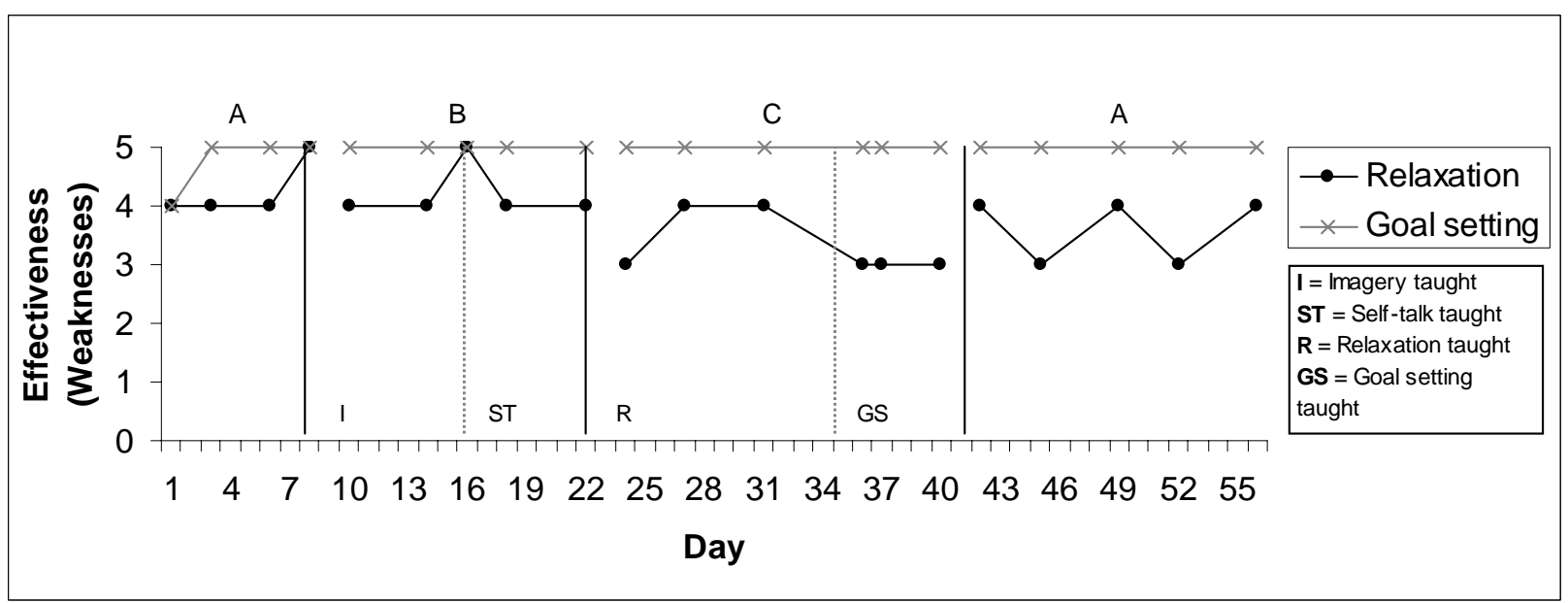


Figure 7a. Self-reported use of mental skills (strengths) for P4.

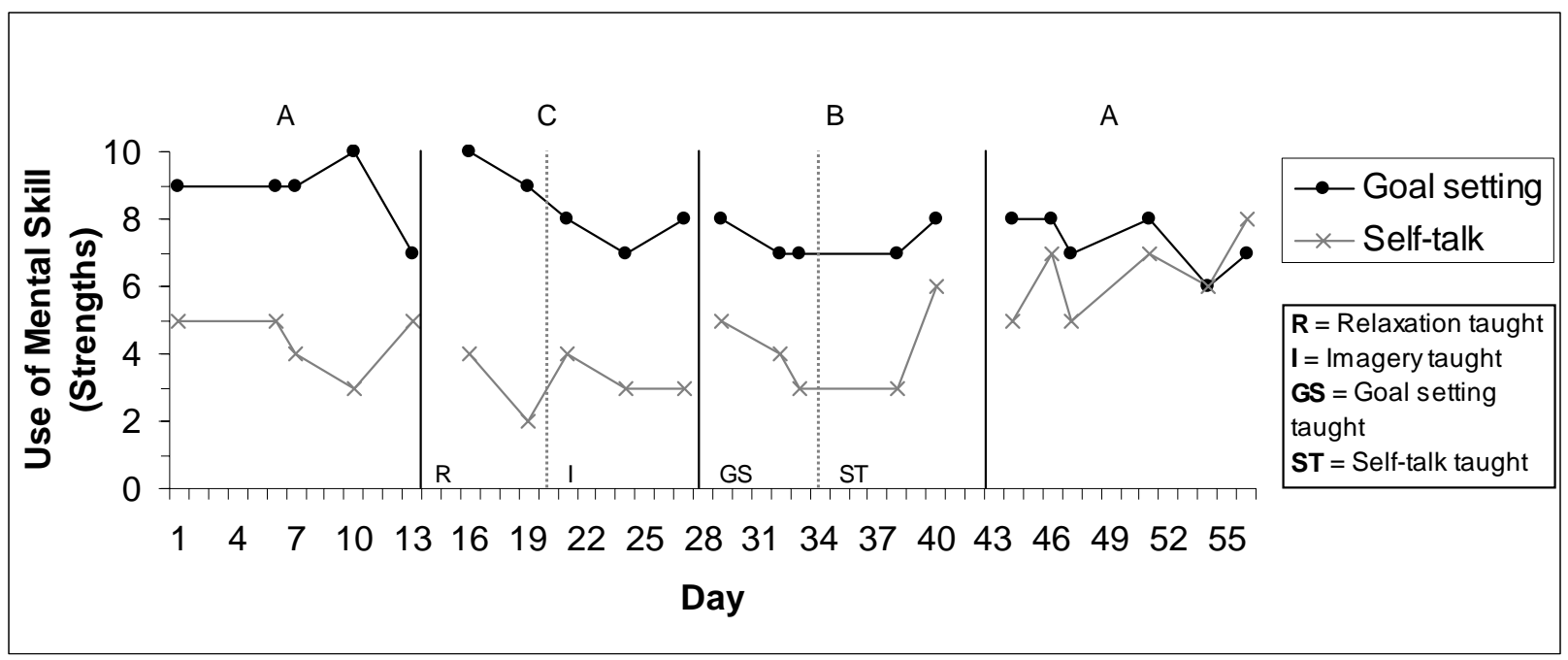

Figure $7 b$. Perceived effectiveness of mental skills (strengths) on rehabilitation for P4.

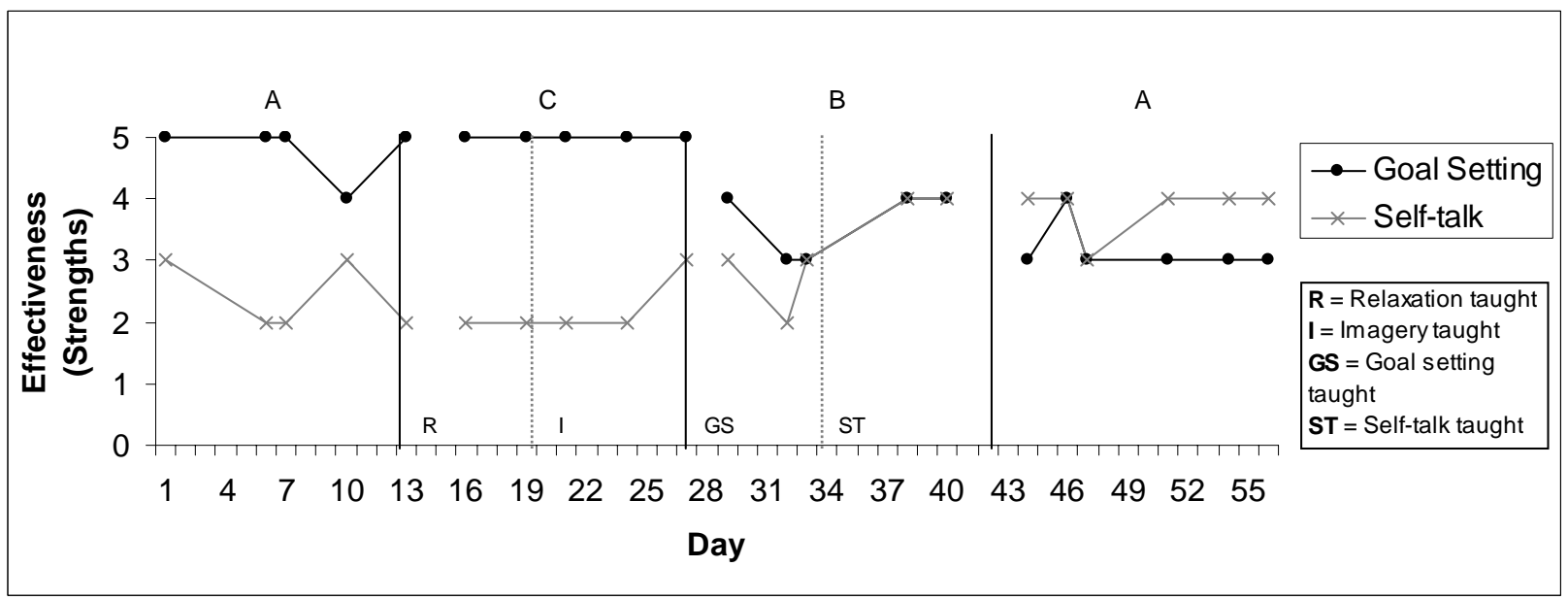


Figure 8a. Self-reported use of mental skills (weaknesses) for P4.

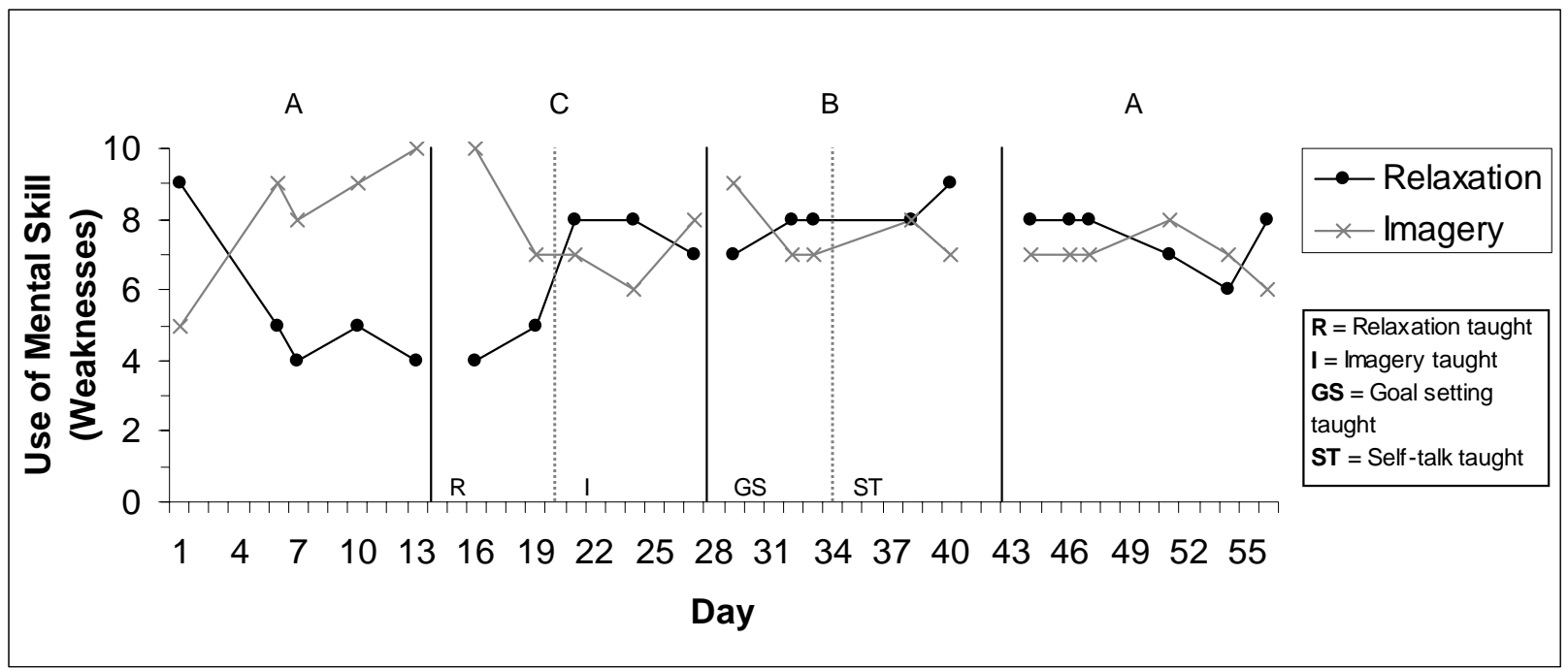

Figure 8b. Perceived effectiveness of mental skills (weaknesses) on rehabilitation for P4.

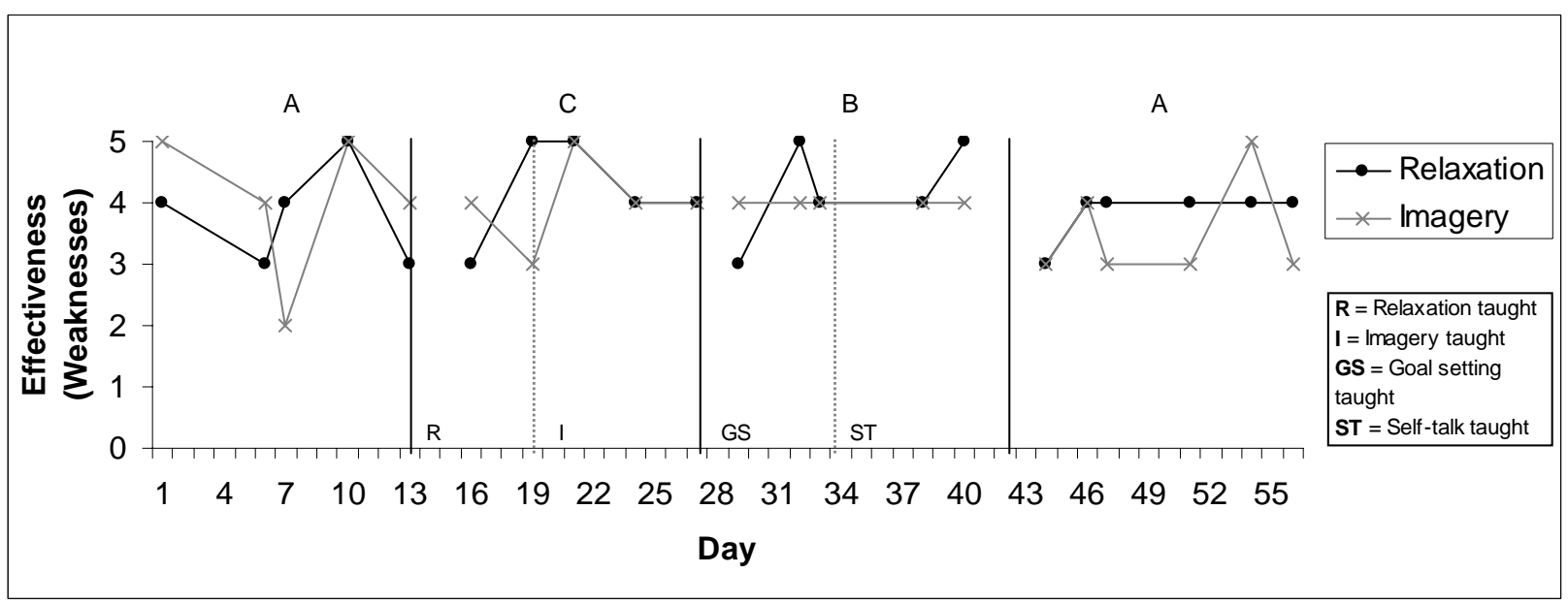


Figure 9a. Self-reported use of mental skills (strengths) for P5.

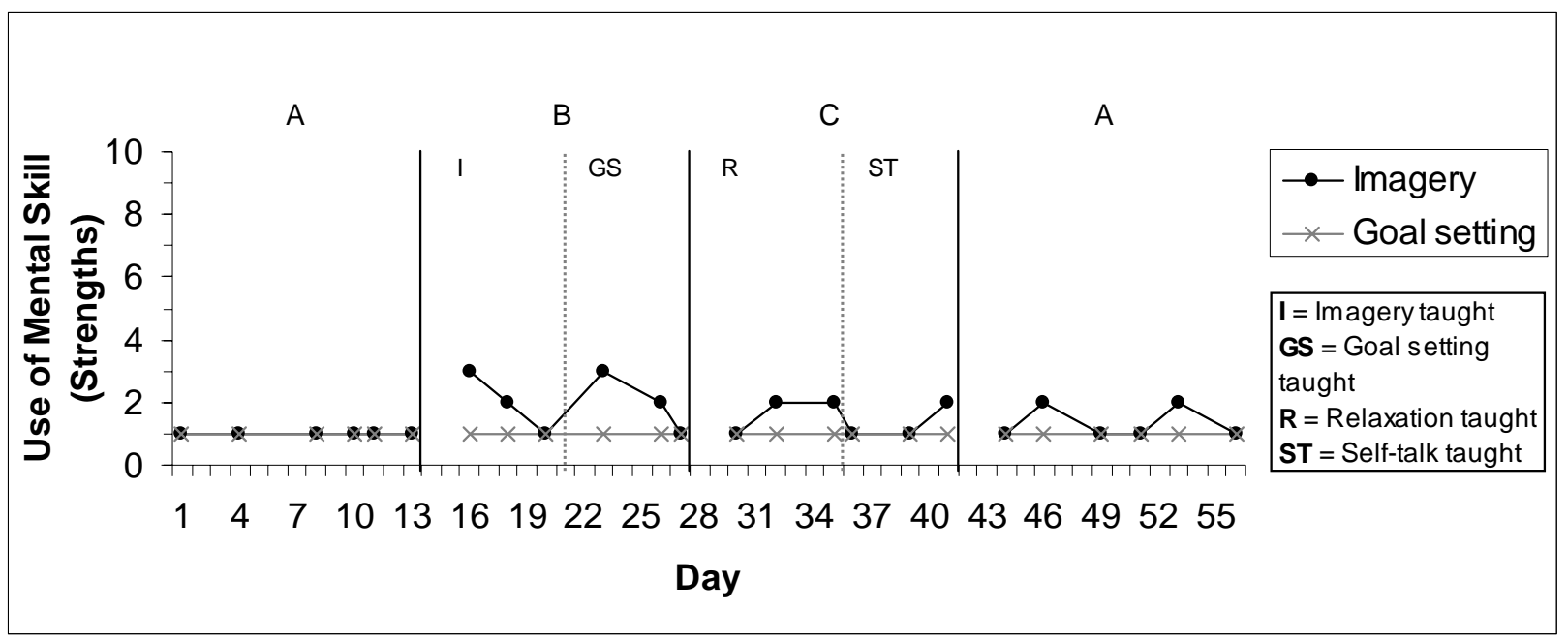

Figure 9b. Perceived effectiveness of mental skills (strengths) on rehabilitation for P5.

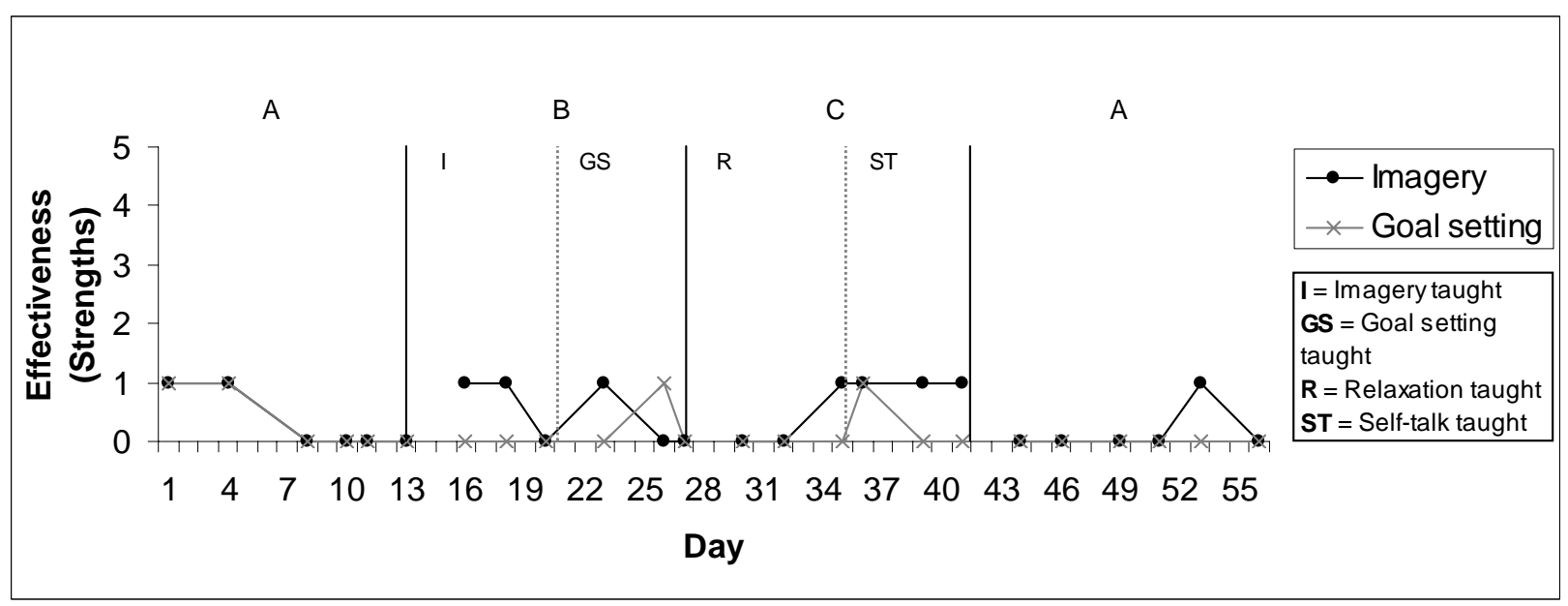


Figure 10a. Self-reported use of mental skills (weaknesses) for P5.

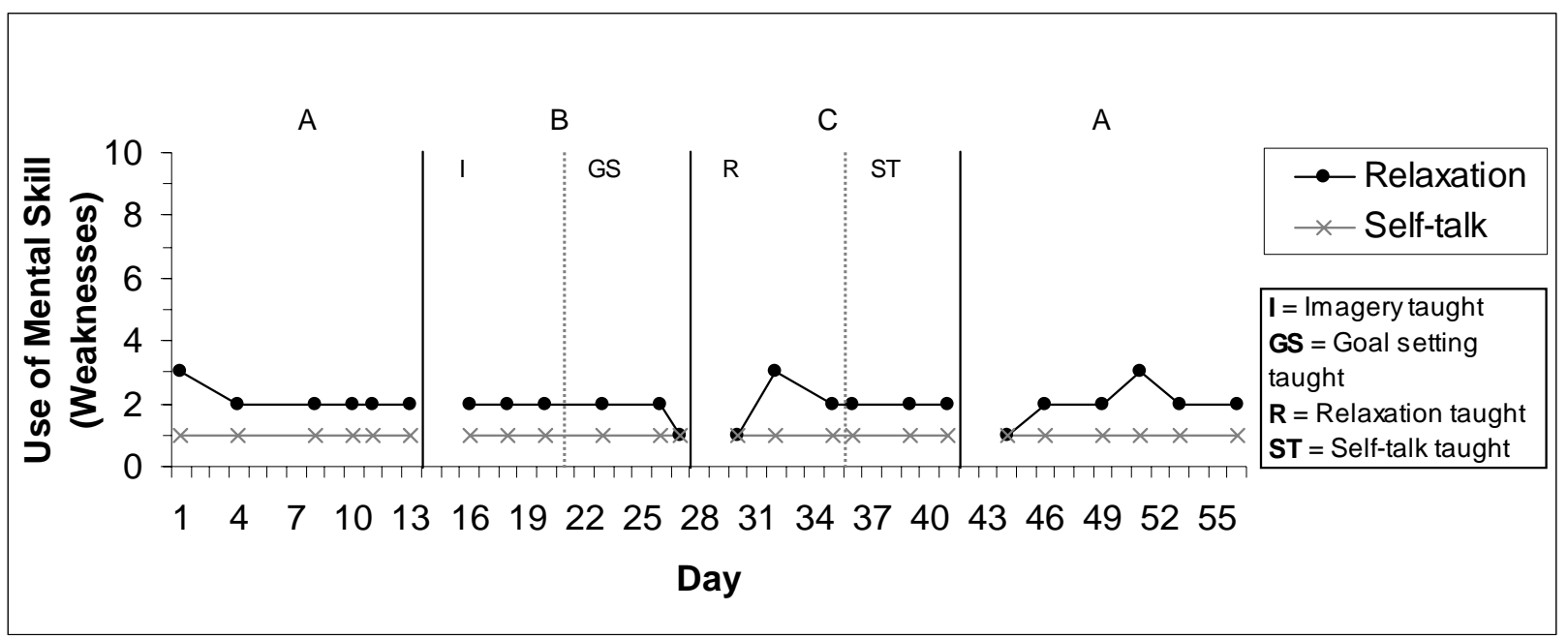

Figure 10b. Perceived effectiveness of mental skills (weaknesses) on rehabilitation for P5.

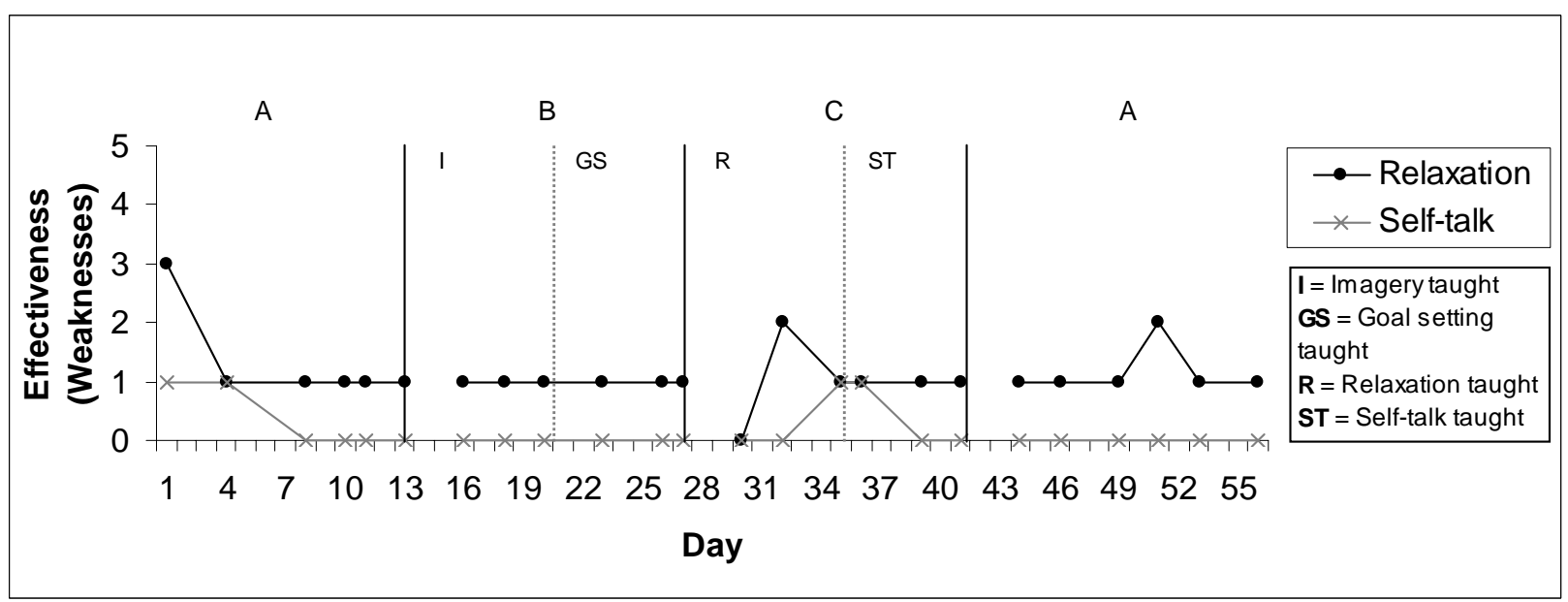


Figure 11a. Self-reported use of mental skills (strengths) for P6.

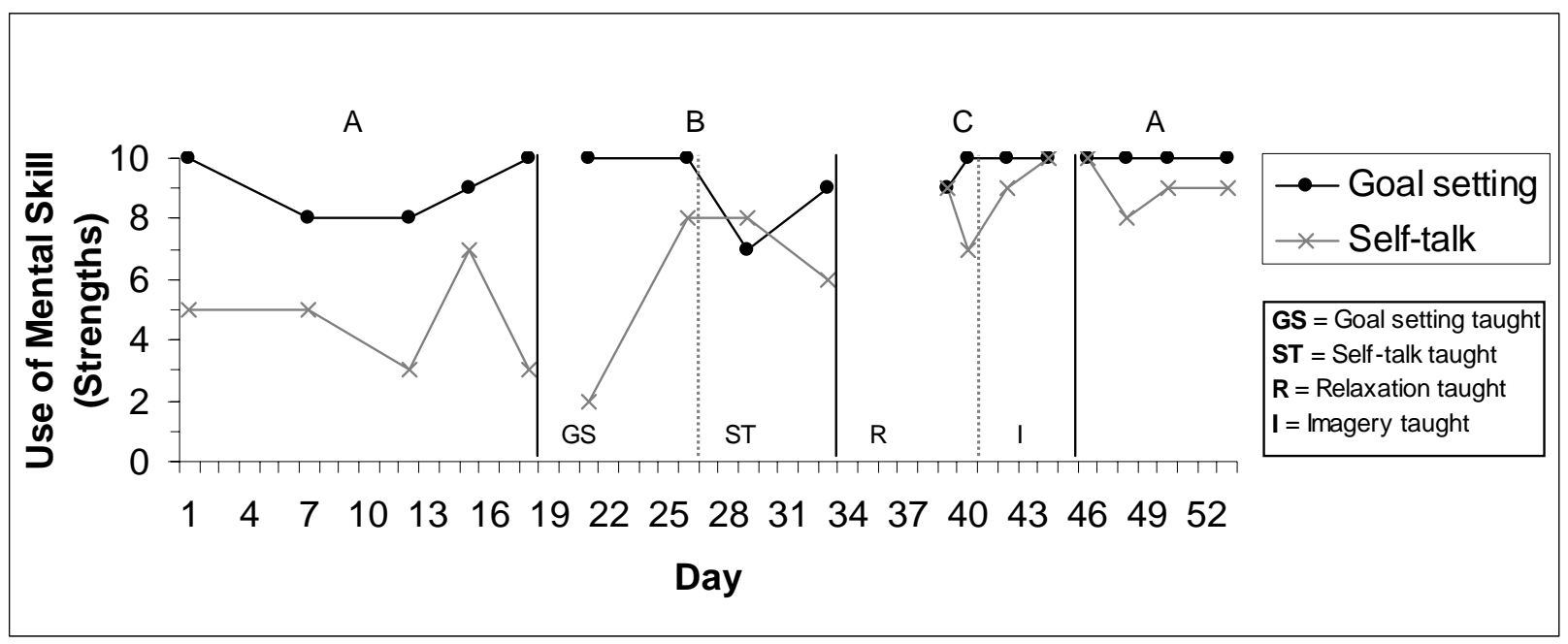

Figure 11b. Perceived effectiveness of mental skills (strengths) on rehabilitation for P6.

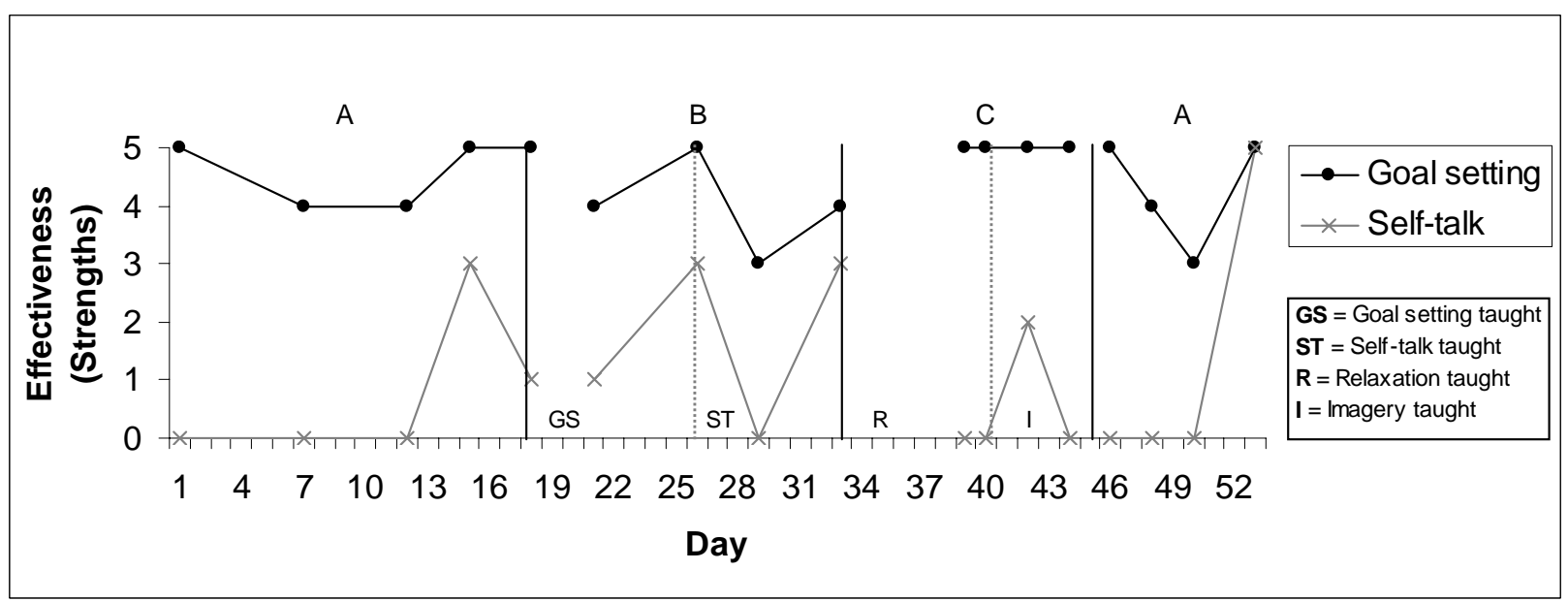


Figure 12a. Self-reported use of mental skills (weaknesses) for P6.

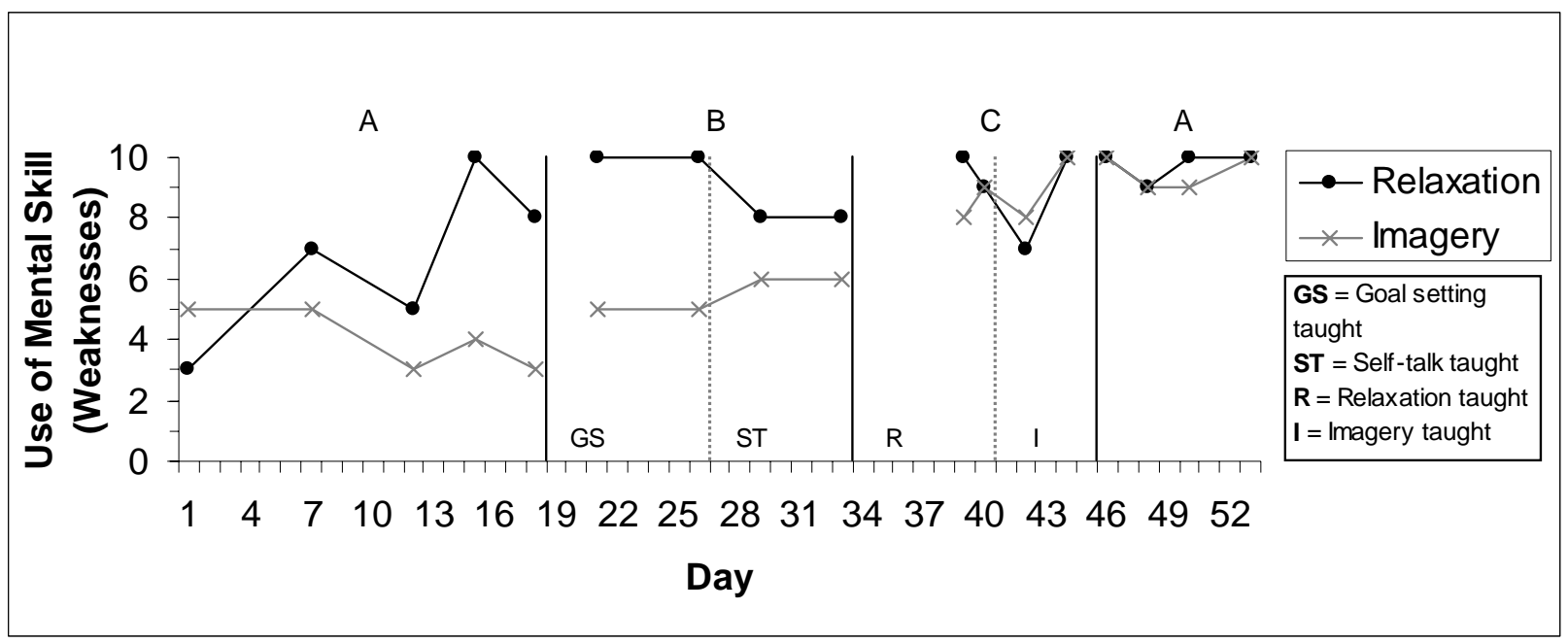

Figure 12b. Perceived effectiveness of mental skills (weaknesses) on rehabilitation for P6.

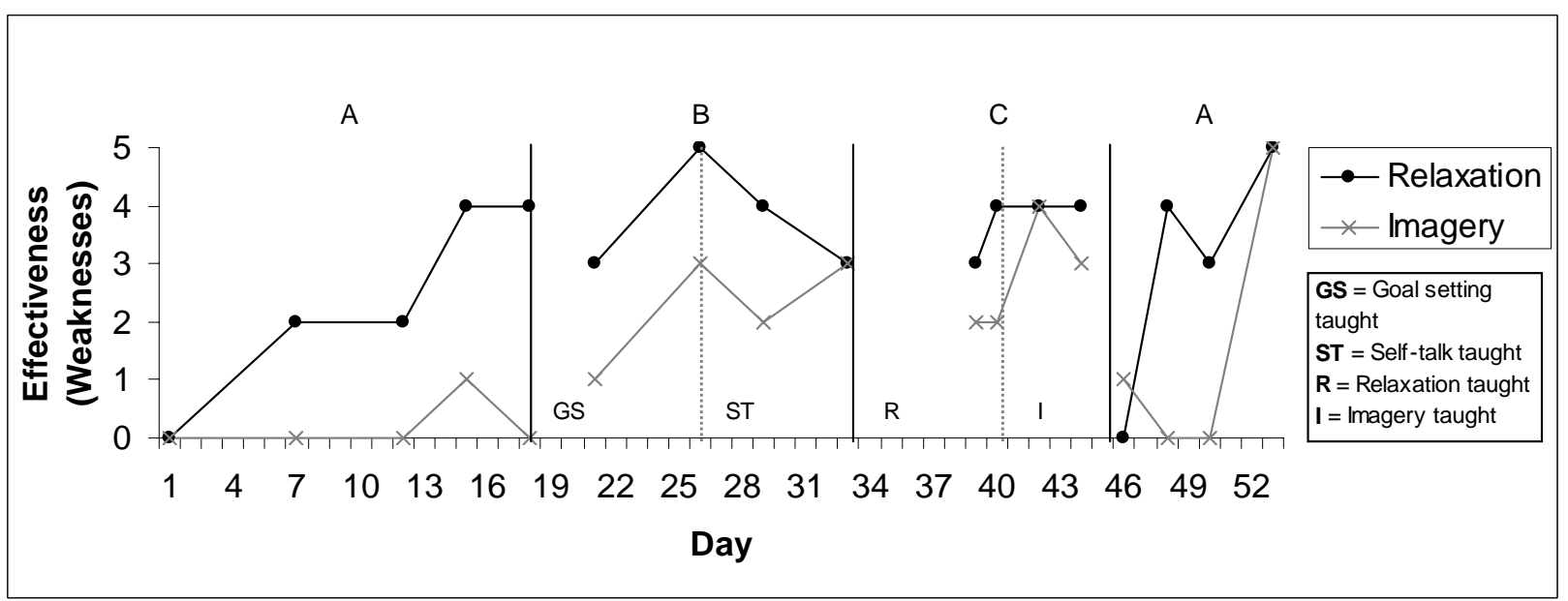


Figure 13. Self-efficacy for rehabilitation for P1.

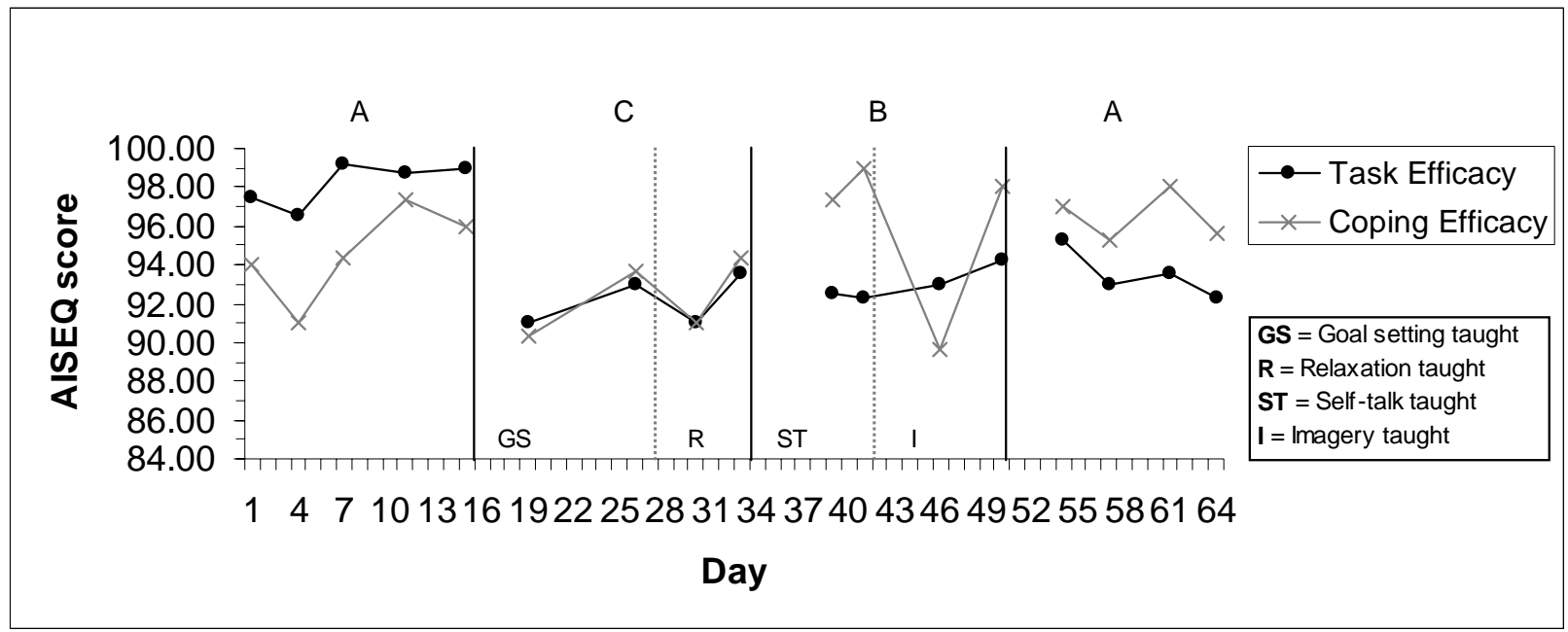

Figure 14. Self-efficacy for rehabilitation for P2.

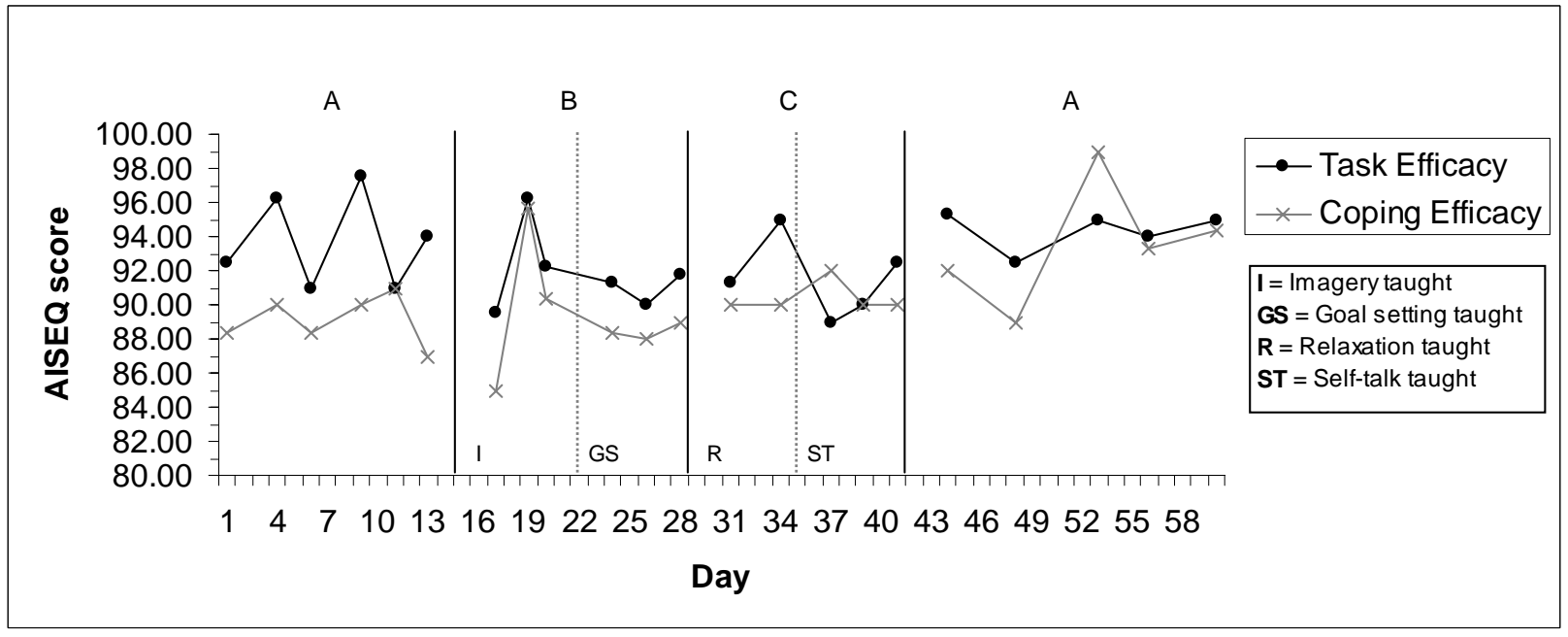

Figure 15. Self-efficacy for rehabilitation for P3.

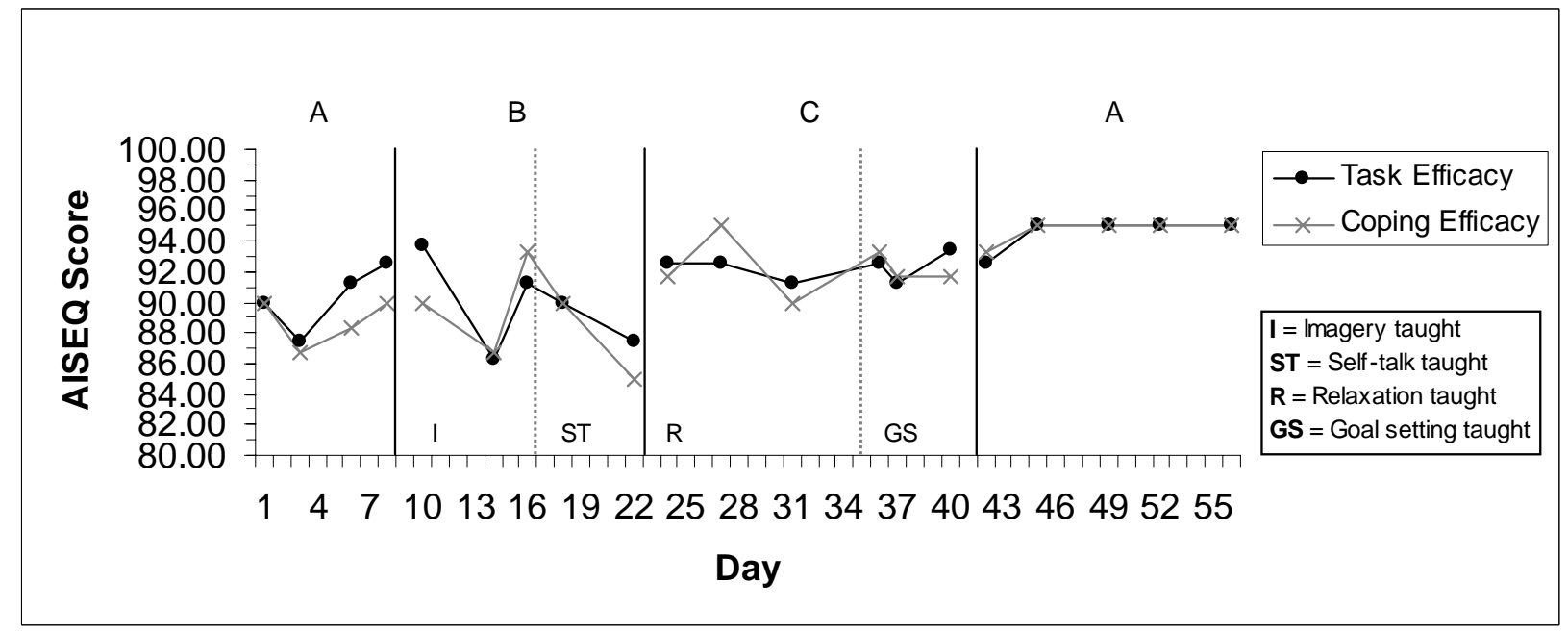


Figure 16. Self-efficacy for rehabilitation for P4.

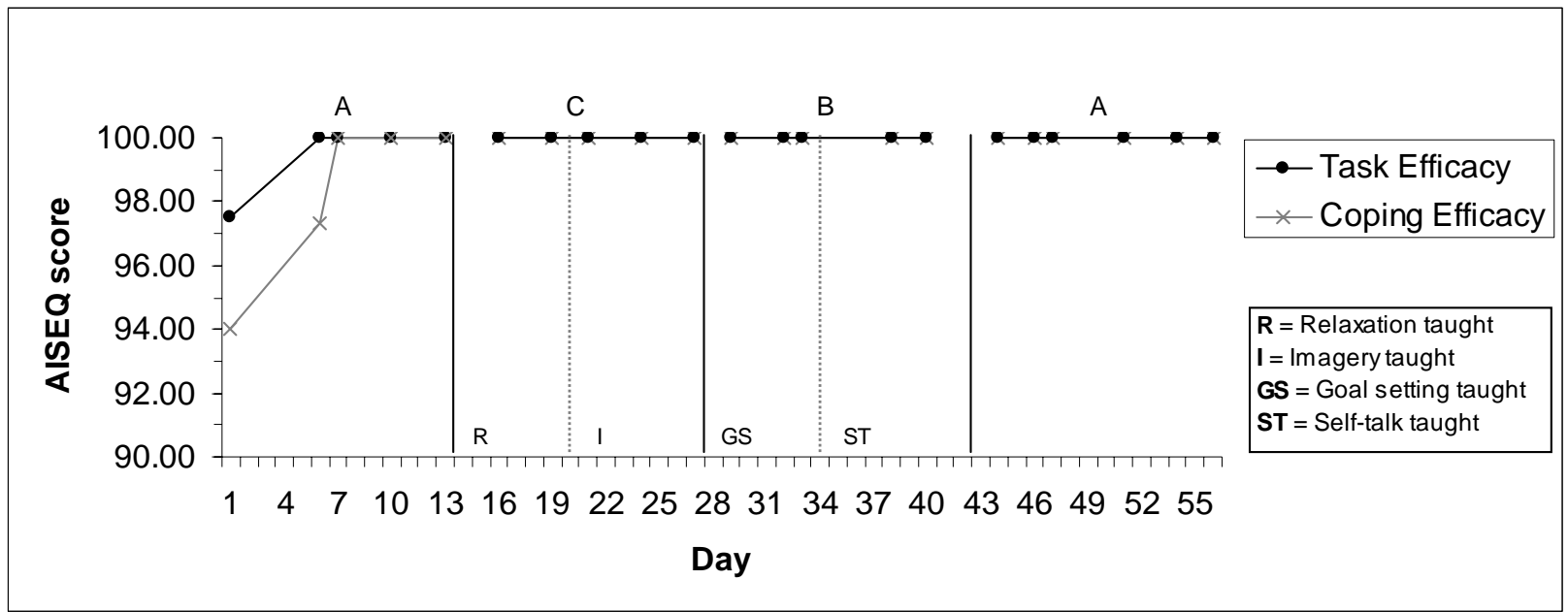

Figure 17. Self-efficacy for rehabilitation for P5.

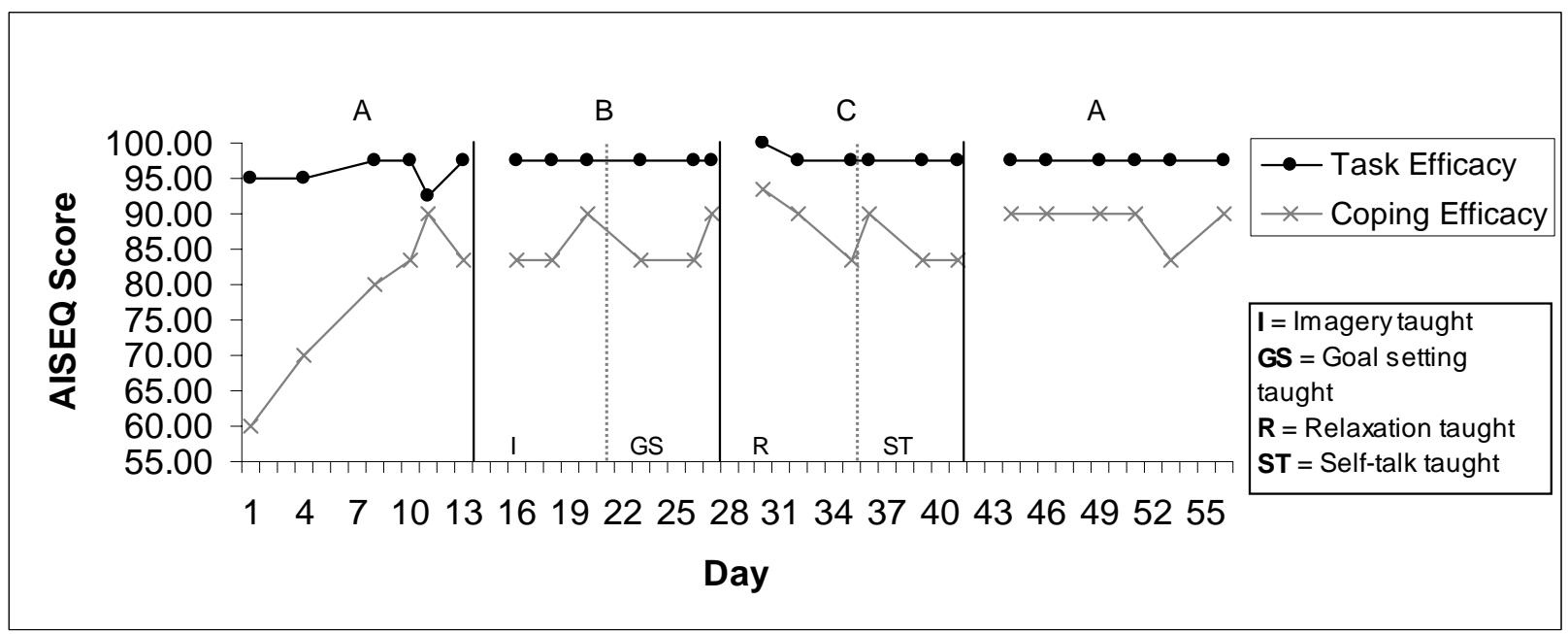

Figure 18. Self-efficacy for rehabilitation for P6.

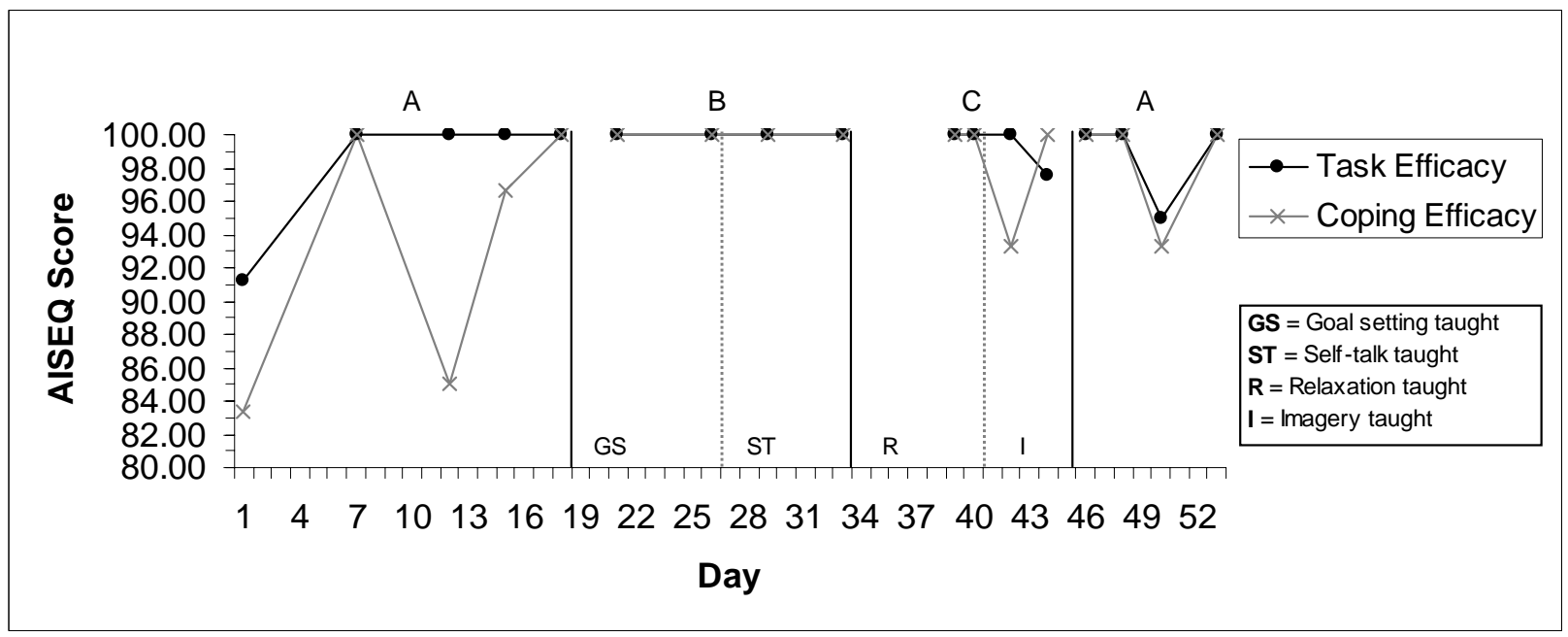


Figure 19. Self-rated adherence and attitude for P1.

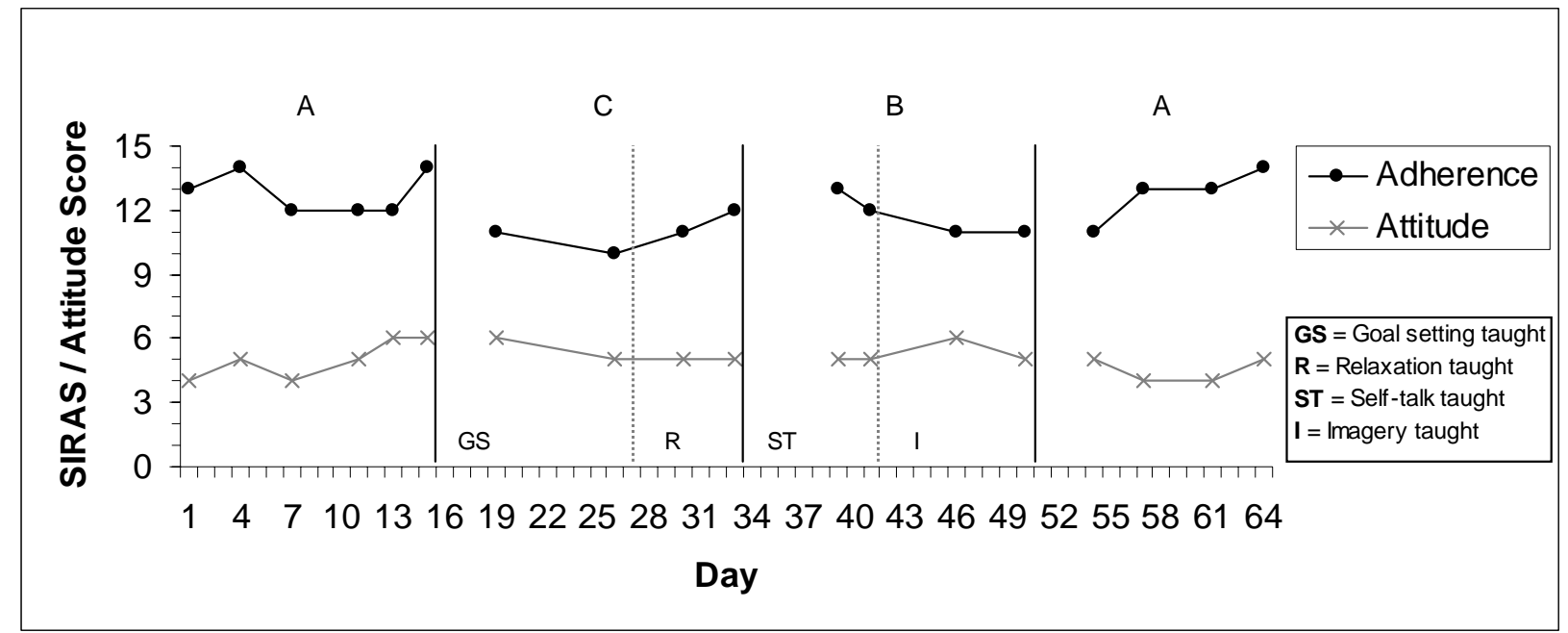

Figure 20. Athletic Trainer-rated data for P3.

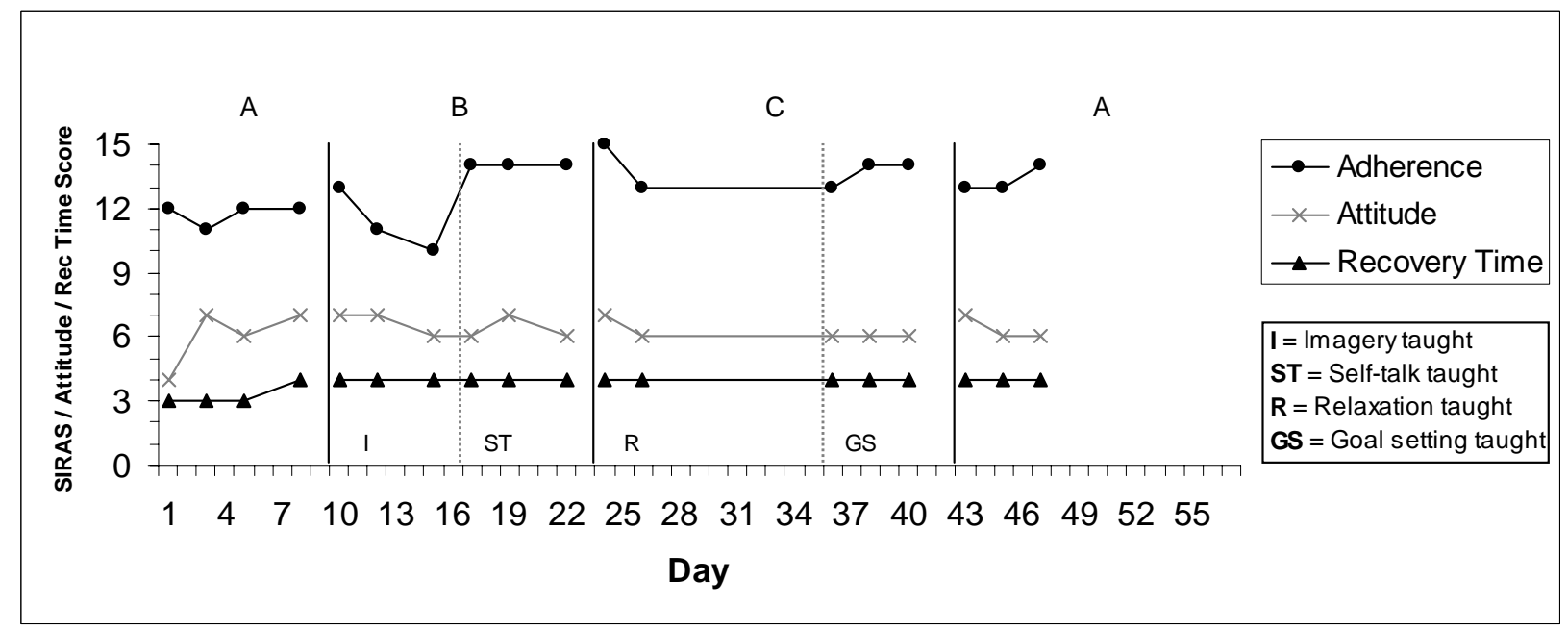

Figure 21. Self-rated adherence and attitude for P2.

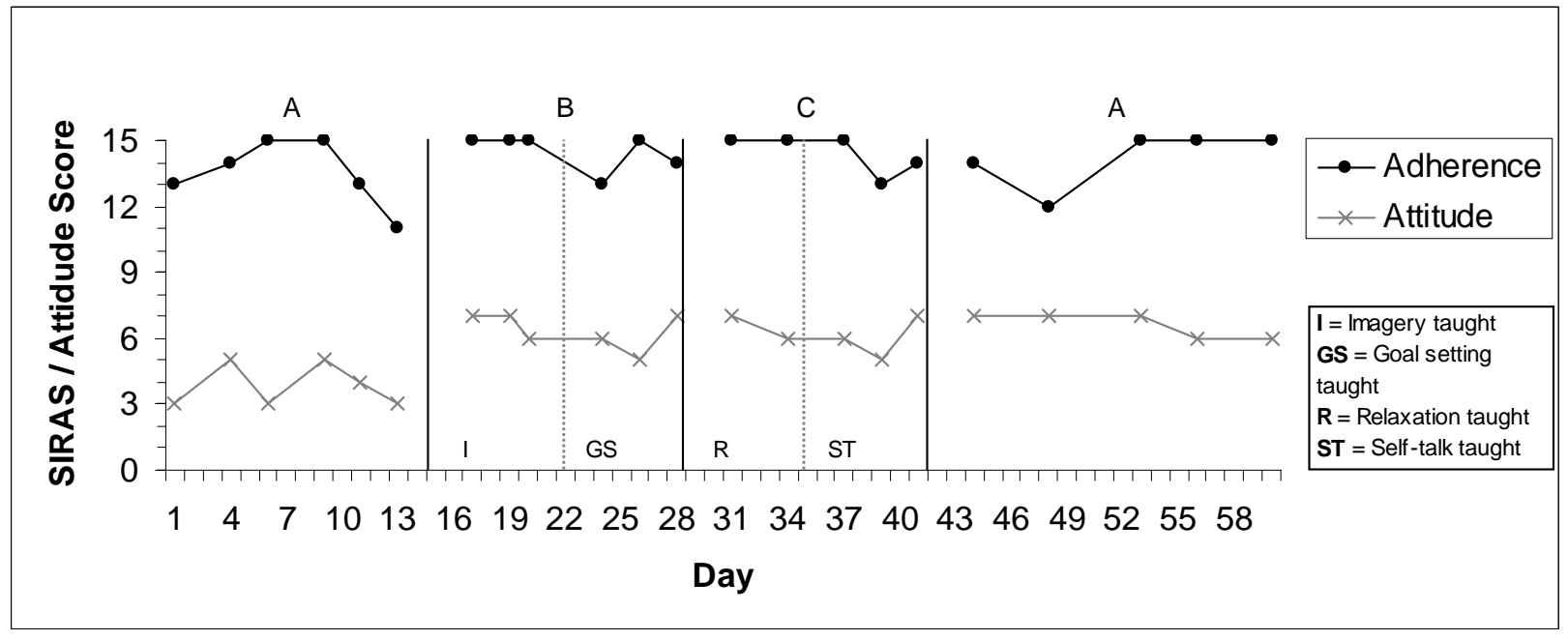


Figure 22. Athletic Trainer-rated data for P1.

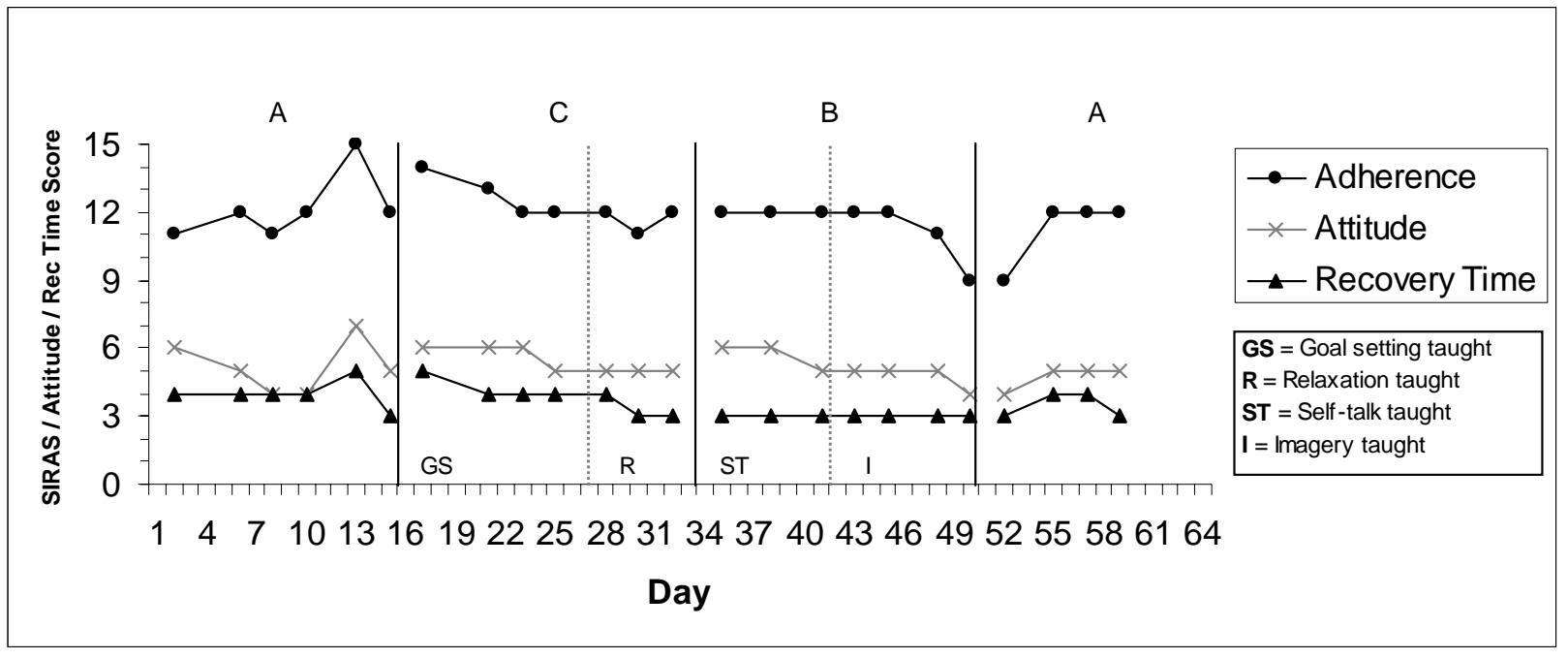

Figure 23. Self-rated adherence and attitude for P6.

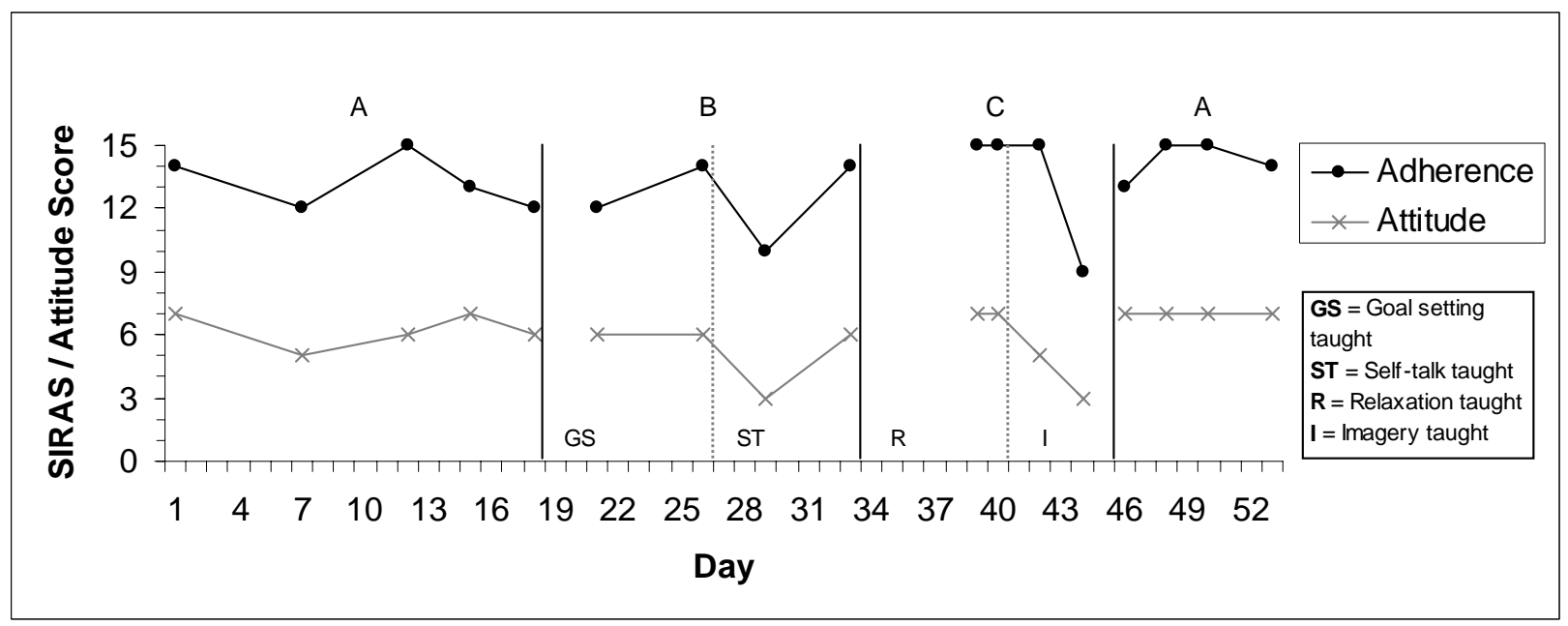




\section{APPENDIX A: Review of the Literature}

Introduction

Although only a minority of injured athletes (5-24\%) appear to experience clinically meaningful levels of psychological distress (Brewer \& Cornelius, 2003), it is clear that responses to injury are not just physical, but psychological as well (e.g., Brewer, Anderson, \& Van Raalte, 2002; Wiese-Bjornstal, Smith, Shaffer, \& Morrey, 1998). Since athletes, coaches, athletic trainers, and sports medicine personnel are all interested in the most efficient recovery for injured athletes, rehabilitation from injury should include both physical and psychological interventions. While numerous researchers have advocated the use of psychological strategies in injury rehabilitation, methodologically sound, controlled, empirical studies of actual psychological interventions done with injured athletes are scarce (Cupal, 1998). In order to examine the psychological responses to injury and psychological factors which may affect rehabilitation, the literature review will be presented in the following four sections: (1) models of psychological responses to sport injury; (2) psychological factors associated with injury rehabilitation outcomes, specifically adherence and recovery time from injury; (3) suggestions for implementing psychological interventions for sport injury rehabilitation; and (4) empirical research on psychological interventions for injury rehabilitation, including perceptions of psychological interventions for injured athletes, athlete self-reported use of coping/mental skills during injury recovery, and intervention studies of mental skills with injured participants. A summary will be provided at the end of the review.

\section{Models of Psychological Responses to Sport Injury}

While an injury is physically debilitating and requires physical rehabilitation, athletes also experience psychological responses to injury. Emotional disturbance is greater in athletes 
with injuries than athletes without injuries. Approximately 5 to 24\% of athletes with injuries experience clinically meaningful levels of psychological distress. For the most part, emotional responses of athletes with injuries tend to become more adaptive over time (see Brewer \& Cornelius, 2003, for a review of psychological factors in sports injury rehabilitation).

Several models have been proposed to explain the psychological response to injury. Since an injury can be considered a loss of an aspect of the self, the Kubler-Ross (1969) stage model of grief and loss has often been used to explain the psychological response to injury. Kubler-Ross proposed that in response to a loss, one would sequentially experience the stages of denial, anger, bargaining, depression, and acceptance. However, support for this model has not been strong in the sport injury literature (Brewer, 1994; Evans \& Hardy, 1995). Brewer pointed out that stage models ignore important aspects of the injury response including perceptions of the injury and situational circumstances surrounding the injury (e.g., timing and severity of injury, social support). Also, stage models do not take into account individual variability in response to injury. A second model of psychological response to injury is the integrated model of psychological response to the sport injury and rehabilitation process (Wiese-Bjornstal, Smith, Shaffer, \& Morrey, 1998). Pre-injury variables such as stress, personality, and coping skills interact with post-injury variables to determine athletes’ cognitive, emotional, and behavioral responses to injury. Albinson and Petrie (2003) reported some support for the interaction of preand post-injury variables in that negative life stress (prior to injury) was related to mood disturbance the day following injury for collegiate football players $(n=18)$. Although not statistically significant, a large effect was found for the relationship between negative life-stress (preinjury) and secondary appraisal (ability to cope with injury) at four days post-injury $(n=7)$. Those who reported more negative stress prior to injury perceived more coping difficulties after 
injury. A non-significant large effect was also found between dispositional optimism and primary appraisal (perception of injury as stressful) at four days post-injury. Those with higher reported levels of optimism perceived the injury as less stressful. The small sample size likely contributed to the results being nonsignificant.

According to the integrated model (Wiese-Bjornstal et al., 1998), following injury, an athlete appraises the injury (cognitive appraisal) in terms of what the injury means to him/her. This cognitive appraisal also includes one’s self-efficacy for coping with the injury and recovering. Cognitive appraisal of the injury affects emotional (e.g., fear, tension, anger, depression, frustration, attitude) and behavioral (e.g., adherence, effort, use of psychological skills training, use of social support) responses to injury. According to this model, cognitive appraisals, emotional responses, and behavioral responses reciprocally influence each other. In addition, personal (e.g., injury type and severity, personality, athletic identity) and situational factors (e.g., sport, social support, rehabilitation environment) are always interacting with the response process, exerting their effects throughout. Albinson and Petrie (2003) examined the relationship between cognitive appraisals and emotional responses following injury as well as cognitive appraisals and choice of coping strategy. They found that athletes who perceived their injuries as stressful and difficult to cope with had elevations in mood disturbance, particularly one month after injury. Daly, Brewer, Van Raalte, Petitpas, and Sklar (1995) also found that negative cognitive appraisals were related to total mood disturbance following injury. Athletes with negative cognitive appraisals were more likely to use active behavioral and avoidance coping and less likely to use active cognitive coping to deal with their injuries (Albinson \& Petrie). 
Another model of psychological response to sport injury is the biopsychosocial approach (Brewer, Anderson, \& Van Raalte, 2002). This model incorporates biological, social, medical, and psychological factors. The model has seven interacting components: injury characteristics, sociodemographic factors, biological factors (e.g., nutrition, sleep, immune function), psychological factors (e.g., personality, affect, cognition), social/contextual factors (e.g., social network, life stress, rehabilitation environment), intermediate biopsychological outcomes (e.g., range of motion, strength, pain, rate of recovery), and sport injury rehabilitation outcomes (e.g., treatment satisfaction, readiness to return to sport). While both the integrated and biopsychosocial models have some practical merit as factors to consider while conceptualizing an athlete’s response to injury, these models have not been tested as a whole and are based on little actual data.

A model of psychological response to injury that comes from the qualitative literature is the Restoration of Self Model (Mainwaring, 1999). Mainwaring used the grounded theory method with a combined cross-sectional and longitudinal (over a 12 month period) sample of athletes to conceptualize this model. The overarching concept is that after a severe injury, athletes strive to overcome their disability and restore their sense of self to what it was preinjury. Using qualitative analyses, Mainwaring found that the response to injury involves the interaction of person and situational variables and includes three major domains: (1) physical, (2) psychological (including cognitive and affective dimensions), and (3) social. Although generally optimistic about recovery, athletes had negative affect throughout the rehabilitation process with positive affect interspersed when rehabilitation gains were made. Participants were found to use more active task-focused coping skills as opposed to passive emotion-focused coping. Participants experienced social consequences of their injury, including isolation from their team. 
It is important to note that only participants with severe knee injuries were used in this study, therefore the model may only apply to that type of injury. This model has yet to be tested by other researchers.

Psychological Factors Associated with Injury Rehabilitation Outcomes

Two injury rehabilitation outcomes of interest to sport professionals are: (1) adherence to the rehabilitation protocol and (2) recovery time from injury. Several researchers (e.g., Brewer et al., 2004; Pizzari, Taylor, McBurney, \& Feller, 2005) have found a positive relationship between adherence and physical outcomes in rehabilitation, thereby giving support to the importance of adherence to the successful recovery of an injured athlete. However, the level of adherence necessary to improve rehabilitation outcomes has not been defined; in other words, $100 \%$ adherence may not necessarily be the goal to obtain. Another outcome variable in sport injury rehabilitation is recovery time from the injury. Quinn and Fallon (2000) argued that duration of recovery is one of the most important and meaningful factors in injury recovery for athletes, teams, and coaches; returning to full athletic participation as quickly as possible is a priority. However, few studies have explored this outcome measure in the sport psychology literature. Psychological factors that affect adherence and recovery time from injury will be discussed below.

Factors Affecting Adherence

Levy, Polman, Clough, and McNaughton (2006) provided a review of various theories in relation to adherence to sport injury rehabilitation (e.g., Personal Investment Theory, Protection Motivation Theory, Attribution Theory, Cognitive Appraisal Models, Health Action Process Approach, and Theory of Planned Behavior). Many of these theories or frameworks include selfefficacy as a component and numerous studies have found self-efficacy to be related to 
adherence. Self-efficacy is the belief in one's ability to perform a certain task (Bandura, 1977). Self-efficacy Theory incorporates three components: (1) the expectation that one can perform a specific behavior; (2) the expectation that engagement in such behavior will have positive outcomes; and (3) the value of those outcomes to the individual. Self-efficacy can come from several different sources of information including: performance accomplishments, vicarious experience, verbal persuasion, and physiological states (Bandura, 1977). Since injury alters one’s physiological state, it is likely that injury will affect one's self-efficacy in certain situations. Milne, Hall, and Forwell (2005) found that both task self-efficacy (i.e., belief in one’s ability to perform one's rehabilitation exercises) and coping self-efficacy (i.e., belief that one can perform one’s exercises despite obstacles) were found to predict aspects of rehabilitation adherence (quality, frequency, and duration of exercises). An implication of these results is that practitioners should work to build athletes' self-efficacy during the rehabilitation process to improve adherence. While several researchers have found that one’s expectancy to be successful in rehabilitation is related to adherence to sport injury rehabilitation (e.g., Brewer et al., 2003; Milne, Hall, \& Forwell, 2005; Taylor \& May, 1996), Taylor and May (1996) also found support that the value one places on being fully recovered from injury is related to adherence, lending more support to Self-efficacy Theory. Evans, Harding, and Flemming (2000) also found qualitative support for the effect of outcome expectancies on adherence.

In Protection Motivation Theory (PMT; Maddux \& Rodgers, 1983), one’s motivation to protect oneself from a health threat is thought to be influenced by two threat appraisals and two coping appraisals. The threat appraisals consist of: (1) perceived susceptibility to the threat and (2) perceived severity of the threat. The coping appraisals consist of: (1) self-efficacy (to perform a required health behavior) and (2) treatment efficacy (belief in the treatment to overcome a 
threat). Taylor and May (1996) developed and used the Sport Injury Rehabilitation Beliefs Survey (SIRBS) with 62 injured student-athletes to assess how the four components of PMT related to adherence to the rehabilitation protocol and adherence to rest, using both a physiotherapist estimate and self-report of compliance. Their findings provided some support for the role of threat appraisal as a motivating process in the decision to adhere to the prescribed sport injury rehabilitation protocol. Severity of injury was the strongest threat appraisal predictor of adherence to the rehabilitation protocol. In contrast, susceptibility was the strongest predictor of adherence to rest. The findings were also partially supportive of the role of coping appraisal in motivating athletes to adhere to rehabilitation programs. Those with stronger beliefs in their ability to complete the prescribed rehabilitation modalities (self-efficacy), and those with greater expectancy in the benefits of such action (treatment efficacy), were more likely to be adherent. However, the findings did not consistently reveal (between all of the different measures of compliance) that athletes with greater adherence initially reported higher perceptions of selfefficacy or treatment efficacy than less adherent athletes. The findings from this study suggest that practitioners should at least raise athletes' awareness of the potential health risk associated with non-adherence to the rehabilitation regimen. They should also use strategies to increase athletes' self-efficacy and perceptions of the benefits of adhering to the prescribed protocol.

A more recent study by Brewer et al. (2003) also examined the role of Protection Motivation Theory variables in adherence to sport injury rehabilitation programs. Unlike Taylor and May’s study (1996), this study used athletes $(N=85)$ rehabbing from the same injury (ACL reconstruction) and assessed adherence to both home-based and clinic-based adherence. Their results provided support for PMT as a useful framework for understanding adherence to homebased as well as clinic-based sport injury rehabilitation activities. The coping appraisal 
components (i.e., treatment efficacy and self-efficacy) were more strongly associated with adherence than the threat appraisal components (i.e., susceptibility and severity). Severity was associated with none of the adherence measures. The authors cautioned that their findings were correlational, and note that to fully evaluate the causal contribution of threat and coping appraisals to sport injury rehabilitation adherence, experimental research is needed in which protection motivation theory parameters are manipulated and the effects on adherence are evaluated. Their findings did suggest that increasing one’s self-efficacy and perceived treatment efficacy should improve adherence in injury rehabilitation.

The Health Action Process Approach (HAPA; Schwarzer, 1992) is another model that has been examined in relation to adherence to sport injury rehabilitation. This approach posits that the adoption, initiation, and maintenance of a health behavior involves two phases: (1) a volitional (planning) phase and (2) a motivational (including intention) phase. Self-efficacy is hypothesized to influence both of these phases. In examining the adoption and maintenance of physical exercise with orthopedic rehabilitation patients $(N=509)$, Lippke, Ziegelmann, and Schwarzer (2004) found that self-efficacy and outcome expectations were direct precursors of planning and intentions to exercise. Therefore, increasing one’s self-efficacy and improving outcome expectancies can affect adoption, initiation, and maintenance of health behaviors, such as rehabilitation from an injury. Although the above theories (Self-efficacy Theory, Protection Motivation Theory, and the Health Action Process Approach) incorporate different constructs, all of the research examining these theories in relation to adherence to sport injury rehabilitation has supported the notion that one's belief in one's ability to successfully recover (self-efficacy) influences adherence. 
Levy, Polman, and Clough (2008) examined predictors of rehabilitation intention and behavior from their proposed model, called the Adapted Planned Behavior Model (APBM; Levy, Polman, \& Clough, 2005, as cited in Levy et al., 2008). The APBM is based on the Theory of Planned Behavior (TPB; Ajzen, 1991, as cited in Levy et al., 2008). The model posits that primary factors (i.e., threat appraisals, goal orientation, attitude) predict intention to engage in rehabilitation, which then leads to adherence. Secondary factors (i.e., coping ability, treatment efficacy, social support) would predict adherence. Self-efficacy and self-motivation would both indirectly (via intention) and directly predict adherence. Finally, as rehabilitation behavior is done on a consistent basis, it becomes "habit" which is also postulated to predict adherence in the APBM.

In examining athletes with a tendonitis-related overuse injury $(N=70)$, Levy, Polman, and Clough (2008) found that the primary factors of perceived severity/susceptibility, task orientation, and positive attitude significantly predicted intention to engage in rehabilitation, accounting for $53 \%$ of the variance. Contrary to the proposed model, self-efficacy and selfmotivation did not predict intention, but did predict clinic-based adherence and attendance. The secondary factors of coping ability, treatment efficacy, habit, and several types of social support (i.e., task appreciation by physiotherapist and emotional support by friends) predicted clinic based adherence, accounting for $68 \%$ of the variance. One weakness of the study was that the secondary factors were based on retrospective reports by the participants. A factor to consider is that the majority of participants were recreational athletes and all with the same injury; thus, the results may not generalize to athletes at more competitive levels and with other types of injuries.

The above research studies incorporated a deductive approach by examining adherence in relation to an established theory. Niven (2007) used an inductive qualitative method to explore 
adherence to sport injury rehabilitation by interviewing experienced sports physiotherapists $(N=$ 9). Niven investigated four areas: (1) incidence and importance of adherence in practice; (2) factors that influence good adherence to rehabilitation; (3) factors that influence poor adherence to rehabilitation; and (4) strategies used to improve adherence. Results indicated that most athletes do comply with rehabilitation prescriptions and that adherence is important for recovery.

Several factors influenced good adherence including: a professional approach to rehabilitation, adaptable, educated, confident, used to pain, enjoys physical activity, and motivated and driven to succeed. A positive attitude towards rehabilitation as well as an understanding of the injury and rehabilitation was also deemed important for adherence. Good support structures (i.e., social support, trust in physiotherapist and rehabilitation) and a facilitative environment were situational factors that were found to be important for adherence. Previous experience with injury was found to be a facilitative factor of adherence because the athlete knew what they needed to do to get better. Factors that influenced poor adherence to rehabilitation included: unprofessional approach, unmotivated, less intelligent, negative emotions, maladaptive thoughts (i.e., lack of confidence, lack of understanding of injury and rehabilitation, negative attitude), lack of social support, environmental constraints, skepticism about rehabilitation, pressure from others, and poor past experiences in rehabilitation. The strategy that physiotherapists identified as most important to facilitating adherence was goal setting and monitoring progress (Niven, 2007).

Injured athletes’ self-reported use of various psychological skills have been found to be related to injury rehabilitation adherence. For instance, Scherzer et al. (2001) examined athletes’ $(N=54)$ self-reported use of several psychological skills (i.e., goal setting, positive self-talk, and imagery) in injury rehabilitation in relation to adherence. The researchers found that goal setting 
was positively related to home exercise completion and trainer adherence ratings. Also, positive self-talk was positively associated with home exercise completion. Other researchers have looked at self-reported use of imagery and the impact on injury outcome variables. For example, Milne, Hall, and Forwell (2005) found that cognitive imagery (i.e., mental rehearsal of rehabilitation exercises) was a significant predictor of task self-efficacy (i.e., belief in one’s ability to perform rehabilitation exercises). However, the amount of variance accounted for in self-efficacy by imagery was small (1.8\%). The researchers also found that both task and coping self-efficacy predicted various aspects of adherence; therefore, there may be an indirect relationship between imagery use and adherence, with self-efficacy as a mediating variable. Research exploring how these psychological skills impact injury recovery will be discussed in greater depth below (see section entitled, Empirical Research on Psychological Interventions for Injury Rehabilitation).

\section{Factors Affecting Recovery Time from Injury}

Quinn and Fallon (2000) examined predictors of recovery time from athletic injuries $(N=$ 136) at four phases of rehabilitation: (1) immediately after the injury; (2) partial recovery, or one third of expected recovery time; (3) semi recovery, or two thirds of recovery time; and (4) full recovery. They found that at all phases, being a team athlete predicted faster recovery time. At phase two, active coping was also a predictor of faster recovery time. Two unexpected factors emerged at phase two to predict slower recovery time - having more social support and completing the rehabilitation program. The authors suggested that perhaps social support was important in the recovery process but did not speed up recovery time. Also, completing the rehabilitation program may have been an indication that athletes were doing too much too soon in their eagerness to return to play, thereby prolonging their recovery. At phase three, the 
significant predictors were vigor and use of more denial/emotion-focused coping. At phase four, previously suffering a serious nonsporting illness or injury, vigor, greater intensity of effort, and more confidence emerged as significant predictors of faster recovery. The authors pointed out that causal relationships could not be established in the study. Another limitation is that preinjury variables were not examined and taken into consideration in the prediction of recovery time. The authors noted the need to evaluate the efficacy of psychological interventions in reducing recovery time.

Ievleva and Orlick (1991) investigated psychological factors that may enhance recovery time from a sport injury. Athletes $(N=32)$ were ranked according to their recovery time from an injury and were divided into three groups: (1) fast healers $(n=6)$; (2) average healers $(n=16)$; and (3) slow healers $(n=10)$. The researchers found that fast healers retrospectively reported more goal setting, positive self-talk, and healing imagery than slow healers. Self-reported use of goal setting emerged as the most important variable among fast healers. Qualitative analyses indicated that daily goal setting was more related to recovery time than either the long-term or return-to-sport goals. A major limitation of this study is that participants were asked to retrospectively recall strategies they used during rehabilitation once they were already recovered. Also, athletes had different types of injuries, although the authors reported that recovery time was comparable. Finally, it is unclear how the researchers determined the parameters for "fast," “average,” and “slow” recovery for the injuries studied.

Suggestions for Implementing Psychological Interventions for Sport Injury Rehabilitation Since the response to injury is multifaceted and includes biological, psychological, and social components (Brewer et al., 2002; Mainwaring, 1999; Wiese-Bjornstal et al., 1998), numerous recommendations have been made for implementing psychological interventions with 
injured athletes to improve various aspects of recovery. Petitpas and Danish (1995) provided a framework for implementing such interventions. This framework consists of several phases, including a rapport-building phase, education phase (teaching of skills), skill-development phase, and practice and evaluation phase. They emphasized that practitioners should progress through the four phases while concurrently working with the athlete's support network. They also provided a list of several skills, or psychological techniques that other authors had found useful for injury rehabilitation including: attention control training, biofeedback, cognitive strategies, communication-skills training, crisis intervention, goal setting, grief counseling, imagery, progressive relaxation, psychological skills training, psychotherapy, stress inoculation training, systematic desensitization, and support system interventions.

Crossman (1997) stated that to make rehabilitation more productive, an athlete should make use of psychological strategies during down time, such as when taking whirlpool baths, getting taped, or getting massaged. To make rehabilitation "work” (p. 336), Crossman advocated a host of psychological strategies including: meaningful communication between the athlete, medical professional, and coach; relaxation; social support; monitoring progress; rewards for progress; finding a new role within the team structure; and stress management (incorporating cognitive restructuring, thought control, imagery, and simulations). O’Conner, Heil, Harmer, and Zimmerman (2005) argued that success in rehabilitation comes from a combination of: education about the injury and the rehabilitation process, goal setting, social support, and mental training (including relaxation, imagery, concentration, and realistically positive self-talk). They also said that a "remarkable recovery, which involves a recovery and return to sport that are substantially faster and more complete than the norm” (p. 206), requires that the athlete have heightened body awareness, enhanced pain management, excellent mental skills, and a new perspective on sport. 
Since an athlete's psychological concerns in regards to injury change over the course of rehabilitation, Striegel, Hedgpeth, and Sowa (1996) recommended that practitioners employ different strategies with athletes based on the length of rehabilitation that the injury requires. For a short-term rehabilitation ( 0 to 2 weeks), an athlete's major concern is stress. Thus stress management and systematic rationalization are techniques that should be used during this time. Systematic rationalization consists of athletes identifying stressors related to injury or rehabilitation, classifying the stressors as controllable/uncontrollable or important/unimportant, and processing these stressors (i.e., discuss impact and reframe if necessary). For a long-term rehabilitation (longer than 2 weeks), an athlete should use stress management and systematic rationalization to address stress, goal setting to address motivation, and social support strategies to enhance compliance. If an athlete has a career ending injury, he/she should use the same strategies as a long-term injured athlete in addition to career counseling for career termination.

Taylor and Taylor (1997) went further than other researchers in their recommendations for psychological interventions during injury rehabilitation by providing an example of a comprehensive mental skills training intervention for injured athletes, called a Psychological Rehabilitation Program (PReP). The PReP begins with assessment of an athlete's psychological needs at their current stage of rehabilitation. The injured athlete should then identify two to three needs that they want to work on in physical therapy sessions, sport training, and at home. Each mental skills program should be individualized to the athlete's needs and preferences. The authors encourage athletes to select techniques based on “comfort and liking” (p. 299), however they suggested that rehabilitation imagery be part of every PReP because of its generalized and widespread benefits for recovery. The authors also suggested that athletes plan their use of psychological techniques by making a daily and weekly schedule. Some techniques that the 
authors discussed as possibilities to include in the PReP were: goal setting, relaxation, imagery, self-talk, social support, and confidence building.

Although the above suggestions for how to implement psychological skills training with injured athletes are based somewhat on the literature, many of the suggestions also appear to be based on practical experiences of the authors. While these sources of non-empirical information can be useful, experimental studies are needed to evaluate the efficacy of these approaches.

\section{Empirical Research on Psychological Interventions for Injury Rehabilitation}

Although textbook authors and researchers have strongly advocated psychological skills training during rehabilitation for injured athletes (as noted above), few methodologically sound, controlled studies of interventions with injured athletes have been conducted (Cupal, 1998).

Studies exploring psychological interventions with injured athletes will be presented below in the following sections: (1) perceptions of psychological interventions for injured athletes; (2) athlete self-reported use of coping/mental skills during injury recovery; and (3) intervention studies of mental skills with injured participants.

\section{Perceptions of Psychological Interventions for Injured Athletes}

In order to understand if psychological interventions would be acceptable to injured athletes as part of treatment, several studies were conducted to explore perceptions of such interventions. These studies presented a vignette about an injured athlete and described possible intervention strategies for that athlete. Myers, Peyton, and Jensen (2004) evaluated the treatment acceptability of behavioral (i.e., positive self-talk, imagery, relaxation, goal setting) and counseling (i.e., empathy, support, emotional awareness) interventions with injured collegiate football players $(N=40)$. Both interventions were rated as moderately acceptable, and there was no significant difference in acceptability between the two interventions. 
In two experiments which explored perceptions of college students $(N=161)$ and injured athletes $(N=20)$ regarding three psychological interventions for injured athletes (i.e., goal setting, imagery, and counseling), positive perceptions for all three interventions were obtained (Brewer, Jeffers, Petitpas, \& Van Raalte, 1994). Females displayed a significantly more positive perception of all three interventions than males. Overall, participants displayed a slight preference for goal setting. The researchers suggested that the reason for this preference may be because setting goals is a more natural part of the athlete's daily routine and that goal setting does not typically involve a large time commitment. Also, injured athletes can view themselves as active participants in goal setting more than imagery or counseling.

In another study, Francis, Andersen, and Maley (2000) explored views of both physiotherapists $(n=57)$ and male professional athletes $(n=28)$ in Australia on characteristics of athletes who cope well with injury as well as the usefulness of incorporating psychological skills into rehabilitation. While both physiotherapists and athletes viewed goal setting and encouraging positive thoughts as important, they perceived that the least effective selected techniques for rehabilitation were relaxation and visualization. They also both thought that they did not need knowledge about these techniques for rehabilitation. The authors further pointed out that this may actually be an indication of the lack of understanding of how these techniques could be beneficial to recovery (i.e., pain management, stress management) as well as how the techniques could be incorporated into rehabilitation, considering the physiotherapists rated “understanding stress/anxiety” as important to recovery. The findings of this study were generally in agreement with those of an earlier study using the same basic methodology with athletic trainers $(N=115)$ in the United States (Wiese, Weiss, \& Yukelson, 1991). The 
limitations of this study were that the sample size was relatively small and only male athletes were used.

Ninedek and Kolt (2000) conducted a similar study to that of Francis, Andersen, and Maley (2000). However, these researchers chose to examine perceptions of psychological interventions for injured athletes among physiotherapists $(N=150)$ in Australia who had a specific background in sports physiotherapy. These authors pointed out that in the Francis et al. study, only 4 of the 57 participants had formal postgraduate training in sports physiotherapy. In accordance with the findings of Francis et al., Ninedek and Kolt also found that physiotherapists rated the psychological skills of goal setting and encouraging positive thoughts as important and were more neutral about the skills of relaxation and imagery. In both studies (Francis et al.; Ninedek \& Kolt), positive attitude, intrinsic motivation, and determination/mental toughness were rated as important characteristics of athletes who coped well with injury, with willingness to listen to the physiotherapist emerging as the most important factor.

Athlete Self-reported Use of Coping/Mental Skills During Injury Recovery

Researchers have explored various coping/mental skills that injured athletes reportedly use during injury recovery as well as how these skills might affect the athletes' recovery. Scherzer et al. (2001) examined athletes’ $(N=54)$ self-reported use of goal setting, positive selftalk, and healing imagery in injury rehabilitation in relation to adherence. The researchers found that goal setting was positively related to home exercise completion and trainer adherence ratings. Also, positive self-talk was positively associated with home exercise completion. Selfreported use of healing imagery was not significantly related to any of the adherence measures. The authors suggested that imagery may contribute positively to recovery, but not affect adherence. This study may have benefited from asking about types of imagery other than healing 
(such as imagining rehabilitation exercises or sport performances). A limitation of the measure used in this study was that self-talk and imagery use were measured with a single item. Also, results were correlational and causality could not be inferred.

Other studies have looked at self-reported use of imagery and the impact on injury outcome variables. Sordoni, Hall, and Forwell (2002) examined how different types of imagery (i.e., motivational, cognitive, and healing) related to injured athletes' $(N=217)$ self-efficacy in rehabilitation. Motivational imagery involves imagining achieving a specific goal and the emotions and arousal associated with performing. Cognitive imagery involves imagining specific skills. Healing imagery involves imagining positive physiological changes and healing processes in the body. Healing imagery was the only type of imagery that was significantly related to selfefficacy for rehabilitation; however, the amount of variance accounted for in self-efficacy by healing imagery was only about $4 \%$. The authors found that athletes scored high on self-efficacy for rehabilitation, so there may have been a ceiling effect in regards to self-efficacy.

In a follow-up study, Milne, Hall, and Forwell (2005) also examined the relationship between imagery use and self-efficacy, but divided self-efficacy for rehabilitation into two factors: (1) task self-efficacy and (2) coping self-efficacy. Task self-efficacy refers to the belief in one's ability to perform rehabilitation exercises. Coping self-efficacy includes the belief in one’s ability to overcome barriers and schedule/plan for rehabilitation sessions. Another difference in this study was that the researchers used regression analyses to examine the predictive relationships between imagery and self-efficacy as opposed to correlational analyses in the previous study (Sordoni et al., 2002). The researchers found that for a sample of injured athletes $(N=237)$, cognitive imagery (mental rehearsal of rehabilitation exercises) was a significant predictor of task self-efficacy (belief in one's ability to perform rehabilitation 
exercises). However, the percentage of variance accounted for in self-efficacy by imagery was small (1.8\%). No other significant predictors emerged between the types of imagery and task or coping self-efficacy. The authors concluded that "other sources of self-efficacy appear to be more important than the use of imagery” (p. 160).

In a qualitative study of imagery use by injured athletes, Driediger, Hall, and Callow (2006) examined the "where, when, why, and what” of injured athletes' $(N=10)$ imagery in rehabilitation. The authors found that the athletes who used imagery believed that it served many valuable functions for injury recovery, which contrasts with other findings in the literature (e.g., Francis et al., 2000). Athletes reported motivational imagery (i.e., imagining themselves achieving a goal such as being fully recovered) as the most important and most commonly used form of imagery during rehabilitation. Athletes reported using imagery for a variety of purposes including: to help maintain their ability to perform sport skills, motivation, focus, healing, pain management, and injury prevention.

Intervention Studies of Mental Skills with Injured Participants

Empirical studies exploring the actual use or learning of psychological skills by injured athletes have been conducted with a variety of psychological interventions such as goal setting, relaxation, imagery, cognitive restructuring, and stress management. Some researchers examined a single intervention with injured participants while others explored a multimodal intervention (employing several interventions together as a "package”). Studies of the various types of psychological interventions with injured participants are presented below in the following four sections: (1) goal setting; (2) relaxation; (3) imagery; and (4) multimodal interventions. Many of the studies below were conducted with injured athletes, while some were conducted with a nonathlete population. 
Goal setting. A goal is defined as the "object or aim of an action” (Locke, Shaw, Saari, \& Latham, 1981, p. 126). In reviewing goal setting research in sport and physical activity settings, Burton, Naylor, and Holliday (2001) found a 78.6\% effectiveness rate for performance enhancement. After reviewing the literature, Gilbourne and Taylor (1998) proposed five essential principles for a task-oriented goal setting program for injured athletes. The principles included: (1) encouraging athletes to use goal setting as a transferable skill across situations; (2) helping athletes establish rehabilitation schedules; (3) providing opportunities for recording goals and evaluating them; (4) involving athletes in decision making when setting goals; and (5) ensuring that goals and progress are self-referenced. Goals should be specific, measurable, and realistic. Athletes should set both long- and short-term goals, set all three types of goals (i.e., outcome, performance, and process), and provide strategies and target dates for goals (Weinberg \& Gould, 2007).

Theodorakis, Malliou, Papaioannou, Beneca, and Filactakidou (1996) examined the effects of setting personal goals, perceived self-efficacy, ability (i.e., injured or non-injured), and self-satisfaction (i.e., how satisfied the person would be if they achieved a certain level of performance) on the performances of individuals ( $N=91$ females) on a knee extension task. Participants were divided into three groups: (1) an injured experimental group ( $n=32)$; (2) a non-injured experimental group ( $n=29)$; and (3) a control group of non-injured women $(n=30)$. Knee extension was measured over four trials using a Cybex 6000 isokinetic dynamometer as the outcome measure. The experimental groups were asked to set goals in between each trial and the control group did not set goals. There were significant performance improvements for both of the groups that set the personal goals, with no significant differences between the two experimental groups. The control group showed reduced performance from trial to trial. Correlational analyses 
showed that those participants with high self-efficacy and high self-satisfaction set higher personal goals and also performed better. The authors proposed that personal goal setting combined with strategies that increase self-efficacy may help athletes decrease recovery time from injury, help athletes overcome fears, increase their effort, and build their confidence.

In a similar study, Theodorakis, Beneca, Malliou, and Goudas (1997) examined the effects of a goal-setting program on the injury rehabilitation process. Participants $(N=37)$ were student-athletes who had received arthroscopic knee surgery 6-8 weeks prior to the study and were again tested on knee extension. Those in the experimental group $(n=20)$ were given feedback about their performance from the previous session and then set specific personal goals at the beginning of each rehabilitation session, while those in the control group did not receive feedback regarding their performance and did not set goals. The difference between this study and their previous study was that it covered the whole rehabilitation process, a four week period, rather than only part of the rehabilitation program. Once again, results indicated that athletes performed better when they incorporated goal-setting in their rehabilitation programs. The authors also examined changes in psychological variables amongst the two groups. Self-efficacy increased for both groups across trials, while anxiety decreased across trials for both groups. There was an interaction effect of group by trial for satisfaction with performance. Satisfaction with performance increased over the four trials for the experimental group, but not the control group.

Evans and Hardy (2002a) examined the effects of a five-week goal setting intervention on athletes' rehabilitation adherence, self-efficacy, belief in treatment efficacy, and psychological response variables labeled dispirited (i.e., feelings of apathy and loss of motivation) and reorganization (i.e., increased confidence and a sense of psychological 
recovery). Participants $(N=39)$ were matched on six variables (i.e., attending physiotherapist, nature of the injury, rehabilitation stage, sport, level of participation, and gender) and randomly assigned to one of three groups: goal setting intervention, social support control, and control. Based on athlete self-report of adherence, the goal setting group adhered significantly more to the rehabilitation program than the other two groups. The goal setting group also had the highest ratings of self-efficacy among the three conditions. There was a significant increase across time for reorganization and a decrease across time for feeling dispirited among all three groups. Therefore, affect and confidence improved over time, but there were no group differences. Evans and Hardy (2002b) also conducted a qualitative follow-up to this goal setting intervention study to enhance the interpretability and meaningfulness of the findings. They interviewed participants $(N=9)$, focusing on rehabilitation adherence, psychological and emotional responses, and social support. Participants were also asked to reflect on their overall injury experience. This follow-up study highlighted the importance of individual difference variables in response to injury and to the intervention, such as inability to train, weight gain, slowness of progress, and goal type preference (i.e., long- or short-term and outcome or performance goals). It also highlighted the importance of the interaction of person and situation variables, including support from coaches and club, and physiotherapist support. Therefore, both the individual and his/her situation should be taken into account when designing an intervention. Relaxation. Relaxation training is a tool to help injured athletes reduce anxiety and muscle tension. It can help injured athletes feel more in control of their tension and pain levels (Taylor \& Taylor, 1997). Relaxation techniques that may be useful for injured athletes include: diaphragmatic breathing, progressive muscle relaxation, passive relaxation, and autogenic training (Crossman, 2001; Taylor \& Taylor). 
In a study in Thailand (Roykulcharoen \& Good, 2004), participants ( $N=102)$ undergoing abdominal surgery were randomly assigned to a relaxation experimental group or a control group. State and trait anxiety were measured pre-surgery (one day before surgery). The sensation and distress of pain were measured post-surgery, once prior to the first ambulation (walking around) after surgery and again after the ambulation and intervention. Prior to surgery, the experimental group listened to a tape of systematic relaxation, where they were asked to relax each major body part in succession, starting from the feet and moving up the body to the head. After the first ambulation, those in the experimental group listened to the tape for 15 minutes, while those in the control group were told to rest quietly in bed for 15 minutes. Results indicated that the relaxation group reported significantly less post-test sensation and distress of pain than the control group. Relaxation did not result in significantly less anxiety for the relaxation group compared to the control group, but differences in anxiety were in the expected direction (i.e., the mean for state anxiety was lower for the relaxation group than the control group). In contrast to the above study, another study with female office workers with chronic neck pain $(N=393)$ did not find a difference in ratings of pain intensity among three groups (Viljanen et al., 2003). The three groups consisted of participants who received dynamic muscle training $(n=135)$, those who received relaxation training $(n=128)$, and those who did ordinary activity $(n=130)$.

Hough and Kleinginna (2002) conducted an individualized relaxation intervention with patients with spinal cord injuries $(N=6)$. An individualized treatment plan was crafted for participants based on information from their medical record, clinical psychological interview, and consultation with interdisciplinary team members (from a variety of fields including medicine, physical therapy, and psychology, among others). The treatment plan also took into account level of spinal cord injury, physical ability, and preferences for interventions. 
Satisfaction with the interventions and willingness to use them after treatment completion were assessed (via verbal self-report). The authors presented six cases, among which each patient had different goals for relaxation (e.g., to reduce anxiety, increase coping ability, reduce pain) and each learned different relaxation techniques based on their treatment plan and preferences (e.g., relaxation imagery, meditation, diaphragmatic breathing, progressive muscle relaxation). The analysis of case studies revealed that it was efficacious to take level of functioning and preferences into account when personalizing relaxation programs, as all patients improved in some aspect (physical or psychological). Case study analyses such as this one provide insight into the effects of interventions. However, larger sample experimental studies are needed to better analyze treatment efficacy.

Imagery. Imagery is a form of simulation in which experiences are created or recreated in the mind. A positive relationship between imagery and performance has been found in case studies, qualitative, and quantitative research (see Weinberg \& Gould, 2007). For injured athletes, imagery can be a valuable tool to enhance rehabilitation and return to sport. Several types of imagery may be useful to injured athletes (Green, 1999; Taylor \& Taylor, 1997) to include: (1) healing imagery, in which athletes imagine the body mending the injured area; (2) soothing imagery, in which athletes picture themselves in a relaxing and pleasant setting; (3) rehabilitation imagery, during which athletes imagine themselves performing rehabilitation exercises; and (4) performance imagery, in which athletes picture themselves practicing or competing in sport. This mental rehearsal of sport skills can especially be important to injured athletes since they cannot physically practice. Performance imagery can allow injured athletes to work on technique, tactics, and competitive performance over the course of rehabilitation (Taylor \& Taylor). 
Cupal and Brewer (2001) examined the effects of a guided imagery intervention with a relaxation component on knee strength, reinjury anxiety, and pain following anterior cruciate ligament reconstructive surgery. Participants $(N=30)$ were recreational and competitive athletes and were divided into three equal groups $(n=10)$ : (1) the treatment group, who received 10 individual sessions of relaxation and guided imagery over a 6-month period; (2) the placebo group, who received attention, encouragement, and support from a clinician at the sports medicine facility in addition to being asked to visualize a peaceful scene for 10-15 minutes per day; and (3) the control group, who received standard care (physical therapy). The imagery scripts were all different and based on the phase of rehabilitation that the patient was in. However, all scripts consisted of the following components: (a) the physiological processes at work, (b) suggestions promoting positive emotional coping responses, and (c) inclusion of all senses to enhance the vividness of the imagery. At approximately 6 months post-surgery, participants in the intervention group had significantly greater knee strength, less reinjury anxiety, and less pain than participants in the control and placebo groups. There were no differences between the control and placebo groups. The researchers concluded that an intervention similar to the one used in this study may accelerate physical recovery as well as psychological recovery.

Handegard, Joyner, Burke, and Reimann (2006) used both quantitative and qualitative methodology to explore the effects of imagery on self-confidence and fear of returning to sport with two injured male athletes. Participants completed three imagery training sessions and were given an audio tape to listen to with the imagery script on it. The script was a combination of relaxation and healing imagery. According to imagery logs that the participants kept, they did imagery twice daily for a total of 25 minutes per day. Qualitative statements indicated that the 
participants felt that the imagery helped give them a sense of control in their recovery and feel more mentally prepared to return to sport. They attributed $30-40 \%$ of their recovery to the imagery intervention. These percentages should be interpreted with caution, as they were estimated by the participants in an exit survey question and not determined by statistical measures. Both participants expressed concern, but did not have a fear of returning to play. This may have been due to the moderate severity of the injuries sustained by the athletes, with a recovery time of only two weeks. Although stated as purposes of the study, the researchers did not specifically discuss the relationship between the imagery intervention and trait and state sport-confidence. Also, there was no baseline measure of confidence prior to learning the imagery; therefore, any increase in confidence should not be attributed to the intervention.

Another study found that mental imagery helped to prevent loss of strength due to immobilization of the forearm (Newsom, Knight, \& Balnave, 2003). Participants’ nondominant forearms were immobilized for 10 days. Those in the experimental group $(n=9)$ were asked to perform mental imagery of squeezing a rubber ball for five minutes three times per day. Those in the control group $(n=8)$ were not asked to do imagery. There were no significant differences between groups in loss of grip strength post-intervention; however, the loss of grip strength in the control group (16.3\%) was greater than that of the intervention group (1.5\%). Also, there was no significant decrease in the wrist-flexion or -extension strength for the intervention group, but there was a significant decrease for those in the control group. The small sample size was a limitation in this study.

Alden, Dale, and DeGood (2001) manipulated the affect quality (i.e., positive vs. negative) and directional focus (i.e., internal vs. external) of imagery to examine the effects on college students' $(N=50)$ perceived pain during a cold-pressor test. The directional focus of the 
imagery refers to whether the content of the imagery is specific to the internal painful body part or imagined as completely external to the body. According to ratings on a visual analogue scale (VAS), participants experienced the least amount of pain in the condition where they listened to a tape of imagery with positive affect and an external focus. Participants in this group also had a longer pain tolerance. The authors concluded that positive distracting imagery can be useful in the control of pain and appears to be more effective than negative imagery and imagery that is focused on internal bodily processes. Since this study was performed in a lab, results may not generalize to individuals with actual injury pain.

Multimodal interventions. Weinberg and Gould (2007) stated, “the most important psychological skills to learn for rehabilitation are goal setting, positive self-talk, imagery visualization, and relaxation training” (p. 457). A multimodal approach to sport injury rehabilitation would include using a combination of psychological skills interventions to enhance recovery from injury. Multimodal interventions for the rehabilitation of injured athletes have been supported in the literature (e.g., Cupal, 1998; Evans, Hardy, \& Flemming, 2000).

Ross and Berger (1996) investigated the efficacy of a cognitive-behavioral intervention, specifically stress inoculation training (SIT), on injured athletes’ postsurgical anxiety, pain, and physical functioning. Male athletes $(N=60)$, who received arthroscopic knee surgery for a meniscus tear, were alternately assigned to either a treatment group (physical therapy and SIT) or a control group (physical therapy only). The participants in the treatment group attended two 1hour SIT sessions. The SIT procedures involved three components: (1) conceptualization, or the rationale for understanding their cognitive and emotional responses to injury; (2) skill acquisition, where participants learned skills such as self-monitoring of cognitive and emotional responses, relaxation techniques, imagery, and positive self-talk; and (3) application, when 
participants were instructed on when, where, and how to practice these strategies daily. The researchers found that both state anxiety and pain decreased over time but more rapidly for participants in the treatment condition than the control condition. They also discovered that the treatment group evidenced significantly less anxiety and reported significantly less pain than the control group. Finally, the researchers found that the mean number of days to recovery for the treatment group was significantly less than the mean number of recovery days for the control group. The researchers concluded that a cognitive-behavioral intervention could enhance the psychological and physical rehabilitation of injured athletes.

Johnson (2000) explored the effectiveness of a short-term psychological intervention for a sample of competitive athletes with long-term injuries. The participants in the experimental group ( $n=14$ ) attended three different 15- to 25-minute training sessions. One session addressed stress management and cognitive control, another focused on goal setting skills, and another on relaxation/guided imagery. The control group $(n=44)$ did not receive any form of psychological intervention. The researchers used mood level as the main outcome variable. At the midpoint and at the end of rehabilitation, the experimental group had a higher overall mood state than did the control group. From the results of the study, Johnson concluded that a short-term psychological intervention including a combination of stress management, goal setting, and relaxation/guided imagery, seemed to have a significantly elevating effect on the injured patients’ overall mood during and at the end of the rehabilitation period. However, when participants were asked to rate their readiness to return to sport based on three statements (one resembling each intervention’s purpose), the relaxation/guided imagery statement was the only one that showed statistically significant differences between the control and experimental groups. This study has limited generalizability because the experimental group consisted of all men. Also, the high overall 
mood level of the experimental group might have been an effect of the attention they received during the intervention. The difference in group size was another weakness of the study.

Evans, Hardy, and Fleming (2000) qualitatively explored the efficacy of a multi-modal intervention involving consultations with three injured athletes, using techniques such as goal setting, simulation training, visualization, social support, and verbal persuasion. The authors explored strategies that participants found useful throughout different phases of rehabilitation (i.e., early, mid, and late phases). Emotional support was perceived as important to individuals during times of setbacks, when progress was slow, and when other stressors were being experienced. Goal setting was used by the athletes to enhance motivation and adherence during the early and mid phases of rehabilitation. Toward the latter phase and during reentry to competitive sport, goal setting was used to enhance self-efficacy. Support was provided for the use of both long- and short-term goals, as well as process and performance goals. In particular, process goals helped focus attention on specific features of a given task, and performance goals provided structure and specific targets for participants' rehabilitation. The study also highlighted the importance of goal flexibility. Athletes felt that confidence in the injured body part during the reentry phase was critical, and simulation training, imagery (particularly of situations in which the injury occurred), and verbal persuasion helped build confidence with the athletes.

Rock and Jones (2002) explored the impact of six counseling sessions over 12 weeks post-surgery for three injured athletes using a single subject design. The researchers used the following outcome measures: mood, perceived rehabilitation progress, pain ratings, and social support. Semistructured interviews were conducted 12 weeks post-surgery. Both quantitative and qualitative analyses were used. Two of the three participants had favorable impressions of the intervention. By the end of the rehabilitation period monitored, mood disturbance had fallen 
below presurgery levels for all participants. Also, perceptions of rehabilitation progress increased while pain ratings decreased, although both fluctuated, particularly in response to setbacks. A caution with these findings is that the above variables (mood, progress, and pain) are likely to improve over time regardless of receiving an intervention, and it is difficult to determine whether the changes were due to the intervention or to time. With regards to support, the female participant recorded higher levels of listening and emotional support. There was also qualitative evidence that the intervention was a source of informational support. The researchers also found that the counseling-skills intervention reduced the negative impact of setbacks on mood and perceptions of rehabilitation. The researchers came across an unexpected finding that some participants saw surgery and rehabilitation as a positive experience (i.e., self-growth, learning experience). They hypothesized that the intervention might have provided participants with the support in reframing their experiences and an opportunity to test out and validate their positive interpretations. The researchers also highlighted the importance of considering individual differences during counseling, as each participant’s experience was unique.

In another single subject study employing a multiple baseline ABCA design, Naoi (2003) explored the effects of cognitive and relaxation interventions on injured athletes' $(N=7)$ mood, optimism, adherence, compliance, recovery rate, and pain. Naoi concluded that there was a probability that three participants benefited from the cognitive intervention, three benefited from the relaxation intervention, and two benefited from both interventions. There were several significant correlations among the dependent variables in the study, which included: a negative relationship between pain and depression at pre-test (i.e., those with high pain were less depressed), a positive relationship between optimism at pre-test and recovery rate assessed by athletic trainers at the post-test (i.e., high optimism related to a faster recovery), a negative 
relationship between tension and recovery rate (i.e., higher tension related to slower recovery), and a negative relationship between pain and compliance to rehabilitation (i.e., those with lower pain were more compliant).

Naoi (2003) also found that there were large individual differences in response to the interventions, and suggested that future researchers employ single subject studies to further explore individual differences such as taking into account preferences and needs for specific interventions. A manuscript has recently been published on part of Naoi’s data (Naoi \& Ostrow, 2008). In this manuscript, Naoi and Ostrow reported that participants found the cognitive intervention to be more effective on physiological and psychological recovery than the relaxation intervention (participants rated this effectiveness on a scale of 1 to 10). However, these results were greatly affected by one of the participants rating the relaxation effectiveness as very low. Naoi and Ostrow's study could have benefited from qualitative interviews to find out more information about how the interventions benefited (or did not benefit) the athletes physically and psychologically.

An advantage to studying multimodal interventions is that techniques can be combined so that participants can potentially receive more benefits than if learning only one skill. For research purposes, however, multimodal interventions can make it difficult to distinguish which skills are most/least beneficial and which aspects of the intervention are actually exerting effects.

\section{Summary}

An injured athlete's psychological response to injury is typically multifaceted (Brewer, et al., 2002; Mainwaring, 1999; Wiese-Bjornstal et al., 1998); therefore, an intervention to address the various components of psychological response to injury (cognitive, emotional, and behavioral) is necessary. Self-reported use of goal setting, imagery, and positive self-talk has 
been associated with positive rehabilitation outcomes (Ievleva \& Orlick, 1991; Scherzer et al., 2000). Therefore, interventions employing these techniques should affect outcomes such as adherence and recovery time. Many psychological interventions for injured athletes have been proposed in the literature (e.g., O’Conner et al., 2005; Taylor \& Taylor, 1997). However, there are few methodologically sound studies that examine the effects of actual psychological interventions with injured athletes (Cupal, 1998). Of the studies that have been conducted examining interventions such as goal setting (Evans \& Hardy, 2002a), relaxation (Roykulcharoen, 2004), imagery (Cupal \& Brewer, 2001), stress management (Ross \& Berger, 1996), and counseling (Rock \& Jones, 2002), most have found at least some positive effects of the intervention on psychological variables and rehabilitation outcomes.

Psychological factors, such as self-efficacy, have been found to influence rehabilitation outcomes, such as adherence and recovery time from injury. Self-efficacy of injured athletes has also been found to improve as a result of psychological interventions such as goal setting (Evans \& Hardy, 2002a). Therefore, self-efficacy appears to be an important variable to study in relation to psychological interventions and rehabilitation outcomes.

A major gap in the literature on psychological interventions with injured athletes is how to best individualize such interventions so as to provide maximal benefits to an athlete. Some authors advise that practitioners working with injured athletes choose psychological skills based on athletes' “needs” and “preferences” (e.g., Naoi, 2003; Taylor \& Taylor, 1997). However, there is little mention of how to make such decisions.

Should athletes learn to apply mental skills to rehabilitation that they are already proficient at using in sport, or should they learn new skills that they may never have been exposed to? Since athletes’ psychological responses to injury are so varied (Brewer \& Cornelius, 
2003; Shapiro, 2005; Wiese-Bjornstal et al., 1998) and athletes’ responses to psychological interventions are also varied (Naoi, 2003; Rock \& Jones, 2002), future studies should explore how to tailor interventions to injured athletes so that they are satisfied with the intervention and benefit from it. In addition, more qualitative studies are needed to provide insight into injured athletes’ experiences with mental skills interventions.

\section{References}

Albinson, C. A., \& Petrie, T. A. (2003). Cognitive appraisals, stress, and coping: Preinjury and postinjury factors influencing psychological adjustment to sport injury. Journal of Sport Rehabilitation, 12, 306-322.

Alden, A. L., Dale, J. A., \& DeGood, D. E. (2001). Interactive effects of the affect quality and directional focus of mental imagery on pain analgesia. Applied Psychophysiology and Biofeedback, 26, 117-126.

Bandura, A. (1977). Self-efficacy: Toward a unifying theory of behavioral change. Psychological Review, 84, 191-215.

Brewer, B. W. (1994). Review and critique of models of psychological adjustment to athletic injury. Journal of Applied Sport Psychology, 6, 87-100.

Brewer, B. W., Andersen, M. B., \& Van Raalte, J. L. (2002). Psychological aspects of sport injury rehabilitation: Toward a biopsychosocial approach. In D. L. Mostofsky and L. D. Zaichkowsky (Eds.), Medical and psychological aspects of sport and exercise (pp. 4154). Morgantown, WV: Fitness Information Technology.

Brewer, B. W., \& Cornelius, A. E. (2003). Psychological factors in sports injury rehabilitation. In W. R. Frontera (Ed.), Rehabilitation of sports injuries: Scientific basis (pp. 160-183). Malden, MA: Blackwell Science. 
Brewer, B. W., Cornelius, A. E., Van Raalte, J. L., Brickner, J. C., Sklar, J. H., Corsetti, J. R., Pohlman, M. H., Ditmar, T. D., \& Emery, K. (2004). Rehabilitation adherence and anterior cruciate ligament reconstruction outcome. Psychology, Health, \& Medicine, 9, 163-175.

Brewer, B. W., Cornelius, A. E., Van Raalte, J. L., Petitpas, A. J., Sklar, J. H., Pohlman, M. H., Krushell, R. J., \& Ditmar, T. D. (2003). Protection motivation theory and adherence to sport injury rehabilitation revisited. The Sport Psychologist, 17, 95-103.

Brewer, B. W., Jeffers, K. E., Petitpas, A. J., \& Van Raalte, J. L. (1994). Perceptions of psychological interventions in the context of sport injury rehabilitation. The Sport Psychologist, 8, 176-188.

Burton, D., Naylor, S., \& Holliday, B. (2001). Goal setting in sport: Investigating the goal effectiveness paradox. In R. N. Singer, H. A. Hausenblas, \& C. M. Janelle (Eds.), Handbook of sport psychology ( $2^{\text {nd }}$ ed., pp. 497-528). New York: John Wiley \& Sons, Inc.

Crossman, J. (1997). Psychological rehabilitation from sports injuries. Sports Medicine, 23, 333339.

Crossman, J. (2001). Coping with sport injuries: Psychological strategies for rehabilitation. New York: Oxford University Press Inc.

Cupal, D. D. (1998). Psychological interventions in sport injury prevention and rehabilitation. Journal of Applied Sport Psychology, 10, 103-123.

Cupal, D. D., \& Brewer, B. W. (2001). Effects of relaxation and guided imagery on knee strength, reinjury anxiety, and pain following anterior cruciate ligament reconstruction. Rehabilitation Psychology, 46, 28-43. 
Daly, J. M., Brewer, B. W., Van Raalte, J. L., Petitpas, A. J., \& Sklar, J. H. (1995). Cognitive appraisal, emotional adjustment, and adherence to rehabilitation following knee surgery. Journal of Sport Rehabilitation, 4, 22-30.

Driediger, M., Hall, C., \& Callow, N. (2006). Imagery use by injured athletes: A qualitative analysis. Journal of Sport Sciences, 24, 261-271.

Evans, L., \& Hardy, L. (2002a). Injury rehabilitation: A goal setting intervention study. Research Quarterly for Exercise and Sport, 73, 310-319.

Evans, L., \& Hardy, L. (2002b). Injury rehabilitation: A qualitative follow-up study. Research Quarterly for Exercise and Sport, 73, 320-329.

Evans, L., \& Hardy, L. (1995). Sport injury and grief responses: A review. Journal of Sport and Exercise Psychology, 17, 227-245.

Evans, L., Hardy, L., \& Fleming, S. (2000). Intervention strategies with injured athletes: An action research study. The Sport Psychologist, 14, 188-206.

Francis, S. R., Andersen, M. B., \& Maley, P. (2000). Physiotherapists’ and male professional athletes’ views on psychological skills for rehabilitation. Journal of Science and Medicine in Sport, 3, 17-29.

Gilbourne, D., \& Taylor, A. H. (1998). From theory to practice: The integration of goal perspective theory and life development approaches within an injury-specific goal-setting program. Journal of Applied Sport Psychology, 10, 124-139.

Green, L. B. (1999). The use of imagery in the rehabilitation of injured athletes. In D. Pargman (Ed.), Psychological bases of sport injuries (2nd ed., pp. 235-251). Morgantown, WV: Fitness Information Technology.

Handegard, L. A., Joyner, B. A., Burke, K. L., \& Reimann, B. (2006). Relaxation and guided 
imagery in the sport rehabilitation context. Journal of Excellence, 11, 146-164.

Hough, S., \& Kleinginna, C. (2002). Individualizing relaxation training in spinal cord injury: Importance of injury level and person factors. Rehabilitation Psychology, 47, 415-425.

Ievleva, L., \& Orlick, T. (1991). Mental links to enhanced healing: An exploratory study. The Sport Psychologist, 5, 25-40.

Johnson, U. (2000). Short-term psychological intervention: A study of long-term-injured competitive athletes. Journal of Sport Rehabilitation, 9, 207-218.

Kubler-Ross, E. (1969). On death and dying. New York: Macmillan.

Levy, A. R., Polman, R. C. J., \& Clough, P. J. (2008). Adherence to sport injury rehabilitation programs: An integrated psycho-social approach. Scandinavian Journal of Medicine and Science in Sports, 18, 798-809.

Levy, A. R., Polman, R. C. J., Clough, P. J., \& McNaughton, L. R. (2006). Adherence to sport injury rehabilitation programmes: A conceptual review. Research in Sports Medicine, 14, 149-162.

Lippke, S., Ziegelmann, J. P., \& Schwarzer, R. (2004). Behavioral intentions and action plans promote physical exercise: A longitudinal study with orthopedic rehabilitation patients. Journal of Sport and Exercise Psychology, 26, 470-483.

Locke, E. A., Shaw, K. N., Saari, L. M., \& Latham, G. P. (1981). Goal setting and task performance. Psychological Bulletin, 90, 125-152.

Maddux, J. E., \& Rodgers, R. W. (1983). Protection motivation and self-efficacy: A revised theory of fear appeals and attitude change. Journal of Experimental Social Psychology, 19, 469-479.

Mainwaring, L. M. (1999). Restoration of self: A model for the psychological response of 
athletes to severe knee injuries. Canadian Journal of Rehabilitation, 12, 145-156.

Milne, M., Hall, C., \& Forwell, L. (2005). Self-efficacy, imagery use, and adherence to rehabilitation by injured athletes. Journal of Sport Rehabilitation, 14, 150-167.

Myers, C. A., Peyton, D. D., \& Jensen, B. J. (2004). Treatment acceptability in NCAA Division I football athletes: Rehabilitation intervention strategies. Journal of Sport Behavior, 27, 165-169.

Naoi, A. (2003). The effects of cognitive and relaxation interventions on injured athletes' mood, pain, optimism, and adherence to rehabilitation. Unpublished doctoral dissertation, West Virginia University, Morgantown, WV.

Naoi, A., \& Ostrow, A. (2008). The effects of cognitive and relaxation interventions on injured athletes’ mood and pain during rehabilitation. Athletic Insight, 10 (1). Retrieved May 4, 2008, from http://www.athleticinsight.com/Vol10Iss1/InterventionsInjury.htm.

Newsom, J., Knight, P., \& Balnave, R. (2003). Use of mental imagery to limit strength loss after immobilization. Journal of Sport Rehabilitation, 12, 249-258.

Ninedek, A., \& Kolt, G. S. (2000). Sport physiotherapists’ perceptions of psychological strategies in sport injury rehabilitation. Journal of Sport Rehabilitation, 9, 191-206.

Niven, A. (2007). Rehabilitation adherence in sport injury: Sport physiotherapists’ perceptions. Journal of Sport Rehabilitation, 16, 93-110.

O’Conner, E., Heil, J., Harmer, P., \& Zimmerman, I. (2005). Injury. In J. Taylor and G.S. Wilson (Eds.), Applying sport psychology (pp. 187-206). Champaign, IL: Human Kinetics.

Petitpas, A., \& Danish, S. J. (1995). Caring for injured athletes. In S. M. Murphy (Ed.), Sport psychology interventions (pp. 255-281). Champaign, IL: Human Kinetics.

Pizzari, T., Taylor, N. F., McBurney, H., \& Feller, J. A. (2005). Adherence to rehabilitation after 
anterior cruciate ligament reconstructive surgery: Implications for outcome. Journal of Sport Rehabilitation, 14, 201-214.

Quinn, A. M. \& Fallon, B. J. (2000). Predictors of recovery time. Journal of Sport Rehabilitation, 9, 62-76.

Rock, J. A., \& Jones, M. V. (2002). A preliminary investigation into the use of counseling skills in support of rehabilitation from sport injury. Journal of Sport Rehabilitation, 11, 284304.

Ross, M. J., \& Berger, R. S. (1996). Effects of stress inoculation training on athletes' postsurgical pain and rehabilitation after orthopedic injury. Journal of Consulting and Clinical Psychology, 64, 406-410.

Roykulcharoen, V., \& Good, M. (2004). Systematic relaxation to relieve postoperative pain. Journal of Advanced Nursing, 48, 140-148.

Scherzer, C. B., Brewer, B. W., Cornelius, A. E., Van Raalte, J. L., Petitpas, A. J., Sklar, J. H., Pohlman, M. H., Krushell, R. J., \& Ditmar, T. D. (2001). Psychological skills and adherence to rehabilitation after reconstruction of the anterior cruciate ligament. Journal of Sport Rehabilitation, 10, 165-172.

Schwarzer, R. (1992). Self-efficacy in the adoption and maintenance of health behaviours: Theoretical approaches and a new model. In R. Schwarzer (Ed.), Self-efficacy: Thought control of action (pp. 217-243). Washington, DC: Hemisphere.

Shapiro, J. L. (2005). Patterns of psychological adjustment to knee surgery. Unpublished master’s thesis, Springfield College, Springfield, MA.

Sordoni, C., Hall, C., \& Forwell, L. (2002). The use of imagery in athletic injury rehabilitation and its relationship to self-efficacy. Physiotherapy Canada, 54, 177-185. 
Striegel, D. A., Hedgpeth, E. G., \& Sowa, C. J. (1996). Differential psychological treatment of injured athletes based on length of rehabilitation. Journal of Sport Rehabilitation, 5, 330335.

Taylor, A. H., \& May, S. (1996). Threat and coping appraisal as determinants of compliance to sports injury rehabilitation: An application of protection motivation theory. Journal of Sports Sciences, 14, 471-482.

Taylor, J., \& Taylor, S. (1997). Psychological approaches to sports injury rehabilitation. Gaithersburg, MD: Aspen Publishers, Inc.

Theodorakis, Y., Beneca, A., Malliou, P., \& Goudas, M. (1997). Examining psychological factors during injury rehabilitation. Journal of Sport Rehabilitation, 6, 355-363.

Theodorakis, Y., Malliou, P., Papaioannou, A., Beneca, A., \& Filactakidou, A. (1996). The effect of personal goals, self-efficacy, and self-satisfaction on injury rehabilitation. Journal of Sport Rehabilitation, 5, 214-223.

Viljanen, M., Malmivaara, A., Uitti, J., Rinne, M., Palmroos, P., \& Laippala, P. Effectiveness of dynamic muscle training, relaxation training, or ordinary activity for chronic neck pain: Randomised controlled trial. British Medical Journal, 327, 475-479.

Weinberg, R. S., \& Gould, D. (2007). Foundations of sport and exercise psychology $\left(4^{\text {th }}\right.$ ed). Champaign, IL: Human Kinetics.

Wiese, D. M., Weiss, M. R., \& Yukelson, D. P. (1991). Sport psychology in the training room: A survey of athletic trainers. The Sport Psychologist, 5, 15-24.

Wiese-Bjornstal, D. M., Smith, A. M., Shaffer, S. M., \& Morrey, M. A. (1998). An integrated model of response to sport injury: Psychological and sociological dynamics. Journal of Applied Sport Psychology, 10, 46-69. 
APPENDIX B: Initial Interview

(To be stored separately from the data)

Date:

Participant Name:

Participant Code Number:

E-mail Address:

Phone number:

Interview Questions:

1. Basic demographic information

a. Gender

b. Age and year in school

c. Ethnicity

2. Briefly tell me about your athletic history.

a. Prompts - how many years playing, what sports, significant events

b. Current Sport and level

3. Tell me about your history of injuries.

a. Prompts - what injuries, age when injured, how long injuries kept you out of sport

4. Tell me about your current injury.

a. Prompts - injury type, how it happened, expected recovery time

b. Follow ups - What are some thoughts and feelings you have about your current injury? How has rehabilitation been so far?

5. What do you expect rehabilitation will be like?

6. What experiences, if any, do you have with sport psychology training?

a. Prompts - sport psych consulting, sport psych college classes

b. Follow up - Have you had any formal individualized mental skills training? 
APPENDIX C: Test of Performance Strategies-2

Reprinted with permission from Lew Hardy via e-mail (12/18/06)

$\mathbf{T}$

$\mathbf{0}$

\section{Test Of Performance Strategies ${ }^{\circledR}$}

$\mathbf{P}$

$\mathbf{S}$

\section{Participant Code Number:}

This questionnaire measures performance strategies used by athletes in various sport situations. Because individual athletes are very different in their approach to their sport, we expect the responses to be different. We want to stress, therefore, that there are no right or wrong answers. All that is required is for you to be open and honest in your responses.

Throughout the questionnaire, several terms are used which may have different meanings for different individuals. Because of this, these terms are defined below with specific examples to sport where appropriate. Please keep these definitions in mind when responding to items with these terms.

COMPETITION: a tournament/meet where individuals or teams perform against each other.

SKILL: a specific element of your sport performance. For example, free throw shooting in basketball or a jump in figure skating.

PERFORMANCE: your execution of specific sport skills during training and competition.

ROUTINE: a set of behaviours that is performed regularly in preparation for your performance in sport. An example may be going through specific stretches while listening to a song on your walkman prior to every performance.

WORKOUT: a structured practice session to work on various elements of your sport.

VISUALIZATION/IMAGERY/REHEARSAL: these terms refer to the act of picturing in your mind some aspect of your performance. An example would be seeing and feeling yourself execute a specific skill perfectly.

(c) Developed by P.R. Thomas, S.M. Murphy, and L. Hardy (v2x, 1999). 
Each of the following items describes a specific situation that you may encounter in your training and competition. Please rate how frequently these situations apply to you on the following scale:

$$
\begin{aligned}
& 1=\text { Never } \\
& 2=\text { Rarely } \\
& 3=\text { Sometimes } \\
& 4=\text { Often } \\
& 5=\text { Always }
\end{aligned}
$$

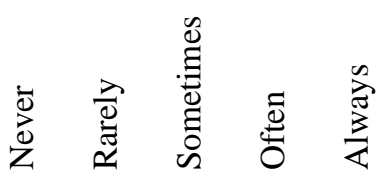

Please put a circle around your answer

1. I set realistic but challenging goals for practice.

2. I say things to myself to help my practice performance.

3. During practice I visualize successful past performances.

4. My attention wanders while I am training.

5. I practice using relaxation techniques at workouts.

6. During competition I set specific result goals for myself.

7. My self-talk during competition is negative.

8. I rehearse my performance in my mind before practice.

9. During competition I have thoughts of failure.

10. I use practice time to work on my relaxation technique.

11. I manage my self-talk effectively during practice.

12. I visualize my competition going exactly the way I want it to go.

13. I am able to control distracting thoughts when I am training.

14. I get frustrated and emotionally upset when practice does not go well.

15. I have specific cue words or phrases that I say to myself to help my performance during competition.

16. I evaluate whether I achieve my competition goals.

17. I set very specific goals for competition.

18. At practice, I can allow the whole skill or movement to happen naturally without concentrating on each part.

19. I keep my thoughts positive during competitions.

\begin{tabular}{|c|c|c|c|}
\hline 1 & 2 & & \\
\hline 1 & 2 & 3 & 4 \\
\hline 1 & 2 & 3 & 4 \\
\hline 1 & 2 & 3 & 4 \\
\hline 1 & 2 & 3 & 4 \\
\hline 1 & 2 & 3 & 4 \\
\hline 1 & 2 & 3 & 4 \\
\hline 1 & 2 & 3 & 4 \\
\hline 1 & 2 & 3 & 4 \\
\hline 1 & 2 & 3 & 4 \\
\hline 1 & 2 & 3 & 4 \\
\hline 1 & 2 & 3 & 4 \\
\hline 1 & 2 & 3 & 4 \\
\hline 1 & 2 & 3 & 4 \\
\hline 1 & 2 & 3 & 4 \\
\hline 1 & 2 & 3 & 4 \\
\hline 1 & 2 & 3 & 4 \\
\hline 1 & 2 & 3 & 4 \\
\hline 1 & 2 & 3 & 4 \\
\hline 1 & 2 & 3 & 4 \\
\hline 1 & 2 & 3 & 4 \\
\hline 1 & 2 & 3 & 4 \\
\hline
\end{tabular}

20. I say things to myself to help my competitive performance.

21. At competitions, I rehearse the feel of my performance in my imagination.

22. I manage my self-talk effectively during competition. 
23. I set goals to help me use practice time effectively.

24. At practice, when I visualize my performance, I imagine what it will feel like.

25. During practice I focus my attention effectively.

26. I set personal performance goals for a competition.

27. I motivate myself to train through positive self-talk.

28. I have trouble maintaining my concentration during long practices.

29. I talk positively to myself to get the most out of practice.

30. I have very specific goals for practice.

31. I imagine my competitive routine before I do it at a competition.

32. I imagine screwing up during a competition.

33. I talk positively to myself to get the most out of competitions.

34. I don't set goals for practices; I just go out and do it.

35. I rehearse my performance in my mind at competitions.

36. I have trouble controlling my emotions when things are not going well at practice.

37. My emotions keep me from performing my best at competitions.

38. My emotions get out of control under the pressure of competition.

39. At practice, when I visualize my performance, I imagine watching myself as if on a video replay.

40. I can allow the whole skill or movement to happen naturally in competition without concentrating on each part.

41. I use relaxation techniques as a coping strategy at competitions.

42. I can psych myself to perform well in practice.

43. I am able to perform skills at practice without having to consciously think about them.

44. I can get myself ready to perform when I am at competitions.

45. I have difficulty with my emotions at competitions.

46. During training sessions I use relaxation techniques to improve my performance.

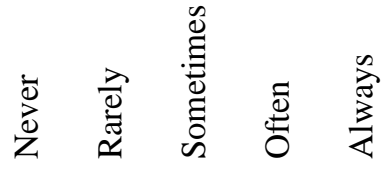

$\begin{array}{lllll}1 & 2 & 3 & 4 & 5 \\ 1 & 2 & 3 & 4 & 5\end{array}$

$\begin{array}{lllll}1 & 2 & 3 & 4 & 5\end{array}$

$\begin{array}{lllll}1 & 2 & 3 & 4 & 5\end{array}$

$\begin{array}{lllll}1 & 2 & 3 & 4 & 5\end{array}$

$\begin{array}{lllll}1 & 2 & 3 & 4 & 5\end{array}$

$\begin{array}{lllll}1 & 2 & 3 & 4 & 5\end{array}$

$\begin{array}{lllll}1 & 2 & 3 & 4 & 5\end{array}$

$\begin{array}{lllll}1 & 2 & 3 & 4 & 5\end{array}$

$\begin{array}{lllll}1 & 2 & 3 & 4 & 5\end{array}$

$\begin{array}{lllll}1 & 2 & 3 & 4 & 5\end{array}$

$\begin{array}{lllll}1 & 2 & 3 & 4 & 5\end{array}$

$\begin{array}{lllll}1 & 2 & 3 & 4 & 5\end{array}$

$\begin{array}{lllll}1 & 2 & 3 & 4 & 5\end{array}$

$\begin{array}{lllll}1 & 2 & 3 & 4 & 5\end{array}$

$\begin{array}{lllll}1 & 2 & 3 & 4 & 5\end{array}$

$\begin{array}{lllll}1 & 2 & 3 & 4 & 5\end{array}$

$\begin{array}{lllll}1 & 2 & 3 & 4 & 5\end{array}$

$\begin{array}{lllll}1 & 2 & 3 & 4 & 5\end{array}$

$\begin{array}{lllll}1 & 2 & 3 & 4 & 5\end{array}$

$\begin{array}{lllll}1 & 2 & 3 & 4 & 5\end{array}$

$\begin{array}{lllll}1 & 2 & 3 & 4 & 5\end{array}$

$\begin{array}{lllll}1 & 2 & 3 & 4 & 5\end{array}$

$\begin{array}{lllll}1 & 2 & 3 & 4 & 5\end{array}$


47. I need to monitor all the details of each move in order to successfully execute skills in practice.

48. I have difficulty controlling my emotions if I make a mistake at competitions.

49. Visual distractions during competition would affect my performance.

50. My emotions keep me from performing my best during practice.

51. My competition performance would be impaired by sleep loss.

52. I have difficulty getting into an ideal performance state during training.

53. I can psych myself to perform well in competitions.

54. I use relaxation techniques during competitions to improve my performance.

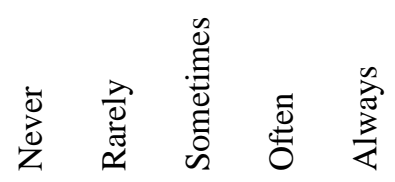

55. I can get myself “up” if I feel flat at practice.

56. I am unable to perform skills at competition without consciously thinking about them.

57. If I'm starting to "lose it” at a competition, I use a relaxation technique.

58. I can get my intensity levels just right for competition.

59. During practice, I can perform automatically without having to consciously control each movement.

60. I am able to trust my body to perform skills in competition.

61. I relax myself before competition to get ready to perform.

62. In competition, I am sufficiently prepared to be able to perform on automatic pilot.

63. I can get myself "up” if I feel flat at a competition.

64. Loud noises during competition would not affect my performance.

65. My practice performance suffers when something upsets me at training

66. I use workouts to practise relaxing.

67. Environmental conditions like weather and temperature affect my performance in competitions.

68. I can get my intensity levels just right for practice.

$\begin{array}{lllll}1 & 2 & 3 & 4 & 5 \\ 1 & 2 & 3 & 4 & 5 \\ 1 & 2 & 3 & 4 & 5 \\ 1 & 2 & 3 & 4 & 5 \\ 1 & 2 & 3 & 4 & 5 \\ & & & & \\ 1 & 2 & 3 & 4 & 5\end{array}$


APPENDIX D: Monitoring of Mental Skills Intervention

Please rate the next four statements regarding your use of mental skills during injury rehabilitation IN THE PAST FEW DAYS (SINCE YOU LAST FILLED OUT A JOURNAL) on a scale from 1 to 10 according to the following:

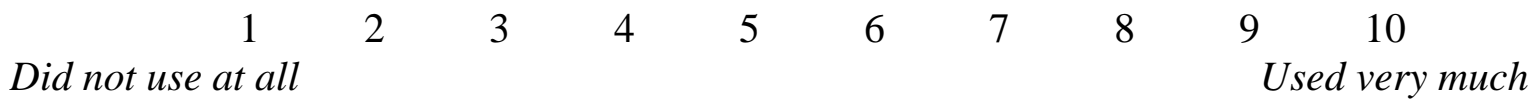

1. How much did you use goal setting in relation to your injury rehabilitation?

2. How much did you use relaxation in relation to your injury rehabilitation?

3. How much did you use imagery in relation to your injury rehabilitation?

4. How much did you manage your self-talk in relation to your injury rehabilitation?

Please rate the next four statements regarding the effectiveness of mental skills on your injury rehabilitation IN THE PAST FEW DAYS (SINCE YOU LAST FILLED OUT A JOURNAL) on a scale from -5 to +5 according to the following:

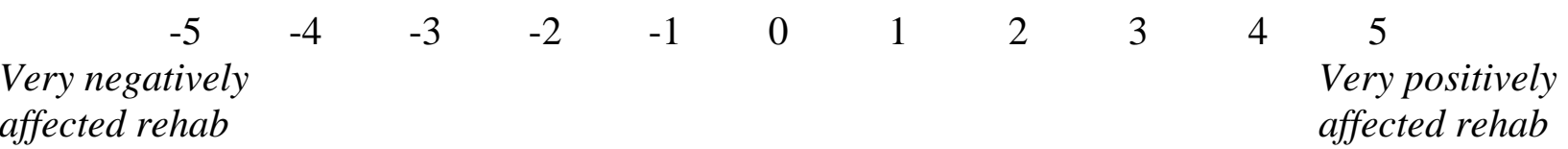

5. How much of an effect did goal setting have on your injury rehabilitation?

6. How much of an effect did relaxation have on your injury rehabilitation?

7. How much of an effect did imagery have on your injury rehabilitation?

8. How much of an effect did self-talk have on your injury rehabilitation?

9. How satisfied were you with the mental skills intervention (goal setting, relaxation, imagery, or self-talk) you learned this week? Answer on a scale of 1 (not satisfied at all) to 10 (extremely satisfied).

(Space was provided for the participant to write comments for all above questions) 
APPENDIX E: Athletic Injury Self-Efficacy Questionnaire

Reprinted with permission from Marcia Milne via e-mail (3/21/07)

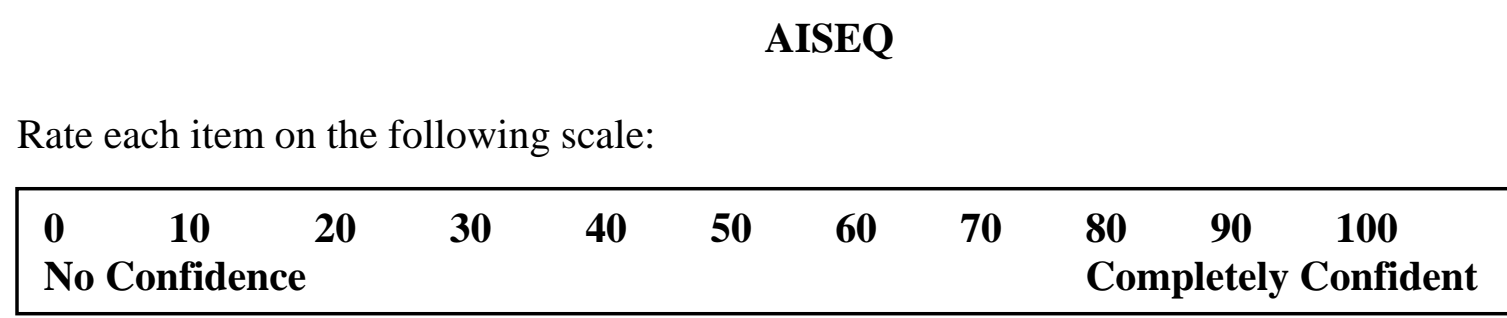

1. I am confident that I can perform all the required rehabilitation exercises.

2. I am confident that I can follow directions from my athletic trainer.

3. I am confident that I can perform all of my rehabilitation exercises correctly.

4. I am confident that I can do my rehabilitation exercises when I am in a bad mood.

5. I am confident that I can do my rehabilitation exercises when I feel I do not have the time.

6. I am confident that I can do my rehabilitation exercises even though I am feeling some discomfort.

7. I am confident that I can follow the rehabilitation schedule (i.e., days and times of rehabilitation) outlined by my athletic trainer. 


\section{APPENDIX F: Modified State Sport Confidence Inventory}

Reprinted with permission from Michelle Magyar via e-mail (4/19/08)

Think about how confident you feel, since your injury, about performing successfully in future competition.

Answer the questions below based on how confident you feel since your injury, about competing in the future. Rate your self-confidence (low, medium, high) on the 100 point scale.

For this questionnaire, success is defined as your ability to perform athletic skills that are specific to your sport in practice or competition as well as you could before your injury.

Rate the confidence you feel since your injury in your ability to...

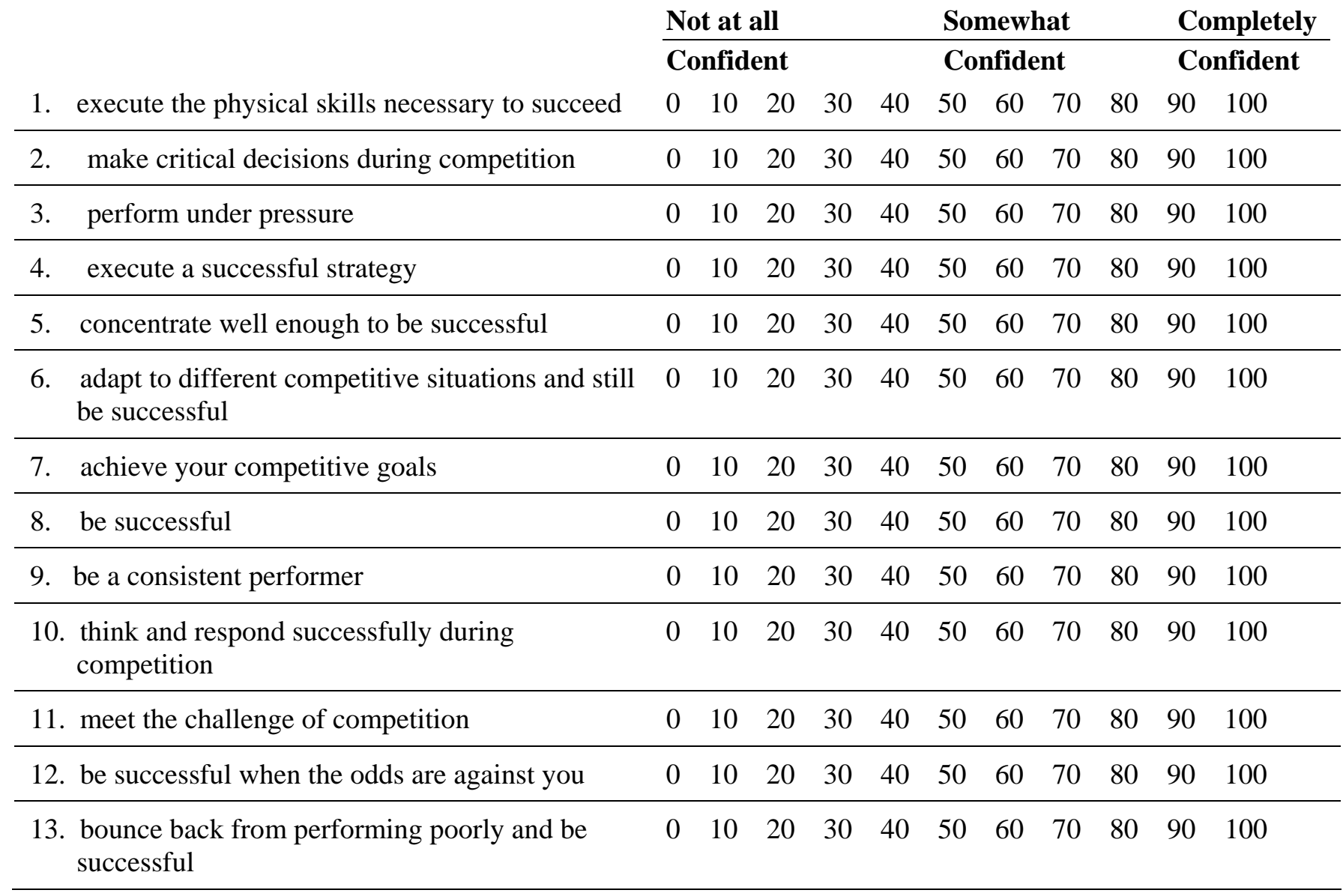




\section{APPENDIX G: Athletic Trainer’s Patient Evaluation Form}

Participant Code Number:

Date:

Directions: Please circle the number that best indicates your assessment of the patient's behavior during their rehabilitation sessions OVER THE PAST FEW DAYS (since you last filled out this form).

1. Circle the number that best indicates the intensity with which this patient has completed rehabilitation exercises.

$$
\begin{array}{lllllll}
\text { Minimum effort } & 1 & 2 & 3 & 4 & 5 & \text { Maximum effort }
\end{array}
$$

2. How frequently has this patient followed your instructions and advice?

$$
\begin{array}{lllllll}
\text { Never } & 1 & 2 & 3 & 4 & 5 & \text { Always }
\end{array}
$$

3. How receptive has this patient been to changes in the rehabilitation program?

$\begin{array}{lllllll}\text { Not very receptive } & 1 & 2 & 3 & 4 & 5 & \text { Very receptive }\end{array}$

4. How would you rate your patient's attitude toward performing his/her rehabilitation exercises?

$\begin{array}{lllllllll}\text { Very negative } & 1 & 2 & 3 & 4 & 5 & 6 & 7 & \text { Very positive }\end{array}$

Directions: Please circle the number that best indicates the rate of recovery of your patient at the current time compared to an average patient with the same injury.

5. Using your clinical judgment, how quickly is this patient recovering compared to an average person with the same injury?

$\begin{array}{lllllll}\text { Significantly below average } & 1 & 2 & 3 & 4 & 5 & \text { Significantly above average }\end{array}$

COMMENTS regarding your patient's behavior in rehabilitation sessions: (Optional)

*The first three items of this measure are from the Sport Injury Rehabilitation Adherence Survey (SIRAS; Brewer et al., 2000). The fourth item is adapted from Levy, Polman, \& Clough (2008). 


\section{APPENDIX H: Athlete-rated Adherence and Attitude Items}

Answer the following questions based on THE PAST FEW DAYS (since you last filled out a journal) in rehabilitation.

1. Rate the intensity with which you have completed your rehabilitation exercises.

$$
\begin{array}{lllllll}
\text { Minimum effort } & 1 & 2 & 3 & 4 & 5 & \text { Maximum effort }
\end{array}
$$

2. How frequently have you followed your athletic trainer's instructions and advice?

$\begin{array}{lllllll}\text { Never } & 1 & 2 & 3 & 4 & 5 & \text { Always }\end{array}$

3. How receptive have you been to changes in the rehabilitation program?

$\begin{array}{lllllll}\text { Not very receptive } & 1 & 2 & 3 & 4 & 5 & \text { Very receptive }\end{array}$

4. How would you rate your attitude toward performing your rehabilitation exercises?

$\begin{array}{lllllllll}\text { Very negative } & 1 & 2 & 3 & 4 & 5 & 6 & 7 & \text { Very positive }\end{array}$




\section{APPENDIX I: Exit Interview}

During the study, you learned four mental skills.

1. Please rank the material you learned from 1 (liked the most) to 4 (liked the least).

Goal Setting Relaxation Self-talk Imagery

2. Now rank the skills according to their usefulness for you in the future from 1 (will probably use the most) to 4 (will probably use the least).

Goal Setting

Relaxation
Self-talk

Imagery

3. What were your impressions of the goal setting unit?

a. Prompts - likes/dislikes

b. Follow ups: How did you use goal setting over the past few weeks?

c. How might you use goal setting in the future?

d. What effects, if any, did goal setting have on your recovery from injury?

i. Prompts - physical or psychological, positive or negative

4. What were your impressions of the self-talk unit?

a. Prompts - likes/dislikes

b. Follow ups: How did you use self-talk over the past few weeks?

c. How might you use self-talk in the future?

d. What effects, if any, did self-talk have on your recovery from injury?

i. Prompts - physical or psychological, positive or negative 
5. What were your impressions of the relaxation unit?
a. Prompts - likes/dislikes
b. Follow ups: How did you use relaxation over the past few weeks?
c. How might you use relaxation in the future?
d. What effects, if any, did relaxation have on your recovery from injury?
i. Prompts - physical or psychological, positive or negative

6. What were your impressions of the imagery unit?
a. Prompts - likes/dislikes
b. Follow ups: How did you use imagery over the past few weeks?
c. How might you use imagery in the future?
d. What effects, if any, did imagery have on your recovery from injury?
i. Prompts - physical or psychological, positive or negative

7. What stood out to you the most from the interventions?

8. How has rehabilitation been for you so far?

a. Follow up - How does it compare to your initial expectations?

9. Is there anything else you would like to share about the intervention or rehabilitation experiences that you have not mentioned already? 


\section{APPENDIX J: Pilot Research}

A pilot study was conducted prior to the proposal of the dissertation with two participants so that the researcher could become familiar with the intervention procedures and determine if any modifications were necessary.

\section{Procedures}

Institutional Review Board approval was obtained prior to conducting the study. Each participant met with the researcher three times over a period of 2-3 weeks. During the first meeting, participants signed a letter of informed consent, completed the TOPS-2, and completed an initial interview similar to the one that will be used in the present study. They then learned the first of two mental skills (either goal setting or relaxation). During the second meeting, participants were interviewed regarding their experience with the previous week’s mental skill. They then learned the second mental skill (either self-talk or imagery). In the third and final meeting, participants were interviewed about their experience with the previous week's mental skill and completed a final interview regarding the entire intervention process. Participants were also asked to complete several online journal assessments throughout the study, which were similar to those that will be used in the present study. Results and Analysis

The process of data analysis included reading through transcripts and coding them according to categories. Data was then reorganized into segments grouped by the categories. Pertinent themes were extracted to compose the following summary of data. Document analysis was conducted to confirm and add to the data from the transcripts. Cases will be presented individually (one participant will be called “Mr. Quad” and one will be called “Mr. Shoulder” in line with their injuries), and then cross case comparisons will be made. 
Mr. Quad. Mr. Quad is a 20 year old sophomore collegiate soccer player. He had been playing soccer for 15 years. His current injury was a strained quadriceps, which he was rehabbing for the second time in a few months (re-injured about a week prior to the study) and had approximately 1.5 months left to go in rehab. He had been attending rehab about 2-3 times a day for 5-6 days per week. His experience with sport psychology included having a sport psychology consultant work with his team (as a group and individually). He stated that he did not receive any formal individualized mental skills training. From examining his scores on the TOPS-2, it appeared that he used goal setting and self-talk more than relaxation and imagery in practice and competition. During the intervention, he said that he had previously used goal setting in sport by setting long-term goals, some goals for practice, and the goal of "getting back as fast as possible” for rehab. He also said that he had previously used self-talk during soccer by being able to get thoughts out of his head (both positive and negative) to move on to presentcentered thoughts. He said he used self-talk in rehabilitation to try to keep a positive attitude.

Mr. Quad learned the skills of goal setting and self-talk. He liked that goal setting gave him a different way to approach things. For the week following learning this skill, he said that he set goals every day and his log confirmed that he did set daily goals for rehab exercises and attitude (rated on a scale of 1 to 10) for each day of the week. The thing that stood out to him from the whole intervention process was setting short-term goals in addition to the long-term goals. He felt that this improved his attitude and helped motivate him. This was exemplified by the quotes, “...instead of looking at the small picture, like you know, what can happen in a month, like you kinda wanna go, what can I do tomorrow to get better?” and “instead of just being like 'oh it’s another boring practice,' it’s like 'got something to do today.'” He also 
thought about the daily goals as flexible and adjustable - if he did not accomplish his daily goal, that was OK, and he would adjust his goals accordingly. Mr. Quad felt that he could apply this short-term goal setting in the future when he returns to sport.

What he liked about managing self-talk was that it is something that could be used all the time, in rehab and many other situations. For instance, he said that he could see himself using this skill (as well as goal setting) for a job in the future. He said that self-talk helped him go into rehab with a better attitude. For example, he said, "like telling yourself that you know it's gonna benefit you...even though you kinda go in not wanting to be there.” Mr. Quad said that he did not do very much rehab the previous week (due to the end of the semester) so he did not get to practice self-talk very much. His log confirmed this in that he only recorded one day of his selftalk use.

Overall, Mr. Quad felt that both goal setting and self-talk helped him have a more positive outlook and attitude in rehabilitation. He liked the structure of the sessions - that I taught the lesson first and then had him practice the skill with exercises. He also liked the use of examples throughout the lesson (such as examples of negative self-talk changed to positive selftalk). He said, “it’s like interaction I thought that was the best part.” In terms of the online survey, he liked that it was short and said it took him about 5 minutes to complete. He did feel that it got repetitive to fill out. Mr. Quad filled out the survey 100\% of the time it was e-mailed to him, although I noticed that he "backdated” by filling out two in one day (filled out that day plus the previous day's in one shot, but filled in different dates on them). Mr. Quad did not offer any suggestions for improvement of the intervention, and in fact, every time I asked him for suggestions, he said a positive instead, like “no, I liked it, it was pretty good I thought...” 
Mr. Shoulder. Mr. Shoulder is a 19 year old freshman collegiate wrestler. He had been wrestling for 13 years. He had shoulder surgery for a torn labrum and had been rehabbing for 4 months, with 2-4 months left to go. He had exposure to sport psychology in that a sport psychologist worked with his team and with him as an individual. He stated that the individual sessions were more counseling-related (he was referred by his coach) and did not include any formal individualized mental skills training. He also did a report in high school on sport psychology so he had some background about the field and what mental skills were. According to the TOPS-2, he showed moderate use of mental skills in practice and competition (the average response was a rating of " 3 ", or "sometimes"), with imagery being the skill he used the most. During the interventions, he stated that he previously used relaxation in yoga class and learned progressive muscle relaxation in a team session with a sport psychologist. He had used imagery before to imagine success in wrestling; he used this before going out on the mat before every match and occasionally at practice. He had not used imagery in rehab thus far.

Mr. Shoulder learned the mental skills of relaxation and imagery. He liked that the relaxation was useful for him in different situations. He learned both diaphragmatic breathing and passive relaxation. He said that he used one or both of these skills every day of the week, and his log confirmed this. He used deep breathing when he was feeling pain during rehabilitation and used both passive relaxation and deep breathing before bed. He listened to the CD with the passive relaxation script on it three times during the week. He thought that deep breathing helped reduce pain and passive relaxation helped him fall asleep (this was also indicated in his log). What stood out to him the most from the whole intervention was that relaxation helped him with sleep. 
For imagery, he liked learning the three different types of imagery (soothing, rehabilitation, and performance). He said that he only went to rehabilitation once during the week due to illness, but he did use rehabilitation imagery (imagining himself doing the exercises) while he was there. He also used soothing imagery (imagining his "happy place”) twice while he was sick, although he did not record this in his log. In the future, Mr. Shoulder said he could see himself using the rehabilitation and performance imagery the most because they apply directly to his sport. He thinks he will make performance imagery part of his pre-competition routine, and could see himself using soothing imagery in stressful and painful situations.

Overall, Mr. Shoulder liked that both of the skills he learned were useful in many different situations. He found that both skills were useful for dealing with his mood, pain, and discomfort during rehab. He saw the value in practicing the skills. He said, "I got better with deep breathing the more I did it" and "I think the more I do the two exercises, the better I'll get at them and the more it will help.” He was hopeful that using the skills would help him recover faster by saying, "hopefully it does help with making you recover faster from your injury and that's what I'm waiting for, to see if these kinda help me along.”

Mr. Shoulder felt that the intervention sessions were “well planned and organized.” He liked that he has the relaxation CD to keep and thought the logs were helpful in making the skills more part of his routine. He mentioned a couple times that he liked the clip-art in the manual. He also liked that I was teaching him skills as if he was a student in my class and that the sessions were one-on-one. He said, "I just think doing it like this is a lot better than reading it from a book or hearing it on tape...I just think it just sticks in your mind more and if I have questions you're there to ask...” 
He thought that the online surveys were a bit long and took him about 10 minutes to fill out. He was confused about some of the directions so I explained this to him after our second session and then he understood what to do. He also felt that the questions were repetitive, but assumed there was a purpose for this. He did like the option of a comment box under some of the questions, although he only used these once. He completed the survey only $50 \%$ of the times I emailed him the link; however, he did mention that he was ill during most of the second week, so perhaps this was a reason he did not fill them out at that time. Mr. Shoulder did have some suggestions regarding the logs in the manuals (to include both date and time of day that the skill is used) and for imagery (to have props in the session to make the imagery more vivid). He also felt that the song I included on the relaxation CD was a bit long, but a good choice for music.

In terms of their injury experiences, both participants had negative thoughts about the injury and their long-term rehabilitation, but also a positive outlook on returning to play - that they were doing what was necessary to get better. They also both put a lot of time into rehab by being there for many hours and most days of the week. Both of them stayed involved with their teams by attending practices and doing rehabilitation on the sidelines. They said they saw their coaches and teammates every day during rehabilitation.

Cross-case comparison. Both participants had favorable impressions of the intervention process as a whole. They liked the interactive component of the sessions. They also both liked that the skills they learned were useful in a variety of situations - rehab, home, sport, and school. One difference between the two participants was in the perceived benefits of the skills. Mr. Quad perceived only psychological benefits (improved attitude and outlook in rehabilitation, increased motivation), while Mr. Shoulder perceived both physical (less pain and discomfort) and psychological (improved mood) benefits. When asked if the intervention had any physical 
benefit, Mr. Quad said that he did not experience this. Finally, both participants were fairly compliant with the intervention, in that they showed up to all the meetings and brought their materials with them; however, Mr. Quad was more compliant with filling out the online surveys. The lack of suggestions by both participants in the study may have been due to the fact that the researcher was conducting both the interventions and interviews; therefore, the participants may have responded in a socially desirable manner.

The results of this pilot research informed the present study in that some of the intervention and assessment materials were slightly altered. The procedures for administering the online journals will also be modified for the present study (e.g., participants e-mailed at the same time each day, obtaining e-mails from Simple Forms when participants complete the survey to detect when participants actually fill them out). To account for the possibility of socially desirable responses by participants, a research assistant will do the post-intervention interview in the present study. In addition to the pilot research, a colleague who is experienced in the fields of both sport psychology and athletic training reviewed the materials and offered suggestions that were taken into consideration when creating the final materials. 
APPENDIX K: Approval from Institutional Review Board

The following new IRB Protocol has been approved.

Tracking \#: H-21058

PI : Etzel, Edward

Title: An individualized multimodal mental skills intervention for college athletes undergoing injury rehabilitation

Approval Date: 08/06/2008

Expiration Date: 08/05/2009

(Sent via e-mail on $8 / 6 / 08$ )

The following Amendment has been approved.

Tracking \#: AMEND-1175 (H-21058)

PI : Etzel, Edward

Title: An individualized multimodal mental skills intervention for college athletes undergoing injury rehabilitation

(Sent via e-mail on 8/12/08) 


\title{
APPENDIX L: Informed Consent Form for Athletes
}

\section{W- WestVirginiaUniversity. Office of Research Compliance \\ CONSENT AND INFORMATION FORM}

OMR ICF (Athlete - Nonpaid)

\author{
Principal Investigator: Etzel, Edward \\ Department: Physical Education \\ Tracking Number: $\quad \mathrm{H}-21058$
}

\section{Study Title:}

An individualized multimodal mental skills intervention for college athletes undergoing injury rehabilitation

\section{Co-Investigator(s):}

Shapiro, Jamie,

\section{Sponsor}

\section{Contact Persons}

In the event you experience any side effects or injury related to this research, you should contact Jamie Shapiro at 732-239-2888. If you have any questions, concerns, or complaints about this research, you can contact Jamie Shapiro at 732-239-2888 or Dr. Ed Etzel at 304-293 $-7062$

For information regarding your rights as a research subject, you may contact the Office of Research Compliance at 304/293-7073.

Introduction

You, , have been asked to participate in this research study, which has been explained to you by Jamie Shapiro, M.S. This study is being conducted by Jamie Shapiro, M.S. in the College of Physical Activity and Sport Sciences at West Virginia University. This

\begin{tabular}{llcl}
\hline Tracking \#: & $H-21058$ & Page 1 of 5 \\
Approved On: & $08 / 06 / 2008$ & \\
Valid Through: & $08 / 05 / 2009$ & \\
Last Amended: & $8 / 12 / 2008$ &
\end{tabular}

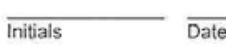


Tracking \#: $\quad \mathrm{H}-21058$

research is being conducted to fulfill the requirements for a doctoral dissertation in Sport and Exercise Psychology in the College of Physical Activity and Sport Sciences at West Virginia University, under the supervision of Dr. Ed Etzel.

\section{Purposes of the Study}

The purpose of this study is to learn more about injured athletes ' experiences with a sport psychology mental skills intervention. WVU expects to enroll approximately five subjects; a total of approximately five subjects at all sites are expected to participate in this study.

\section{Description of Procedures}

This study involves learning sport psychology mental skills, filling out surveys online, and being interviewed about the experience and will take approximately 8 hours over an 8 week period for you to complete. During the first meeting, you will be asked to fill out a questionnaire regarding your use of mental skills. This will take approximately 15 minutes. You will then complete an interview lasting about 15 minutes. Throughout the study you will be asked to complete an online journal regarding your use of mental skills in rehabilitation, confidence in rehabilitation and return to sport, your attitude and effort in rehabilitation, and satisfaction with the mental skills you learn. This will take approximately 10 minutes. In the final meeting, you will complete an interview which will take approximately 30 minutes. For all the questionnaires and interviews, you do not have to answer all the questions. You will have the opportunity to see the questionnaires before signing this consent form. All interviews will be audio taped.

\section{Risks and Discomforts}

There are no known or expected risks from participating in this study, except for the mild frustration associated with answering the questions.

\section{Alternatives}

You do not have to participate in this study.

\begin{tabular}{lllll}
\hline Tracking \#: & $\mathrm{H}-21058$ & Page 2 of 5 & \\
Approved On: & $08 / 06 / 2008$ & & Initials & \\
Valid Through: & $08 / 05 / 2009$ & & \\
Last Amended: & $8 / 12 / 2008$ & &
\end{tabular}


Tracking \#: $\quad \mathrm{H}-21058$

You do not have to participate in this study. At this time, there are no alternatives that could be considered in your case.

\section{Benefits}

You may not receive any direct benefit from this study, however it is possible that the skills you learn will benefit your recovery from injury and your sport performance. The knowledge gained from this study may eventually benefit others.

\section{Financial Considerations}

There will be no cost to participate in this study. You will not receive any tangible payments for participating in this study.

\section{Confidentiality}

Any information about you that is obtained as a result of your participation in this research will be kept as confidential as legally possible. Your research records and test results, just like hospital records, may be subpoenaed by court order or may be inspected by federal regulatory authorities without your additional consent. In addition, there are certain instances where the researcher is legally required to give information to the appropriate authorities. These would include mandatory reporting of infectious diseases, mandatory reporting of information about behavior that is imminently dangerous to you or to others, such as suicide, child abuse, etc. Audiotapes will be kept locked up and will be destroyed as soon as possible after the research is finished. In any publications that result from this research, neither your name nor any information from which you might be identified will be published without your consent.

\section{Voluntary Participation}

Participation in this study is voluntary. You are free to withdraw your consent to participate in this study at any time. Refusal to participate or withdrawal will not affect your future care, your status on your athletic team at your university, and will involve no penalty to you. In the event new information becomes available that may affect your willingness to participate in this study, this information will be given to you so that you can make an informed decision about whether or not to continue your participation. As part of this study, you will be asked to fill out 3 online

\begin{tabular}{lllll}
\hline Tracking \#: & $\mathrm{H}-21058$ & Page 3 of 5 & \\
Approved On: & $08 / 06 / 2008$ & & & \\
Valid Through: & $08 / 05 / 2009$ & & & \\
Last Amended: & $8 / 12 / 2008$ & & &
\end{tabular}


Mental Skills Intervention 134

Tracking \#: $\quad \mathrm{H}-21058$

journals per week. If you fill out less than 2 journals per week, you will be removed from the study. Also, if data collection is interrupted for greater than 7 days (e.g., due to illness or school break), you will be removed from the study. You have been given the opportunity to ask questions about the research, and you have received answers concerning areas you did not understand.

$\begin{array}{lll}\text { Tracking \#: } & \mathrm{H}-21058 & \text { Page } 4 \text { of } 5 \\ \text { Approved On: } & 08 / 06 / 2008 & \\ \text { Valid Through: } & 08 / 05 / 2009 & \\ \text { Last Amended: } & 8 / 12 / 2008 & \end{array}$


Mental Skills Intervention 135

Tracking \#: $\quad \mathrm{H}-21058$

Upon signing this form, you will receive a copy.

I willingly consent to participate in this research.

Signature of Subject or

Printed Name

Date

Time

Subjects Legal Representative

The participant has had the opportunity to have questions addressed. The participant willingly agrees to be in the study.

Signature of Investigator or

Printed Name

Date

Co-Investigator

Tracking \#: $\quad \mathrm{H}-21058$

Approved On: $\quad$ 08/06/2008

Valid Through: $\quad$ 08/05/2009

Last Amended: $\quad 8 / 12 / 2008$
Page 5 of 5

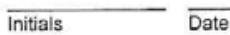




\title{
APPENDIX M: Informed Consent Form for Athletic Trainers
}

\section{N-West VirginiaUniversity. Office of Research Compliance \\ CONSENT AND INFORMATION FORM}

\author{
OMR ICF (ATC)
}

\author{
Principal Investigator: Etzel, Edward \\ Department: Physical Education \\ Tracking Number: $\quad \mathrm{H}-21058$
}

\section{Study Title:}

An individualized multimodal mental skills intervention for college athletes undergoing injury rehabilitation

\section{Co-Investigator(s):}

Shapiro, Jamie

Sponsor

\section{Contact Persons}

If you have any questions, concerns, or complaints about this research, you can contact Jamie Shapiro at 732-239-2888 or Dr. Ed Etzel at 304-293 $-7062$

For information regarding your rights as a research subject, you may contact the Office of Research Compliance at 304/293-7073.

Introduction

You, , have been asked to participate in this research study, which has been explained to you by Jamie Shapiro, M.S. This study is being conducted by Jamie Shapiro, M.S. in the College of Physical Activity and Sport Sciences at West Virginia University. This research is being conducted to fulfill the requirements for a doctoral dissertation in Sport and Exercise Psychology in the College of Physical Activity and Sport Sciences at West Virginia University, under the

\begin{tabular}{lll}
\hline Tracking \#: & $\mathrm{H}-21058$ & Page 1 of 4 \\
Approved On: & $08 / 06 / 2008$ & \\
Valid Through: & $08 / 05 / 2009$ & \\
Last Amended: & $8 / 12 / 2008$ &
\end{tabular}


Tracking \#: $\quad \mathrm{H}-21058$

supervision of Dr. Ed Etzel, Ed.D.

\section{Purposes of the Study}

The purpose of this study is to learn more about injured athletes ' experiences with a sport psychology mental skills intervention. WVU expects to enroll approximately five subjects; a total of approximately five subjects at all sites are expected to participate in this study.

\section{Description of Procedures}

For you, the Athletic Trainer (ATC) working with a participant in this study, this study involves completing an evaluation of the participant's progress in rehabilitation and will take approximately 1 hour over an 8 week period for you to complete. You will be asked to fill out a questionnaire regarding your athlete's adherence, attitude, and recovery time in rehabilitation 3 times per week. This will take approximately 2 minutes to complete. You do not have to answer all the questions. You will have the opportunity to see the questionnaire before signing this consent form.

\section{Risks and Discomforts}

There are no known or expected risks from participating in this study, except for the mild frustration associated with answering the questions.

\section{Alternatives}

You do not have to participate in this study.

At this time, there are no alternatives that could be considered in your case.

\section{Benefits}

You may not receive any direct benefit from this study. The knowledge gained from this study may eventually benefit others.

\section{Financial Considerations}

There is no cost to participate in this study, and you will not receive any payment or class credit for participating.

\begin{tabular}{lll}
\hline Tracking \#: & $\mathrm{H}-21058$ & Page 2 of 4 \\
Approved On: & $08 / 06 / 2008$ & \\
Valid Through: & $08 / 05 / 2009$ & \\
Last Amended: & $8 / 12 / 2008$ &
\end{tabular}

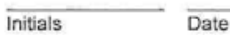




\section{Confidentiality}

Any information about you that is obtained as a result of your participation in this research will be kept as confidential as legally possible. Your research records and test results, just like hospital records, may be subpoenaed by court order or may be inspected by federal regulatory authorities without your additional consent. In any publications that result from this research, neither your name nor any information from which you might be identified will be published without your consent.

\section{Voluntary Participation}

Participation in this study is voluntary. You are free to withdraw your consent to participate in this study at any time. Refusal to participate or withdrawal will not affect your employee status at West Virginia University or your class standing or grades, and will involve no penalty to you. In the event new information becomes available that may affect your willingness to participate in this study, this information will be given to you so that you can make an informed decision about whether or not to continue your participation. You have been given the opportunity to ask questions about the research, and you have received answers concerning areas you did not understand.

\begin{tabular}{lll}
\hline Tracking \#: & $\mathrm{H}-21058$ & Page 3 of 4 \\
Approved On: & $08 / 06 / 2008$ & \\
Valid Through: & $08 / 05 / 2009$ & \\
Last Amended: & $8 / 12 / 2008$ &
\end{tabular}


Mental Skills Intervention 139

Tracking \#: $\quad$ H-21058

Upon signing this form, you will receive a copy.

I willingly consent to participate in this research.

Signature of Subject or Printed Name

Date

Subjects Legal Representative

The participant has had the opportunity to have questions addressed. The participant willingly agrees to be in the study.

Signature of Investigator or

Printed Name

Date

Time

Co-Investigator

Tracking \#: $\quad \mathrm{H}-21058$

Approved On: $\quad$ 08/06/2008

Valid Through: $\quad$ 08/05/2009

Last Amended: $\quad 8 / 12 / 2008$
Page 4 of 4

Initials

Date 


\section{APPENDIX N: Self-ranking of Mental Skills Use}

Participant Code Number:

Please rank your current use of the following mental skills in your sport, with “ 1 ” being the skill you use the most and " 4 ” being the skill you use the least. The definitions of the four mental skills below are as follows.

Goal setting: You frequently set both long- and short-term specific goals for yourself in your sport and adjust these goals as necessary.

Relaxation: If you feel yourself getting anxious, you calm yourself down by using deep breathing or a relaxation technique, such as progressive muscle relaxation.

Imagery: You are able to get a vivid and controllable image of yourself performing in your head.

Self-talk: The statements you say to yourself are useful towards your performance. If you find that your self-talk is not useful or negative, you have the ability to change those thoughts into more productive thoughts for performance.

Please rank your use of each of these skills from 1 (use the most) to 4 (use the least). Goal Setting

Relaxation Imagery Self-talk 


\section{APPENDIX O: Sample Outlines of Mental Skills Interventions}

\section{Goal Setting}

- If applicable, discuss previous intervention (questions, concerns, issues) (5-10 min)

- Ask participant: What is your experience with setting goals? In sport? In rehabilitation?

- Ask participant a current goal they have for rehabilitation. Use this example while teaching the skill.

- Explain purposes for goal setting in rehabilitation: motivation, focus, increasing effort, tracking progress

- Definitions of goals: outcome, performance, process

- Principles of goal setting

o For “positively stated” - do exercise, “close your eyes and whatever you do, don’t think of a pink elephant.”

- Fill out goal setting worksheet for rehabilitation. Participant will write on his/her copy and I will write on my copy.

- Explain the goal setting log for daily goals. Fill in the first daily goal with the participant. Explain to the participant that the Athletic Trainer may need to help set goals for rehab.

- Questions from the participant

- Tell participant that I will need him/her to bring back the manual to each meeting and that I will need to copy them for analysis purposes. 


\section{Self-Talk}

- If applicable, discuss previous intervention (questions, concerns, issues) (5-10 min)

- Ask: What has your experience been with monitoring and changing your self-talk?

In sport? Rehab?

- What is self-talk?

- Types of self-talk and effects of each

o For “positively stated” - do exercise, “close your eyes and whatever you do, don’t think of a pink elephant.”

- Have participant write examples of positive and negative self-talk they have used recently

- How to reframe self-talk (thought-stopping technique)

- Written exercise - thought-stopping technique/reframing

Negative statements:

Thought Stopping Cue:

Positive statements:

- Explain affirmation statements and have participant create one

- Explain self-talk log

- Questions from the participant

- Tell participant that I will need him/her to bring back the manual to each meeting and that I will need to copy them for analysis purposes.

\section{Relaxation}

- If applicable, discuss previous intervention (questions, concerns, issues) (5-10 min)

- Ask: What are some techniques you currently use to relax? In sport? Rehab? Home? 
- Rate 1-10 how relaxed you feel:

- Explanation of diaphragmatic breathing: why do it, how to do it, when and where to do it

- Practice diaphragmatic breathing

- Process: What was that like for you?

- Ask: when can deep breathing be useful for you?

- Explanation of passive muscle relaxation: why do it, how to do it, when and where to do it

- Practice passive muscle relaxation

- Process: What was that like for you?

- Ask: when can passive relaxation be useful for you?

- Rate 1-10 how relaxed you feel:

- Provide athlete with CD to use at home

- Explain relaxation log: record date, type of relaxation, comments about the experience

- Questions from the participant

- Tell participant that I will need him/her to bring back the manual to each meeting and that I will need to copy them for analysis purposes.

\section{Imagery}

- If applicable, discuss previous intervention (questions, concerns, issues) (5-10 min)

- Ask: What have your experiences been with imagery? In sport, rehab, home?

- Discuss different types (healing, soothing, rehabilitation, sport performance) and functions

- Explain the two perspectives (internal/external)

- Explain principles of effective imagery

- Practice soothing imagery, process experience 
o Perspective:

o Vividness __ ; Controllability ___

- Practice rehabilitation imagery, process experience

o Perspective:

o Vividness ___ C Controllability ___

- Practice sport performance imagery, process experience

o Perspective:

o Vividness __ _ Controllability __

- Explain how the athlete could create his/her own imagery script.

- A worksheet will be provided for the participant to monitor use of imagery for each day of the week. There will be an explanation of how to use this sheet.

- Questions from the participant

- Tell participant that I will need him/her to bring back the manual to each meeting and that I will need to copy them for analysis purposes. 
Mental Skills Intervention 145

APPENDIX P

Sample Intervention Materials 


\title{
Mental Skills Manual for Injury Recovery
}

\author{
Introduction
}

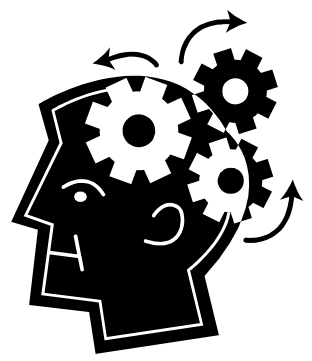

In your sport, you may use sport psychology mental skills such as goal setting, relaxation, imagery, and managing your selftalk in practice and competition. Mental skills can also be used to enhance your recovery from your injury.

\section{Why use mental skills during your rehabilitation from injury?}

Since you cannot fully physically practice, injury recovery is a great time to sharpen your mental skills. Research* has found that the use of mental skills, such as goal setting, relaxation, imagery, and managing self-talk have positive outcomes for injured athletes such as:
$\checkmark$ Improved mood
$\checkmark$ Reduced pain
$\checkmark$ Reduced stress and anxiety
$\checkmark$ Greater confidence for a full recovery
$\checkmark$ Increased sense of control
$\checkmark$ Better adherence to the rehabilitation regimen
$\checkmark$ A faster, more efficient recovery

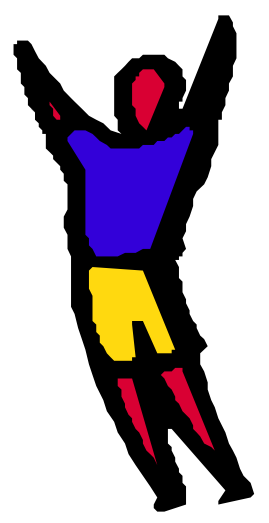

With the use of this manual and meetings with your sport psychology consultant, you will learn how to apply mental skills to your injury rehabilitation. When you return to sport, you can then incorporate these skills into your practices and competitions/games to help reach your optimal performance. 


\section{Goal Setting}

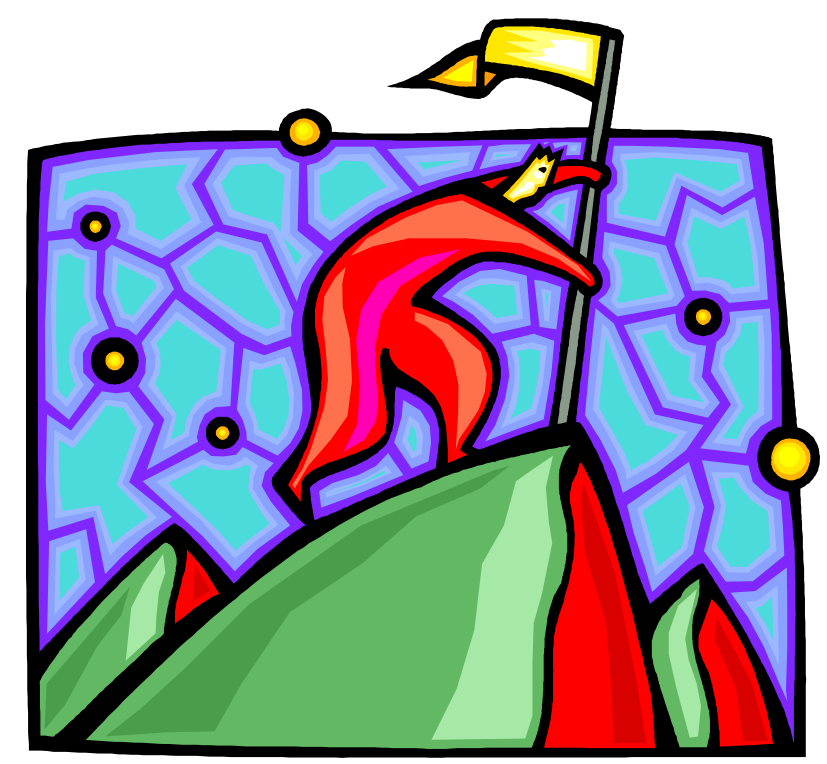

This unit will cover:

- Purposes and benefits of goal setting for rehab

- Types of goals

- Principles of goal setting

- Goal setting worksheets 
Setting both long- and short-term goals for your injury recovery are essential. Many athletes have experience with goal setting from their sport, however, not all athletes know how to set goals correctly. These worksheets will help you set effective goals for your rehabilitation, recovery, and return to sport.

\section{Why use goal setting?}

There are several purposes for goal setting in rehabilitation. These include:

- Motivation

- Focus on what needs to be done

- Increasing effort

- Tracking progress

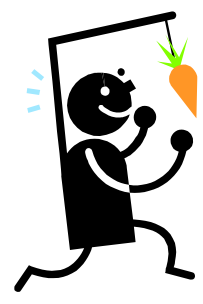

Setting goals can benefit you by making you feel more in CONTROL of your recovery.

\section{Types of Goals}

There are three types of goals you can set for rehabilitation and recovery:

1. Outcome Goals - focus on the end result

$>$ Example: Returning to play

2. Performance Goals - focus on standards to achieve compared to one's own previous performance

D Example: Increasing weight and reps on leg press

3. Process goals - focus on the actions one must execute to perform well

$>$ Example: Icing for $20 \mathrm{~min} / 3$ times per day; Positive self-talk during rehab

While outcome goals are important motivators, performance and process goals are more under the control of the goal setter. For example, returning to play is not always under the control of the athlete - the coach is often times the one who makes the final decision. But improving range of motion, increasing weight on exercises, and keeping a positive attitude are all under the control of the athlete. Performance and process goals have been found to improve rehabilitation outcomes for injured athletes (Evans \& Hardy, 2002; Theodorakis et al., 1997). You should set all three types of goals for your recovery and once you return to sport.

On the next page you will find some principles of effective goal setting to keep in mind while formulating your goals. 


\section{Principles of Effective Goal Setting}

Remember SMARTER goal setting:

Specific

Measurable

Adjustable

Realistic

Time-based

Evvaluated

Recorded (Ink it, just don't think it!)

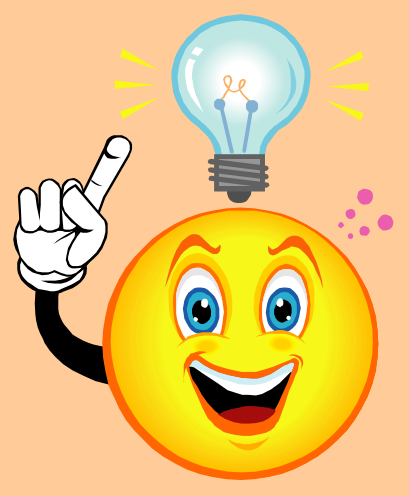

Other things to keep in mind when setting goals:

$\square$ Set both long- and short-term goals

- The short-term goals are especially important for you because the rehab and return-to-sport process may be lengthy.

$\square$ Establish a goal ladder

- Identify the specific behaviors needed to reach your goals and organize them into a logical sequence. Add target dates so you have a time frame in which to achieve your goals.

Goals should be positively stated

- Avoid the words "don't," "not," or "without" in goals. You should state what you will do instead of what you will not do.

\section{Set both physical and mental goals}

- Set goals for both physical and psychological skills (i.e., practice imagery 3 times a day for 15 minutes). 
Date:

\section{Goal Setting Worksheet for Rehabilitation}

1. What is your long-term goal for rehabilitation? When would you like to achieve this goal?

2. What are your goals for this month?

3. What are your goals for this week?

4. What are your goals for your next rehabilitation session? 
5. Who can help you achieve your goals?

\section{Roadblock exercise:}

On the way to recovery, you may encounter some obstacles. List possible roadblocks you might face on the way to reaching your goals.

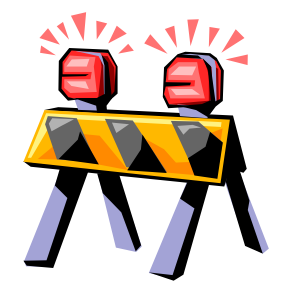

1)

2)

3)

Now think of strategies to deal with these obstacles should you face them. Strategy to overcome Roadblock 1:

Strategy to overcome Roadblock 2:

Strategy to overcome Roadblock 3: 


\section{Goal Setting Log}

Long term goal for recovery:

DAILY Goals for the week of

$$
\text { (dates) }
$$

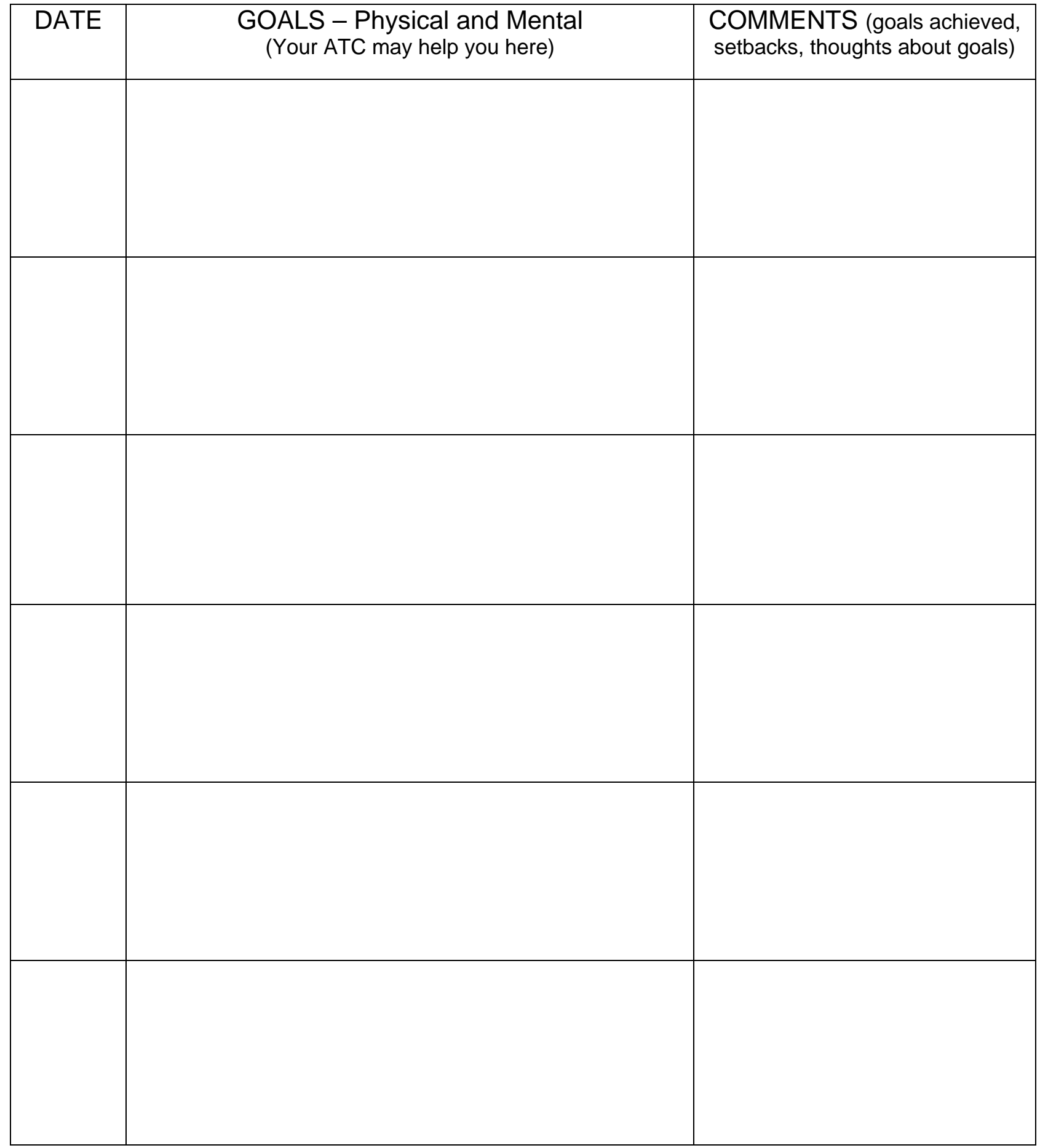




\section{Managing Self-Talk}

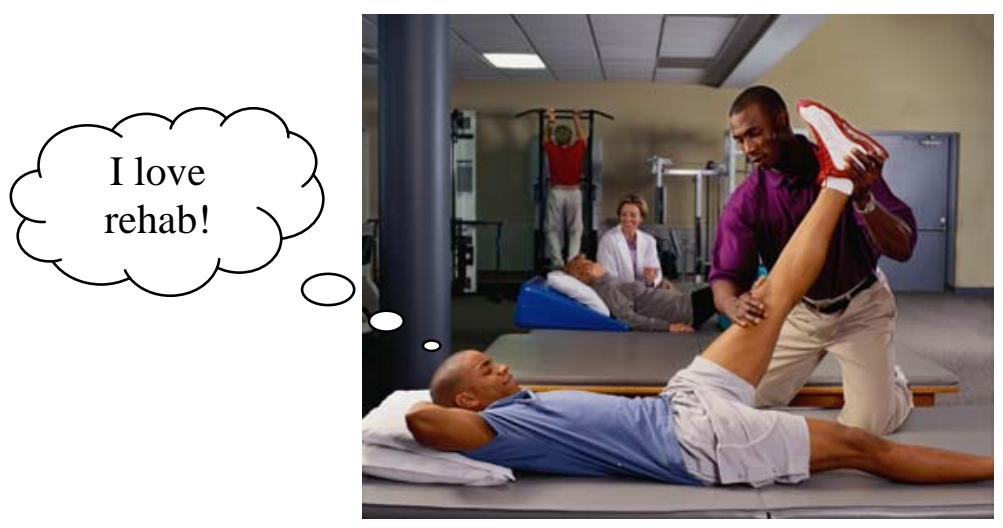

This unit will cover:

- Types of self-talk

- How self-talk could affect you in rehab

- Reframing negative self-talk to positive self-talk

- Affirmation statements 


\section{What is self-talk and how could it affect injury recovery?}

Any time you are thinking, you are essentially talking to yourself. Your thoughts are powerful - they influence your emotions and behaviors (e.g., effort and attitude in rehab). There are three types of self-talk (Weinberg \& Gould, 2007):

1. Positive self-talk: motivational; can help with positive attitude, confidence Examples: "You can do this"; "Your hard work will pay off"

2. Instructional self-talk: tell yourself what to do; keeps you focused on the task at hand

\section{Examples: "Tighten quad"; "Lift and lower your leg slowly"}

3. Negative self-talk: self-defeating and critical, unproductive; can lead to low motivation and negative mood

Examples: "This stinks"; "This is too hard"

$\rightarrow$ In your self-talk, you want to avoid telling yourself "not" to do something (for example, "don't fall", "don't be negative"). Telling yourself "not" to do something can inadvertently trigger it to happen (Janelle, 1999). Therefore, tell yourself what TO DO as opposed to what not to do.

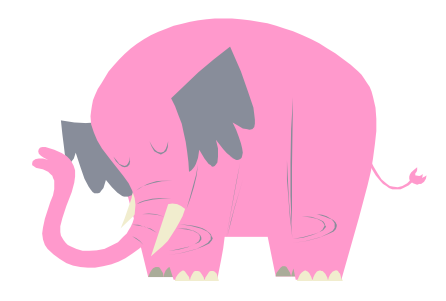

What are some examples of self-talk you have used recently?

Positive:

Negative: 


\section{Reframing Self Talk}

When thoughts are negative and unproductive, you want to change ("reframe") them into more useful, productive thoughts. One technique for reframing self-talk is the thought-stopping technique. Thought stoppage can be accomplished in three steps (Krane \& Greenleaf, 1999):

1. Identify negative self-talk.

2. As soon as the negative statement is said, insert a thought-stopping cue. Examples include:

- Saying the word "STOP!" out loud or to yourself

- Picturing a stop sign in your head

- Snapping a rubber band on your wrist

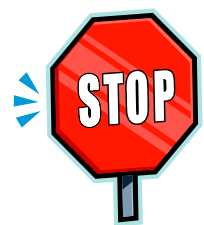

3. Replace the negative statement with a more productive, positive one.

Here is an example of the thought-stopping technique applied to a negative statement an injured athlete might say:

1. Negative statement: "I'll never get back to my skill level before I got hurt."

2. Thought-stopping cue: "STOP!"

3. Positive statement: "Even if I never regain my skills, I can still work hard in rehab so that I can get back and be a valuable contributor to the team."

The following table shows some negative thoughts that an injured athlete might have during rehabilitation and how to reframe these statements to be more positive.

\section{Negative Thoughts}

This hurts too much.

I hate rehab.

This is useless.

I'm too weak.

\section{Positive replacement thoughts}

Be tough; hang in there.

Rehab will make me stronger.

This will get me back to play.

Look how far I've come; I'm getting stronger. 


\section{Self-Talk Worksheet}

What you say to yourself about your injury and rehabilitation may not always be positive. How will you get yourself back on track with what you say and think? Use the following framework:

Negative self-statements (about my injury or rehab):

1.

2.

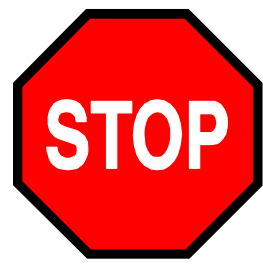
Thought-stopping cue (insert this as soon as you catch yourself saying a negative statement):

Positive self-statements (reframe negative statements from above): 1.

2.

\section{Affirmation Statements}

Write a short statement you could say to yourself during rehab that could motivate you and help you maintain a positive attitude. 


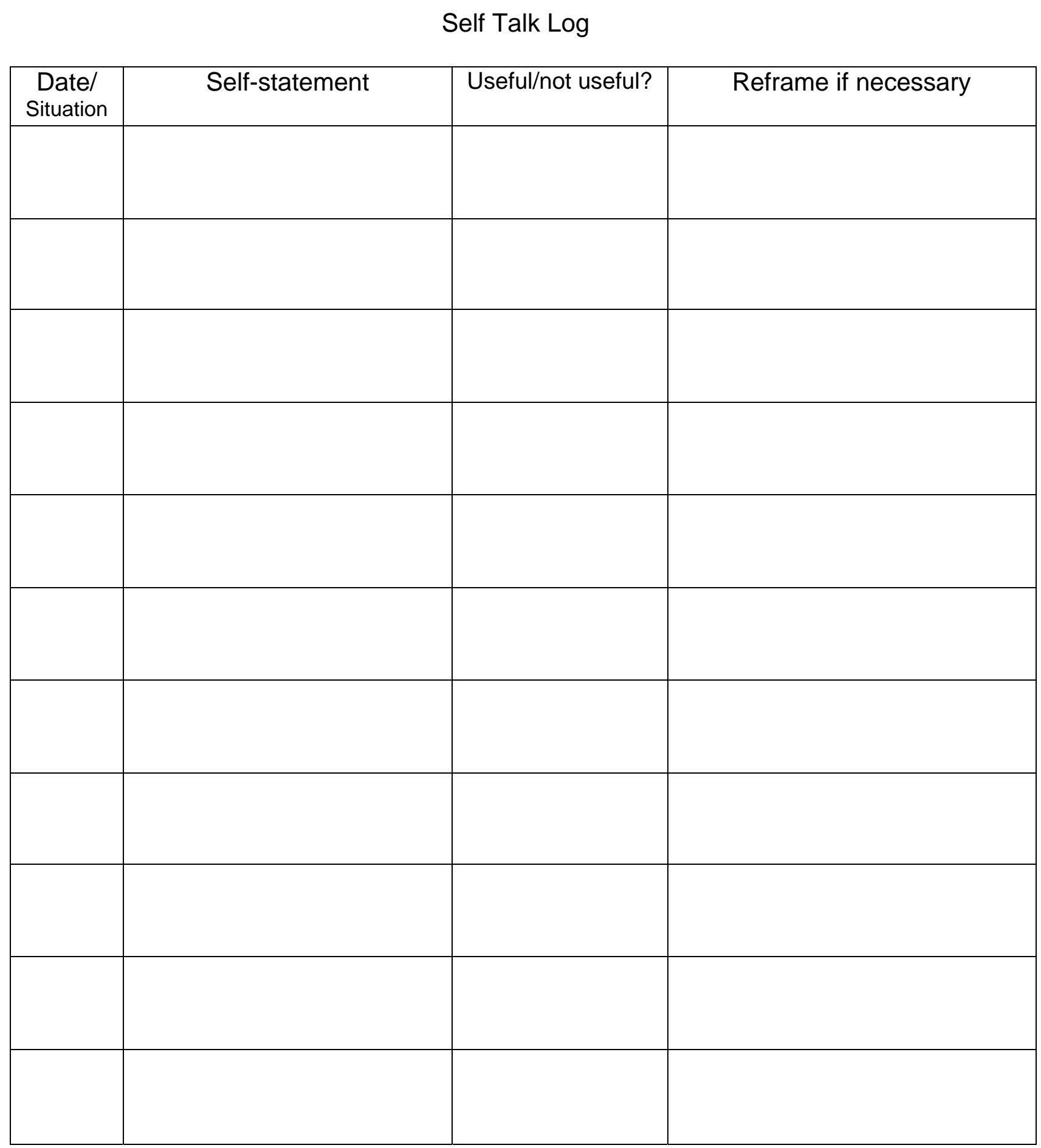




\section{Relaxation}

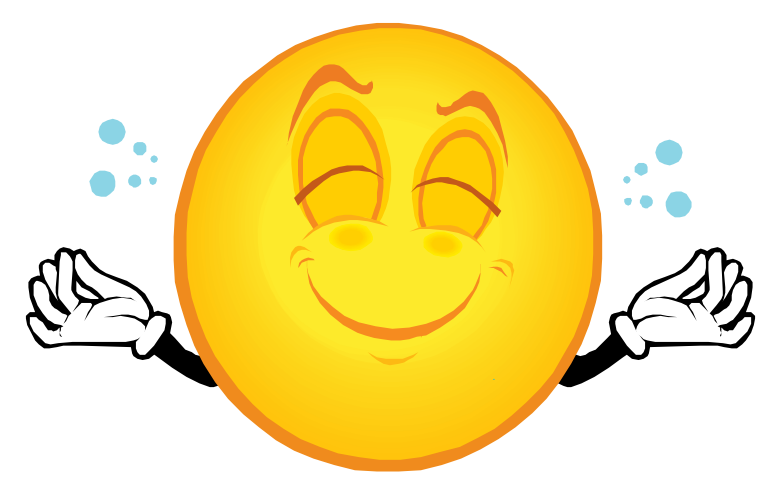

This unit will cover:

- Uses and benefits of relaxation

- Deep breathing technique

- Passive relaxation technique 


\section{Relaxation}

Relaxation is a technique to help you reduce stress and anxiety and improve injury recovery. One study found that a relaxation intervention produced greater muscle strength and lower levels of reinjury anxiety and pain compared to placebo and control groups following knee surgery (Cupal \& Brewer, 2001).

There are many uses and benefits of relaxation, some of which include (Cupal \& Brewer, 2001; Taylor \& Taylor, 1997):
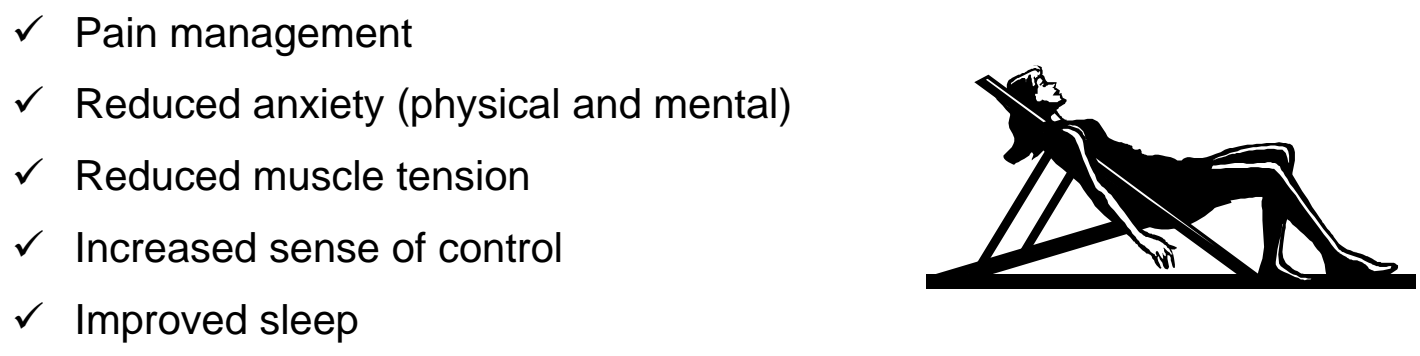

The relaxation techniques that you will learn in this session are:

- Deep breathing

- Passive Relaxation

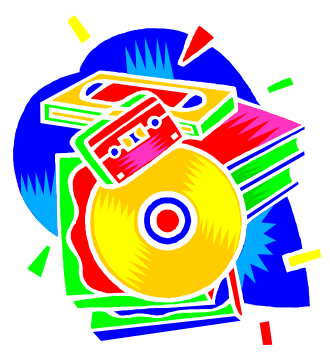

I will take your through both of these exercises. You will then be given a CD to listen to at home. Relaxation is most beneficial when practiced regularly. You should listen to the $C D$ at least 3 times a week to start, and eventually you may be able to relax yourself on your own (without the use of the $C D$ ).

Before we start, rate how relaxed you feel on a scale of 1 to 10 : (not relaxed at all; (extremely relaxed, quite tense)

5

6 


\section{Deep Breathing}

Deep breathing (also called diaphragmatic breathing) is a simple and effective way to decrease muscle tension and anxiety. Deep breathing allows more oxygen to enter the blood stream and reach the muscles, helping the muscles relax better (Taylor \& Taylor, 1997). You could use this technique when your body needs to be relaxed (i.e., stretching), when doing a painful exercise, while receiving treatment, etc.

\section{Instructions for deep breathing}

1. Inhale through your nose.

2. As you inhale, first fill the lower section of your lungs with air by pushing the diaphragm down and forcing the abdomen out.

3. Then fill the middle portion of the lungs by expanding the chest cavity and raising the rib cage.

4. Finally, fill the upper level of the lungs by raising the chest and shoulders slightly.

5. Hold this breath for several seconds.

6. Exhale slowly through the mouth by pulling the abdomen in and lowering the shoulders and chest.

Note: A good idea is to inhale to a count of four and exhale to a count of eight. This ensures that the exhale (more relaxing part) is longer than the inhale. Counting also serves as a distraction (i.e., from pain, discomfort, or negative thoughts).

Adapted from Weinberg \& Gould (2003)

As with any other skill, deep breathing will become easier and more effective with practice. You should practice taking 5-10 deep breaths about 5 times a day. What situations can you think of when deep breathing could be useful for you? (List):

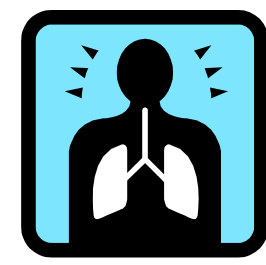




\section{Passive Relaxation}

Passive relaxation includes deep breathing and a focus on tension as it leaves your body (Taylor \& Taylor, 1997). This creates a sense of calmness and general state of physical relaxation. For this exercise, you will imagine tension as a liquid that fills your muscles and then imagine that liquid draining out of your body through plugs on the bottom of your feet.

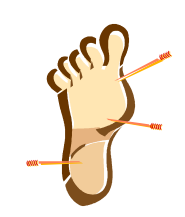

\section{Sample Passive Relaxation Script}

Get into a comfortable position and close your eyes. Imagine that there are drain plugs at the bottom of your feet, and when they are opened, all the tension will drain out of your body and you will become very relaxed. Take a few deep breaths from your diaphragm. Inhale deeply and exhale slowly....inhale, exhale.

Now undo those plugs. Feel the tension begin to drain out of your body...from the top of your head, past your forehead, face, and neck. You are becoming more relaxed. The tension drains out of your jaw and past your neck. Your face and neck are heavy and warm. And you are comfortable. Inhale deeply and exhale slowly.

The tension continues to drain out of your upper body, from your shoulders, down your arms, through your forearms, and your hands. Your shoulders, arms, and hands are now warm and comfortable and you are feeling more and more relaxed.

The tension now drains out of your upper body, past your chest and upper back, down through your stomach and lower back. Now your entire upper body is heavy and warm. And you are feeling relaxed. Inhale, exhale, inhale, exhale.

The tension continues to drain out of your body, past your buttocks and down through your thighs and knees. Your lower body is becoming very relaxed. The tension drains out of your calves and past your ankles, to the balls of your feet and your toes. There is no more tension left in your body. You are completely relaxed. Your whole body feels heavy, warm, comfortable, and relaxed. Now replace the plugs so no more tension can get in. Inhale deeply, and exhale slowly.

Take a few moments to enjoy this peaceful feeling of relaxation. Now, when you are ready, and only when you are ready, open your eyes and return to the room.

Adapted from Taylor \& Taylor (1997)

Now, rate how relaxed you feel on a scale of 1 to 10:

(not relaxed at all;

(extremely relaxed, quite tense)

1

2

3

4

5

6

7

8

little to no tension)

9

10 


\section{Relaxation Log}

Record when you practice relaxation (deep breathing, listening to the relaxation $C D$ ) and your thoughts about the experience.

\begin{tabular}{|l|l|l|}
\hline DATE & $\begin{array}{c}\text { DESCRIPTION OF RELAXATION } \\
\text { (type, setting) }\end{array}$ & COMMENTS \\
\hline & & \\
\hline & & \\
\hline & & \\
\hline & & \\
\hline & & \\
\hline & & \\
\hline & & \\
\hline & & \\
\hline & & \\
\hline & & \\
\hline & & \\
\hline & & \\
\hline & & \\
\hline & & \\
\hline & & \\
\hline & & \\
\hline & & \\
\hline
\end{tabular}




\section{Imagery}

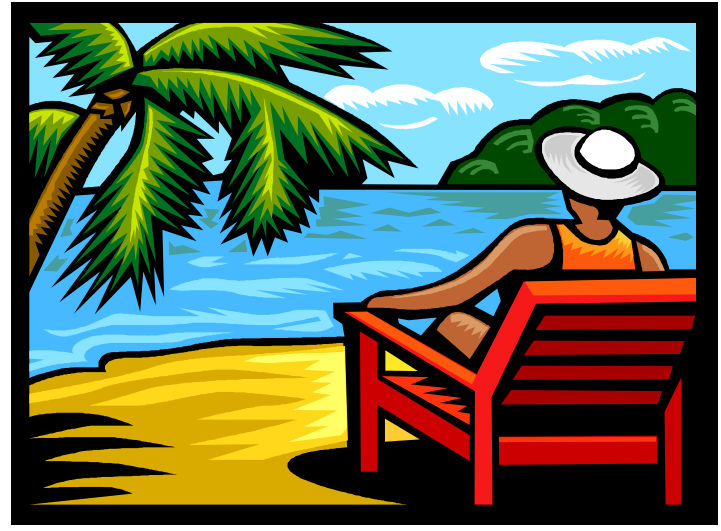

This unit will cover:

- Types and functions of imagery for rehab

- Perspectives of imagery

- Principles of effective imagery

- Imagery exercises

o Soothing

o Rehabilitation

o Performance 
Imagery allows people to recreate experiences as well as create new experiences in their minds (Vealey \& Greenleaf, 2001). There are several different types of imagery that could be useful during injury recovery. Each type may serve a different function in your recovery as seen in the chart below (Taylor \& Taylor, 1997).

\begin{tabular}{|l|l|l|}
\hline \multicolumn{1}{|c|}{ Imagery Type } & \multicolumn{1}{|c|}{ Description } & \multicolumn{1}{c|}{ Function } \\
\hline Soothing & $\begin{array}{l}\text { Picturing yourself in a calm, } \\
\text { relaxing, and comforting place } \\
\text { (e.g., beach) }\end{array}$ & Relaxation, pain management \\
\hline Healing & $\begin{array}{l}\text { Imagining your body mending } \\
\text { the injury and seeing the area } \\
\text { completely healed }\end{array}$ & $\begin{array}{l}\text { Physiological changes that } \\
\text { enhance healing }\end{array}$ \\
\hline Rehabilitation & $\begin{array}{l}\text { Seeing yourself successfully } \\
\text { completing your rehab } \\
\text { exercises }\end{array}$ & $\begin{array}{l}\text { Build confidence in ability to } \\
\text { do exercises }\end{array}$ \\
\hline Performance & $\begin{array}{l}\text { Imagining yourself performing } \\
\text { skills specific to your sport; } \\
\text { seeing yourself be victorious } \\
\text { in competition }\end{array}$ & $\begin{array}{l}\text { Build confidence in ability to } \\
\text { perform sport skills; work on } \\
\text { technique, tactics, mental } \\
\text { preparation, and competitive } \\
\text { performance during rehab }\end{array}$ \\
\hline
\end{tabular}

\section{Two Different Perspectives}

You can use two different viewpoints when doing imagery, an internal or external view.

$>$ Internal Imagery- Viewing the image through your own eyes and from your perspective. For example, if you imagined yourself on a beach, you would be able to see the beach through your own eyes, like you were actually there.

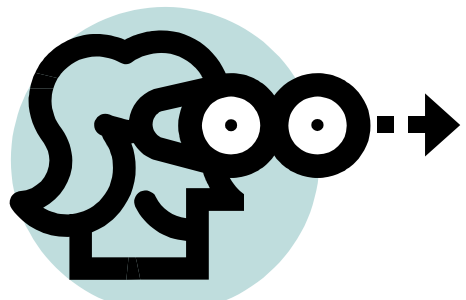

$>$ External Imagery- Viewing the image from an outsider's perspective, as if watching yourself in a movie. For example, with the beach scene, you would see yourself on the beach from a bystander's view.

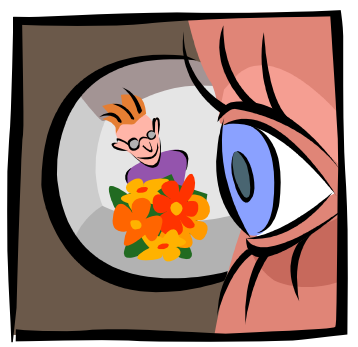




\section{Principles of Effective Imagery}

The keys to effective imagery are vividness and controllability. You want the images to be very clear (vivid) and do what you want them to do (controllability). Both of these improve with PRACTICE! Here are some tips to help you with imagery:

$\checkmark$ Be relaxed (Weinberg \& Gould, 2003). Do some deep breathing and relaxation before beginning your imagery.

$\checkmark$ Use all the senses. The closer the images are to real life, the more vivid and effective they will be. The senses you want to include are:

- Sight

- Sound

- Smell

- Taste

- Touch

- Kinesthetic sense - sensation of body position and movement

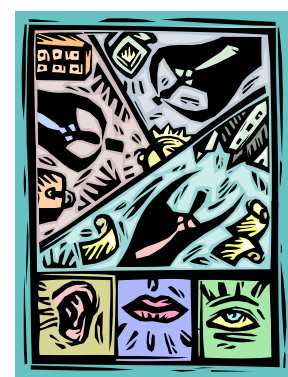

$\checkmark \quad$ If you are having trouble imagining positive outcomes and images, start with imagining an easy skill and work your way up to more difficult skills.

$\checkmark$ Imagine your skills in "real time" - the time it would actually take you to perform them

$\checkmark$ Use imagery in multiple settings - you may use it at home or

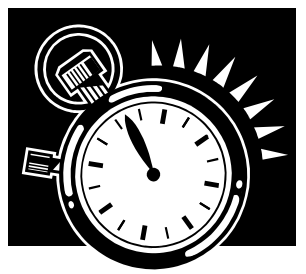
during rehabilitation (e.g., during stim treatment or icing, during cardio) 


\section{Sample Imagery Scripts}

\section{Sample Soothing Imagery Script}

Find a comfortable position and close your eyes. Imagine yourself in a meadow on top of a mountain. It is a warm spring day, feel the sun warming you as you stand in the meadow. The wind is blowing gently. Feel the wind blow through your hair and see the wildflowers sway with the wind. Take a deep breath and smell the wildflowers and the trees. Now walk towards the forest that is a few steps away. Once in the forest you come to small stream. Hear the gurgling of the water as it rushes by. Take off your shoes and wade in the water. Feel the smooth pebbles under your feet and the cool water hitting your ankles. You sit on a nearby rock and breathe in the fresh forest air. Just sit and relax, enjoying the smell of the forest, the sounds of the wind blowing through the trees, and the feeling of the cool water running over your feet.

Which perspective did you use during this imagery? (Circle answer)

Internal External

Rate the vividness and controllability of your images on a scale of 1 (very poor) to 10 (excellent):

Vividness Controllability

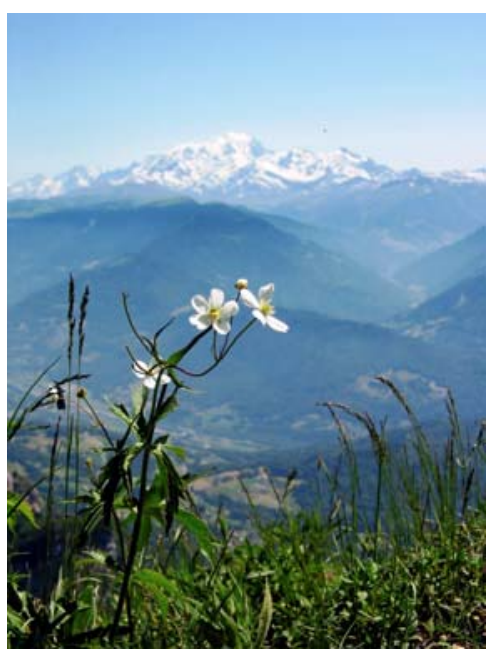




\section{Sample Rehabilitation Imagery Script}

Imagine yourself in the training room. Look around and see all of the training tables and equipment. Take in the familiar smell of the training room. Notice any other athletes and trainers around and hear their chatter. You approach (your trainer) and ask (him/her) what the next exercise is that he wants you to do. He replies (rehab exercise) and tells you to do (\# reps/sets). Go over to the equipment you need. Picture yourself doing (the exercises) perfectly. 1...2 ...3...keep going...Y You can feel the muscles getting stronger with each repetition you do. You may feel some strain, but that's OK. You welcome this sensation because you know it is helping you get better. Finish up your set. When you have finished, you commend yourself for your hard work. Your shoulder feels good and strong and you know that the hard work will pay off.

Which perspective did you use during this imagery? (Circle answer)

Internal

External

Rate the vividness and controllability of your images on a scale of 1 (very poor) to 10 (excellent):

Vividness

Controllability 


\section{Sample Performance Imagery Script}

Imagine yourself in your first wrestling match since your surgery. You feel strong, your shoulder feels stronger than it ever has before. You are on deck and you do your normal routine to prepare for your match. Now it is your turn. You step onto the mat and size up your opponent. You can smell the rubber of the mat mixed with sweat. You feel confident and ready. You can do this. You hear your coach and teammates cheering and yelling for you. The whistle blows and the match begins. See yourself wrestling with your opponent. You hear your coach and teammates yell instructions at you. You are doing well, you can tell that your opponent is faltering. Finally, you see yourself pinning your opponent. You have won! The referee raises your arm high. The feelings of joy and excitement rush over you. Your hard work in the training room and practice has paid off. Take a few moments and enjoy this feeling of accomplishment.

Which perspective did you use during this imagery? (Circle answer)

$$
\text { Internal External }
$$

Rate the vividness and controllability of your images on a scale of 1 (very poor) to 10 (excellent):

Vividness ___ Controllability

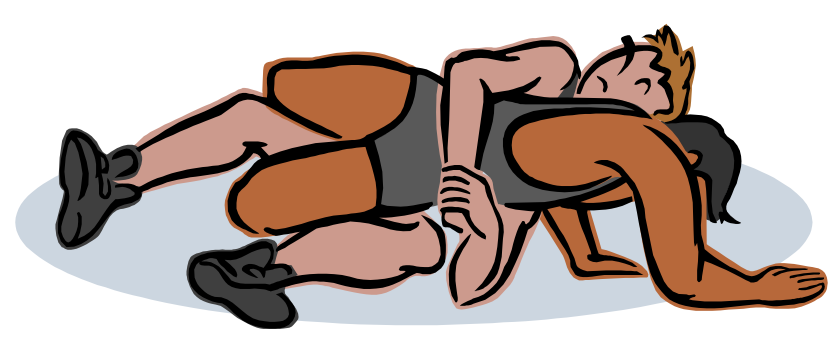




\section{Imagery Log}

Record when you practice imagery and your thoughts about the experience.

\begin{tabular}{|l|c|c|}
\hline DATE & $\begin{array}{c}\text { DESCRIPTION OF IMAGERY } \\
\text { (type, setting, internal/external perspective) }\end{array}$ & $\begin{array}{c}\text { COMMENTS } \\
\text { (what this experience was like, } \\
\text { effectiveness, vividness and controllability) }\end{array}$ \\
\hline & & \\
\hline & & \\
\hline & & \\
\hline & & \\
\hline & & \\
\hline & & \\
\hline & & \\
\hline & & \\
\hline & & \\
\hline & & \\
\hline & & \\
\hline & & \\
\hline & & \\
\hline & & \\
\hline
\end{tabular}




\section{APPENDIX Q:}

Participant Graphs for: Self-efficacy for Return to Sport, Adherence, Attitude, Recovery Time
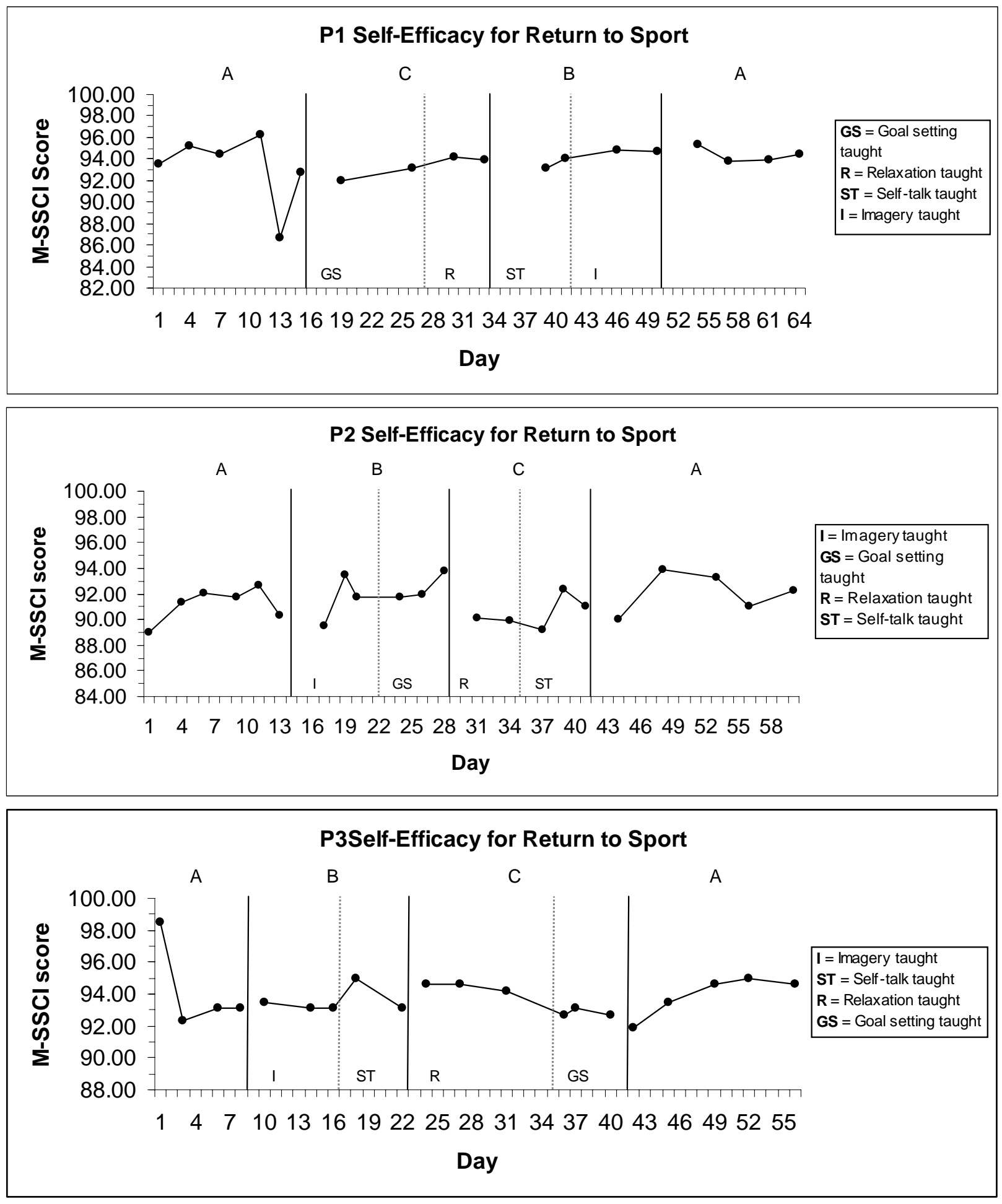

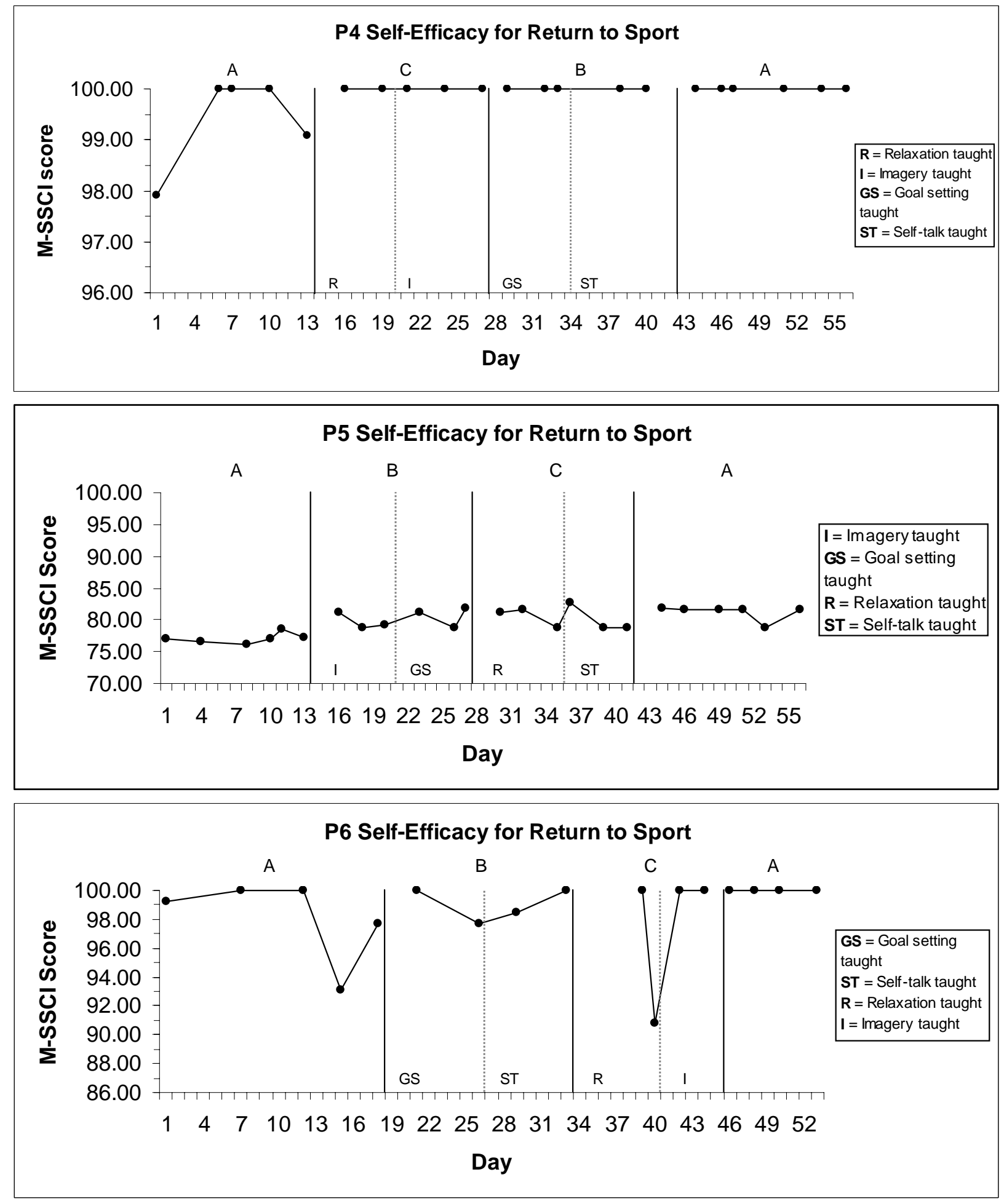

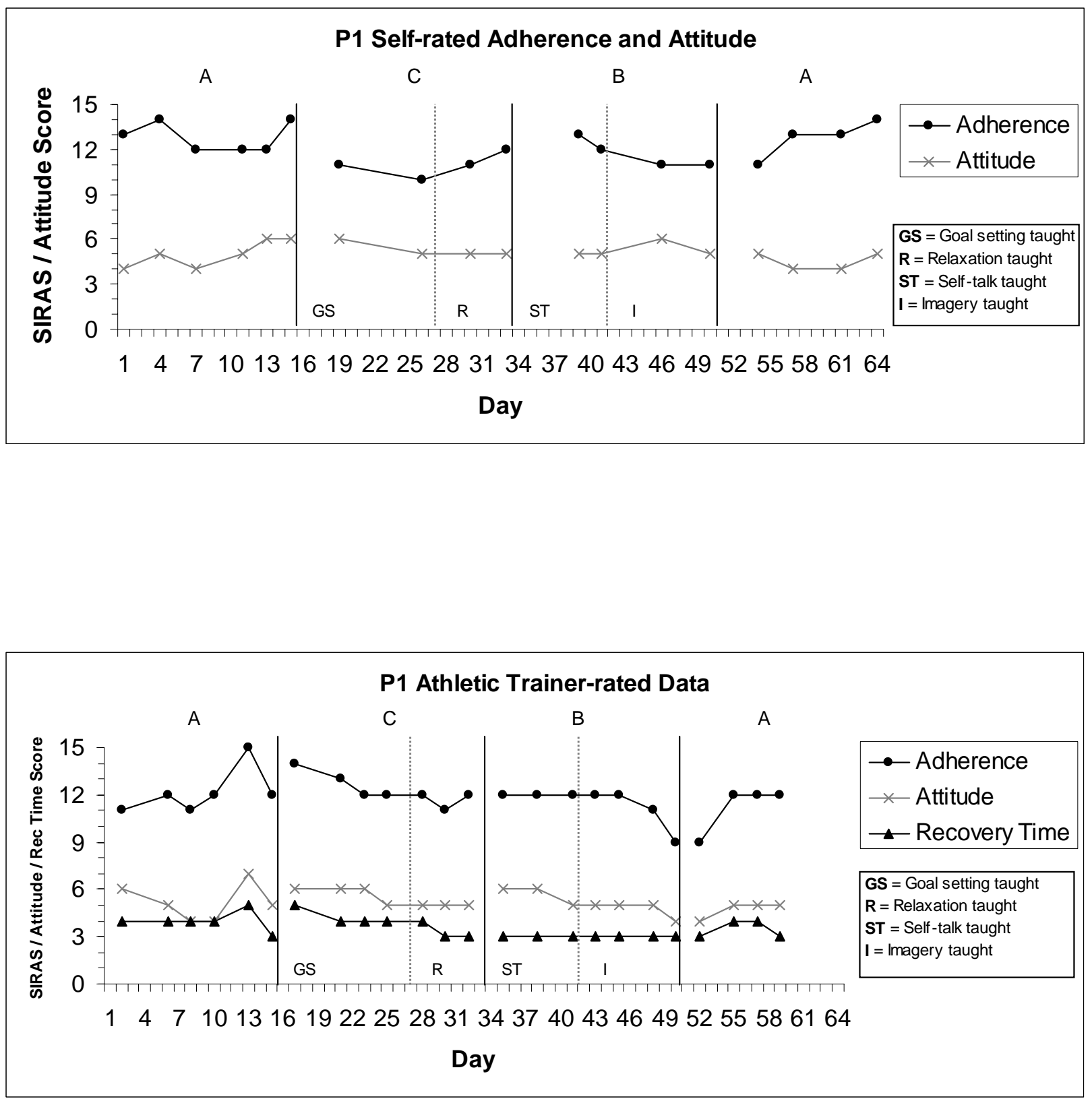

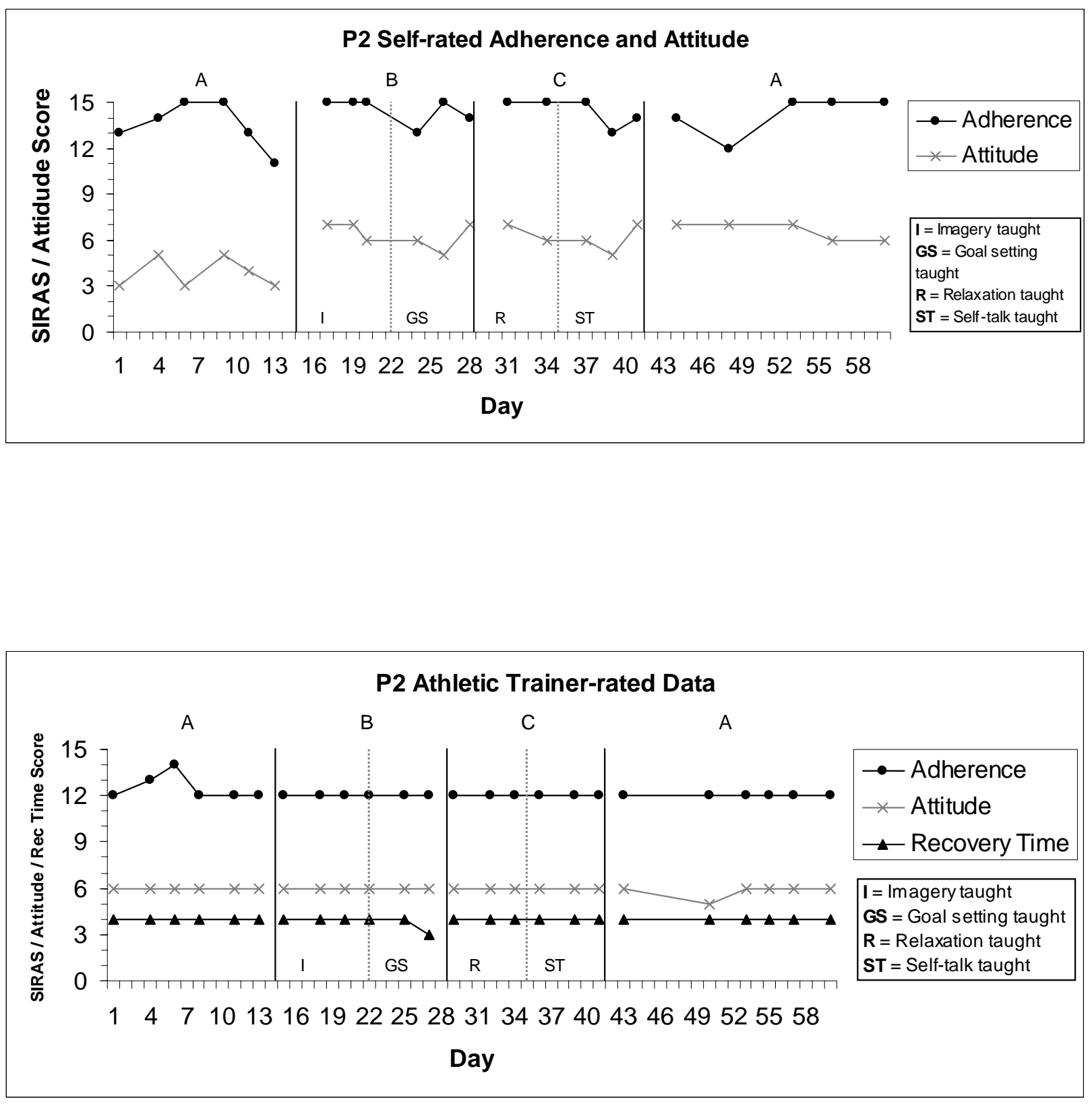

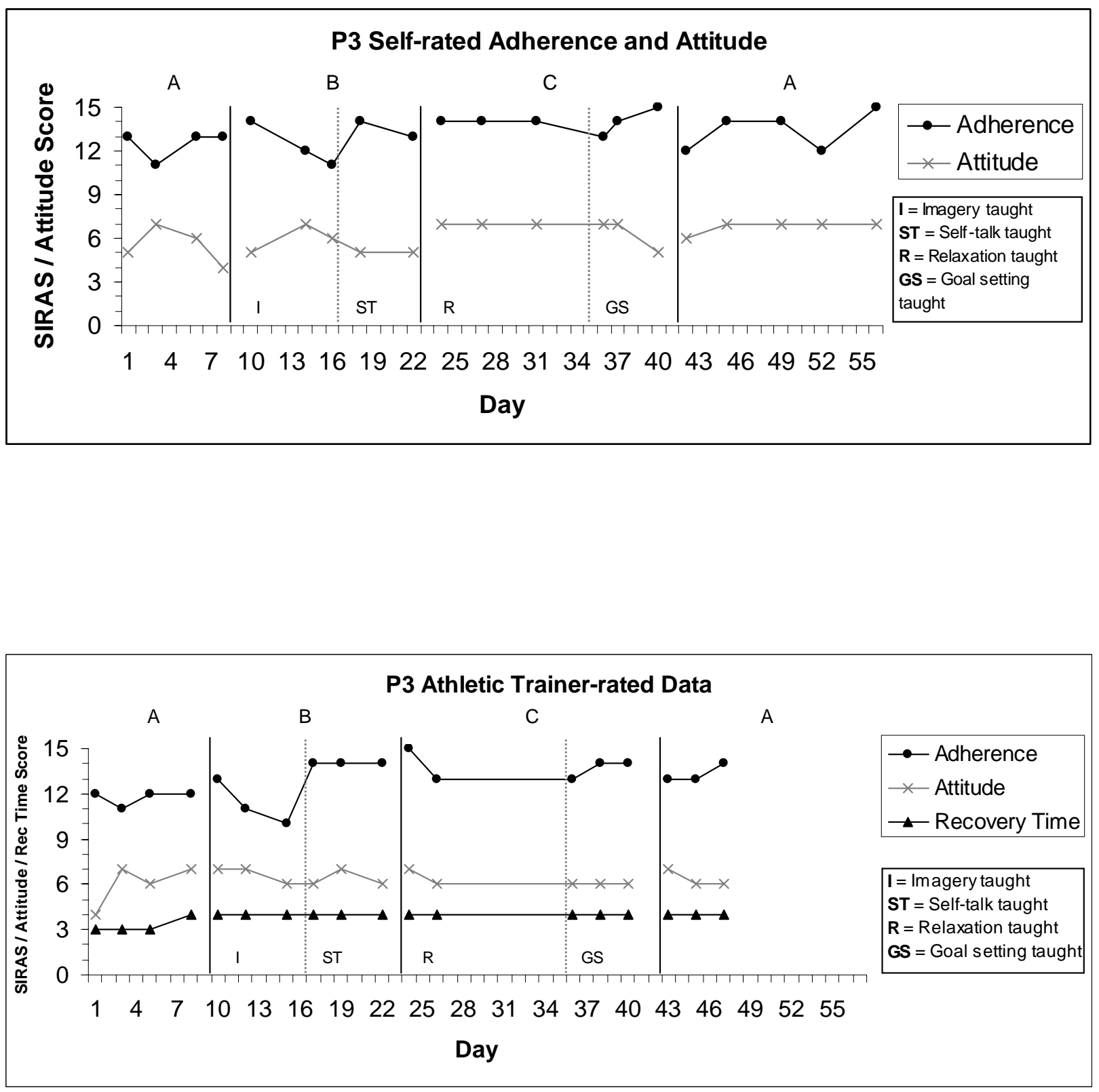

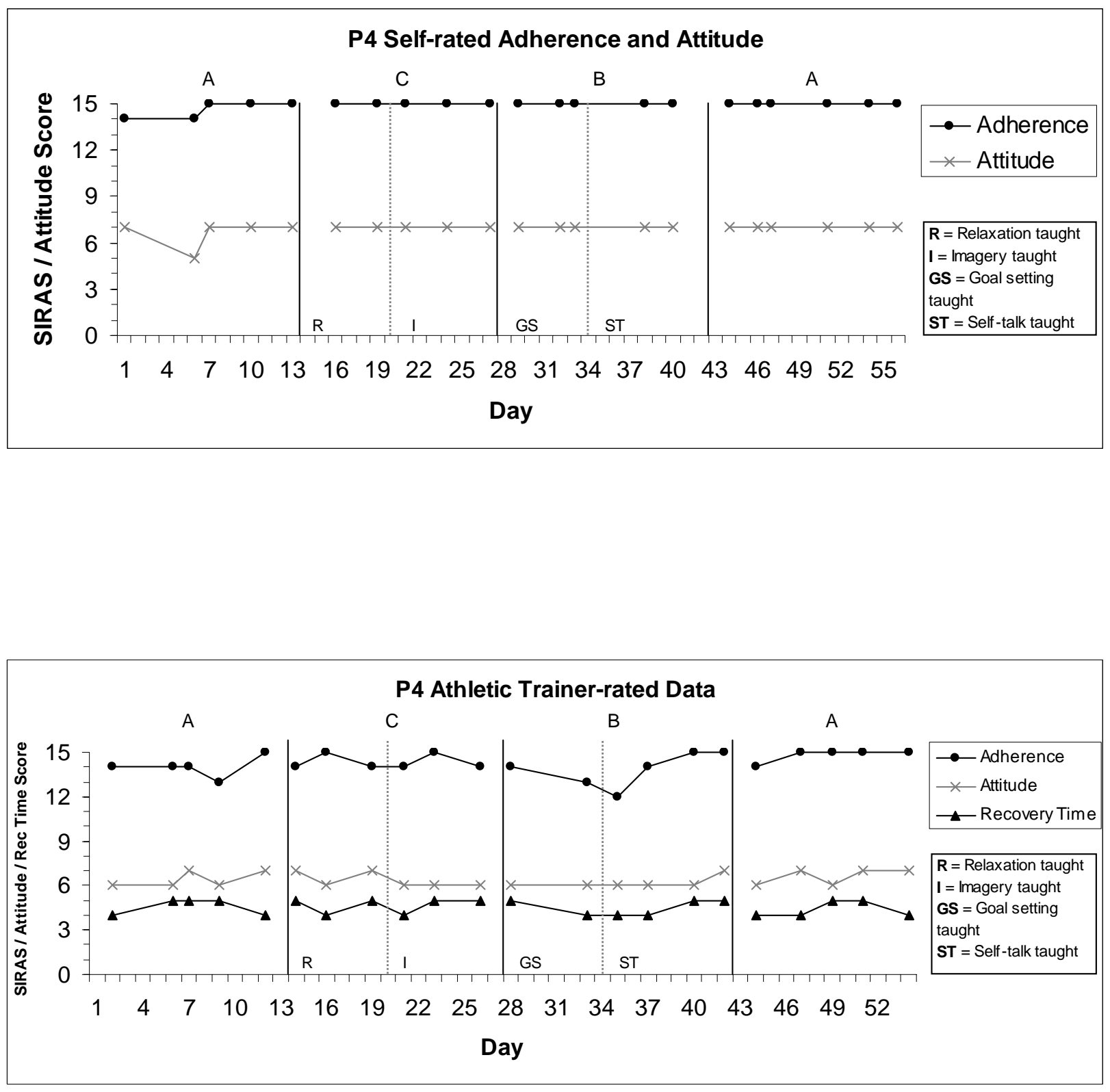

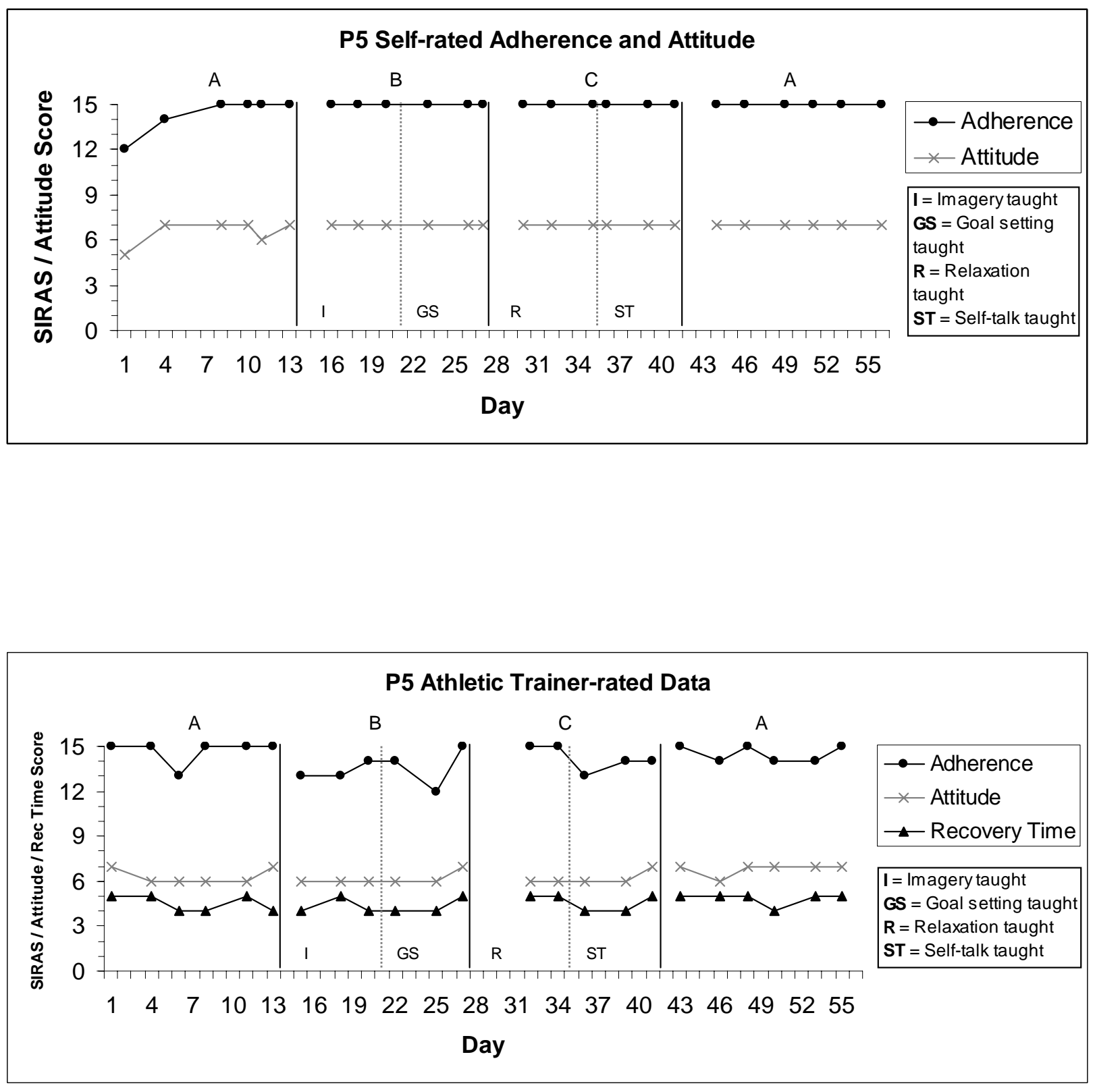

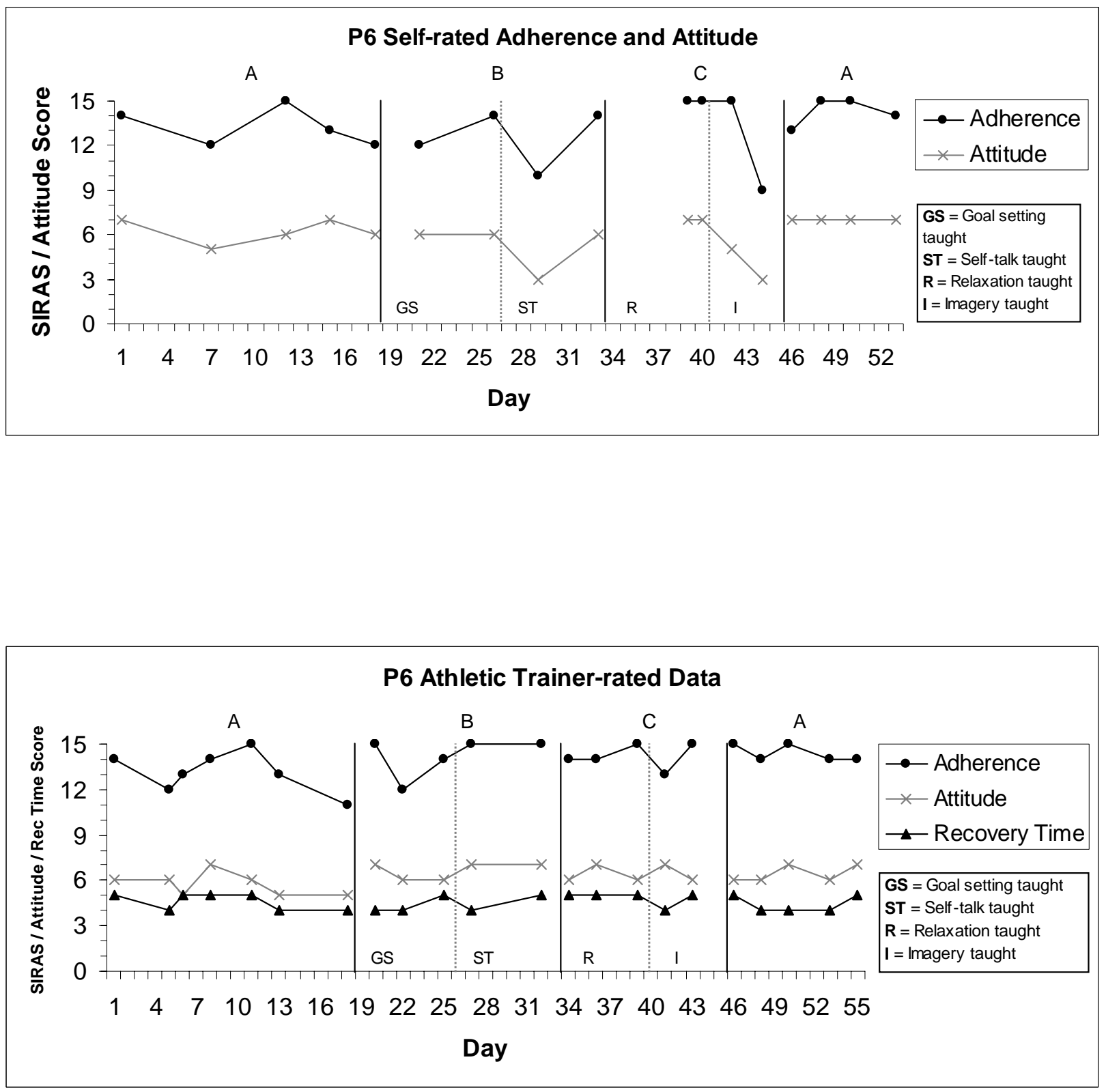